\title{
Nonparametric estimation of blood alcohol concentration from transdermal alcohol measurements using alcohol biosensor devices
}

\author{
By \\ Bryan E. Vader \\ A THESIS SUBMITTED IN PARTIAL FULFILLMENT OF THE \\ REQUIREMENTS FOR THE DEGREE OF \\ MASTERS IN SCIENCE \\ (MATHEMATICS) \\ at the \\ CALIFORNIA STATE UNIVERSITY - CHANNEL ISLANDS
}

2019 
(C) 2019

Bryan E. Vader

\section{ALL RIGHTS RESERVED}


APPROVED FOR THE MATHEMATICS PROGRAM
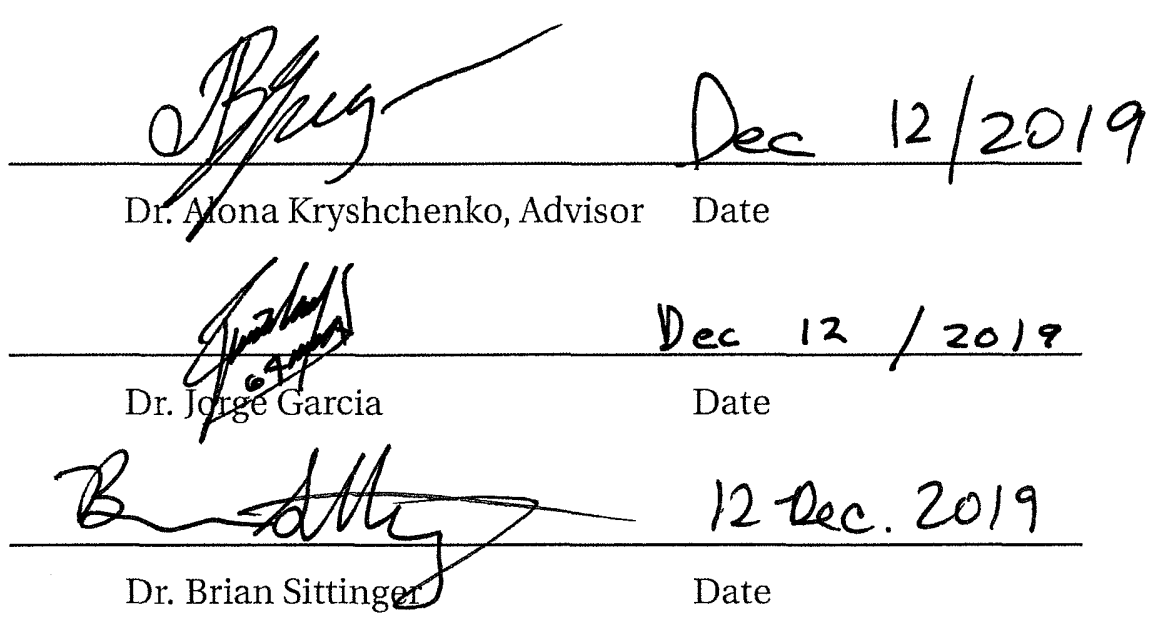

APPROVED FOR THE UNIVERSITY

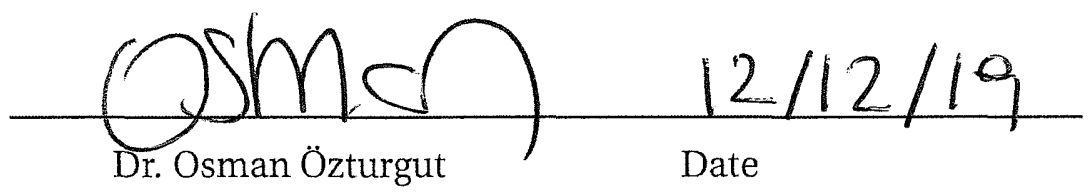




\section{DEDICATION}

This work is dedicated to every extraordinary educator, friend, and family member who saw fit to share their knowledge and patience with me throughout the years. It is also my honor to acknowledge the graduate students in my cohort who made me laugh and challenged my ideas while showing me true friendship. Thank you Matthew Costa, Seungju Lee, Chase Sariaslani, and Mollie Zechlin. Special gratitude is extended to my research advisor Dr. Alona Kryshchenko. Without her guidance, I would be lost. Finally, thanks to Larry Newsome, my late great uncle; stoic and heroic.

It is my honor to recognize the contributions, guidance, and support of Dr. Kryshchenko, Dr. Garcia, Dr. Sittinger, Dr. Grzegorczyk, and the Mathematics Department of California State University Channel Islands. This work was made possible by the research on alcohol biosensor devices conducted by Dr. Melike Sirlanci in her doctoral dissertation at USC.

Camarillo, California

December 16, 2019 


\section{Abstract}

Alcohol biosensor devices have the prospect to positively impact medicine and law enforcement by giving a noninvasive method to acquire continuous alcohol readings. We propose to develop a nonparametric estimation algorithm that estimates the joint mixing distribution of the parameters of a heat equation model via a maximum likelihood method. This model is assumed to estimate the diffusion of alcohol through transdermal layers while taking into account measurement error. These parameters are assumed to be random due to natural irregularity in an individual's body conditions and the variability of population data. This is superior to parametric estimation methods since it can capture unusual fluctuations of a subject's condition as well as environmental factors. This will help to ascertain a precise relation between blood alcohol concentration and transdermal alcohol concentration. 


\section{TABLE OF CONTENTS}

1 Introduction $\quad 5$

1.1 Alcohol Biosensor Devices ................ 6

1.2 Thesis Overview . . . . . . . . . . . . . . . . 13

2 Heat Equation Model $\quad 15$

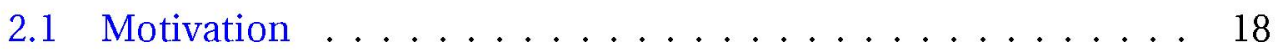

2.2 Heat Equation . . . . . . . . . . . . . . 22

2.2.1 Spacial Discretization $\ldots \ldots \ldots \ldots . \ldots . \ldots 24$

2.2.2 Temporal Discretization . . . . . . . . . . . . 49

2.3 Transdermal Alcohol Concentration ............ 58

2.4 Error in Measurements . . . . . . . . . . . . . . . . 60

3 Estimation of the Joint Mixing Distribution 61

3.1 Maximum Likelihood Estimation . . . . . . . . . . . 63

3.2 Reduction to Finite Support . . . . . . . . . . . . . . 66

4 Data Analysis $\quad 71$

4.1 Simulation of Population Data . . . . . . . . . . . 73 
4.2 Nonparametric Adapted Grid Algorithm . . . . . . . . . . 81

4.2.1 Primal-Dual Interior-Point Method . . . . . . . . . 81

4.2.2 NPAG Algorithm Outline . . . . . . . . . . . . 86

4.3 Consistency and Convergence of the NPAG Algorithm . . . . . 92

4.3 .1 Consistency . . . . . . . . . . . . . 92

4.3.2 Convergence. . . . . . . . . . . . . . 93

5 Conclusions 114

5.1 Future Work . . . . . . . . . . . . . . . . . 117

$\begin{array}{ll}\text { A Background Definitions } & 120\end{array}$

A.1 Probability and Statistics . . . . . . . . . . . . . 120

A.2 Functional Analysis . . . . . . . . . . . . . . . 125

A.3 Convex Analysis . . . . . . . . . . . . . . . . . . 130

A.4 Differential Equations . . . . . . . . . . . . . . . 148

A.5 Numerical Analysis . . . . . . . . . . . . . . . . 152

$\begin{array}{ll}\text { B Algorithms } & 157\end{array}$

B.1 Variables, Constants, and Parameters . . . . . . . . . . 161

B.2 Data Simulation Algorithms $\ldots \ldots \ldots \ldots \ldots \ldots$

B.2.1 Algorithm 1: genpop . . . . . . . . . . . . . . 166 
B.2.2 Algorithm 2: discU . . . . . . . . . . . . . . 168

B.2.3 Algorithm 3: discUerror . . . . . . . . . . . . . 172

B.3 Maximum Likelihood Estimator Algorithms . . . . . . . . 173

B.3.1 Algorithm 4: Nonparametric Adaptive Grid Algorithm 173

B.3.2 Algorithm 5: $\Psi$ : Probability Matrix of Grid Supports . 176

B.3.3 Algorithm 6: Probability of $Y_{i}$ Given the $\xi_{j}$ Support Point 177

B.3.4 Algorithm 7: Condense Grid of Support Points . . . . . 178

B.3.5 Algorithm 8: Expand Grid of Support Points . . . . . 179

List of Figures

List of Algorithms

Bibliography
181

185

186 


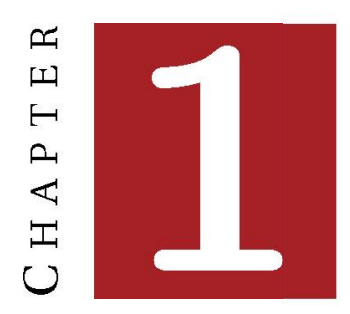

\section{INTRODUCTION}

There is ample modern day interest in transdermal alcohol concentration (TAC), that is, the alcohol concentration in vapor form above the epidermis. We propose that there is a significant statistical relationship between transdermal alcohol concentration and blood alcohol concentration measurements. To begin, we give the reader some history relating to this research. Afterwards, a review of currently available alcohol biosensor devices is provided with notes of current engineering challenges that face the biotechnical sector. The last section provides a brief overview of this thesis to emphasize the primary goalposts of the paper. 


\subsection{Alcohol Biosensor Devices}

Alcohol consumption is an intrinsic part of modern society. As such, there arose a need to regulate alcohol consumption by those that drink irresponsibly. According to Hawthorne and Wojcik [6] in a 2006 article, the interest in transdermal alcohol concentration dates back to 1936 . In that early research Nyman and Palmlöv [15] attempted to estimate what percentage of alcohol consumed ultimately diffuses through the skin. They obtained an estimate of one percent. The next advancements came between the 1960s and the 1980s. Researchers grew interested in the role of sweat glands excreting drugs and alcohol. A sweat patch used to measure alcohol was finally developed in 1984, and the very promising experiment results including a statistically significant connection between alcohol concentration in sweat and blood alcohol concentration (BAC). The fact that they found no false-positives surely spurred the technology forward.

The next obvious question posed is whether transdermal ethyl alcohol excretions from the skin would also show strong correlation to BAC measurements. Late in the 1980s, a lab at Indiana School of Medicine discovered that although TAC did also show a correlation to BAC, the pharmacokinetic parameters were different from breath alcohol concentration (BrAC) or BAC. In the 
same time, the University of Toronto verified that not only did there appear to be a strong correlation, but the researchers could also use the vapors collected at the skin to alert a device to the presence of alcohol. These new wearable devices faced a major issue of 45 minute measurement delays for the human body to metabolize alcohol and begin to provide the initial TAC readings. However, the University of Toronto did show that the TAC curve, which was now available since the measurements occur every more frequently, matched the BAC curve in shape.

Research to develop a device to monitor and prevent further alcohol abuse by individuals began in the 1990s. The most widely used device, SCRAM CAM (see Figure 1.1), commenced design plans in 1991, and it further corroborated the correlation between TAC and BAC, or TAC and BrAC.

Another wrist-worn device, the WrisTAS, was simultaneously produced during this time period and reported more of the same results (see Figure 1.2). A 2013 article by Leffingwell et al [11], in the journal ALCOHOLISM: CLINICAL AND EXPERIMENTAL RESEARCH, provided some detail of the technology present in SCRAM CAM. Some units contain a GPS tracker for law enforcement with location data, since most users are court ordered law offenders. There is an electrochemical alcohol sensor that stores TAC measurements in 


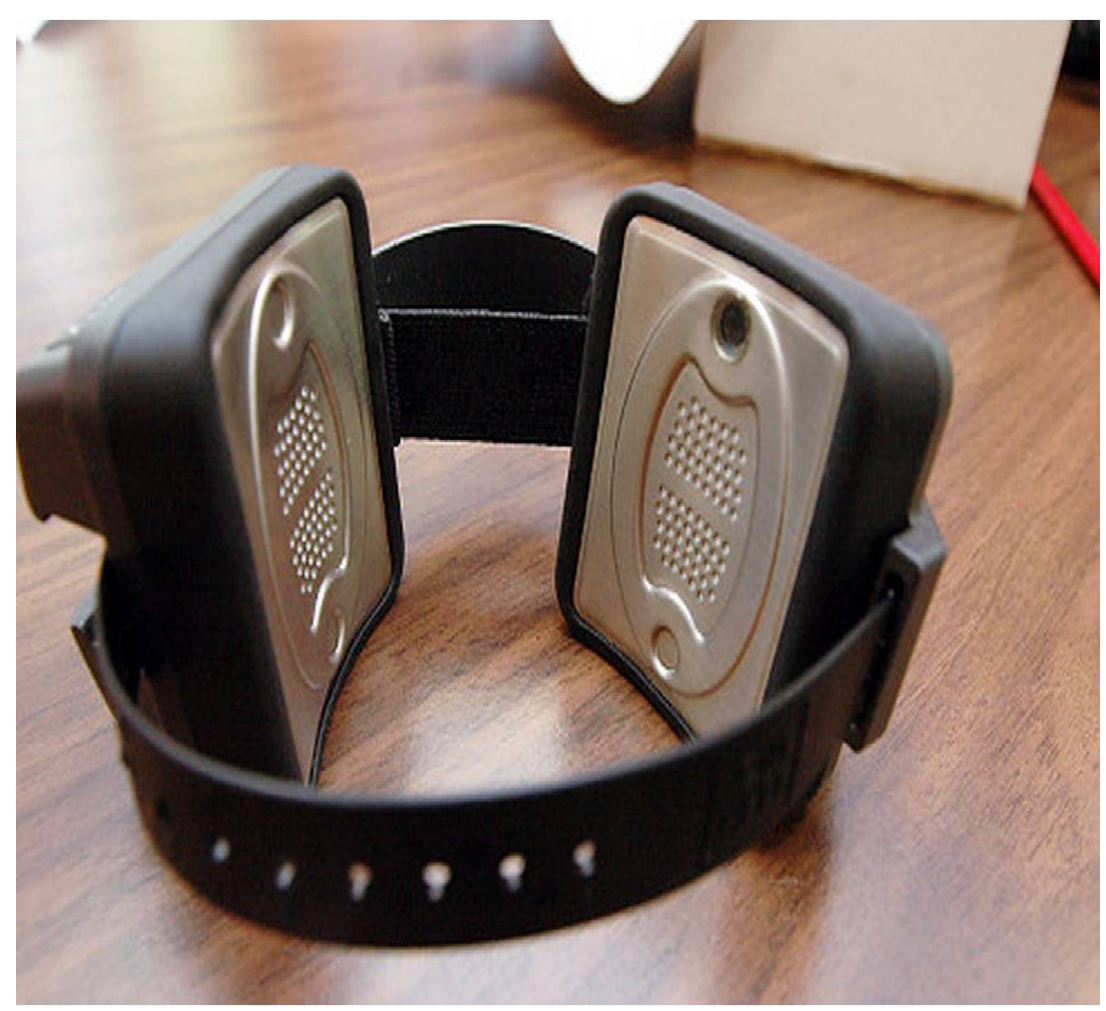

Figure 1.1: SCRAM CAM( Alcohol Biosensor Ankle Device Accessed from www.nydailynews.com

30 minute increments, a sensor to take skin temperature, and a sensor to detect whether there is skin contact. The last two sensors are important to courts when attempting to ascertain whether an individual has tampered with a device.

In 2003, SCRAM launched their continuous alcohol management monitoring system along with their first tested public use-ready ankle alcohol biosensor device. That year they started work with Orange County, CA to track court 


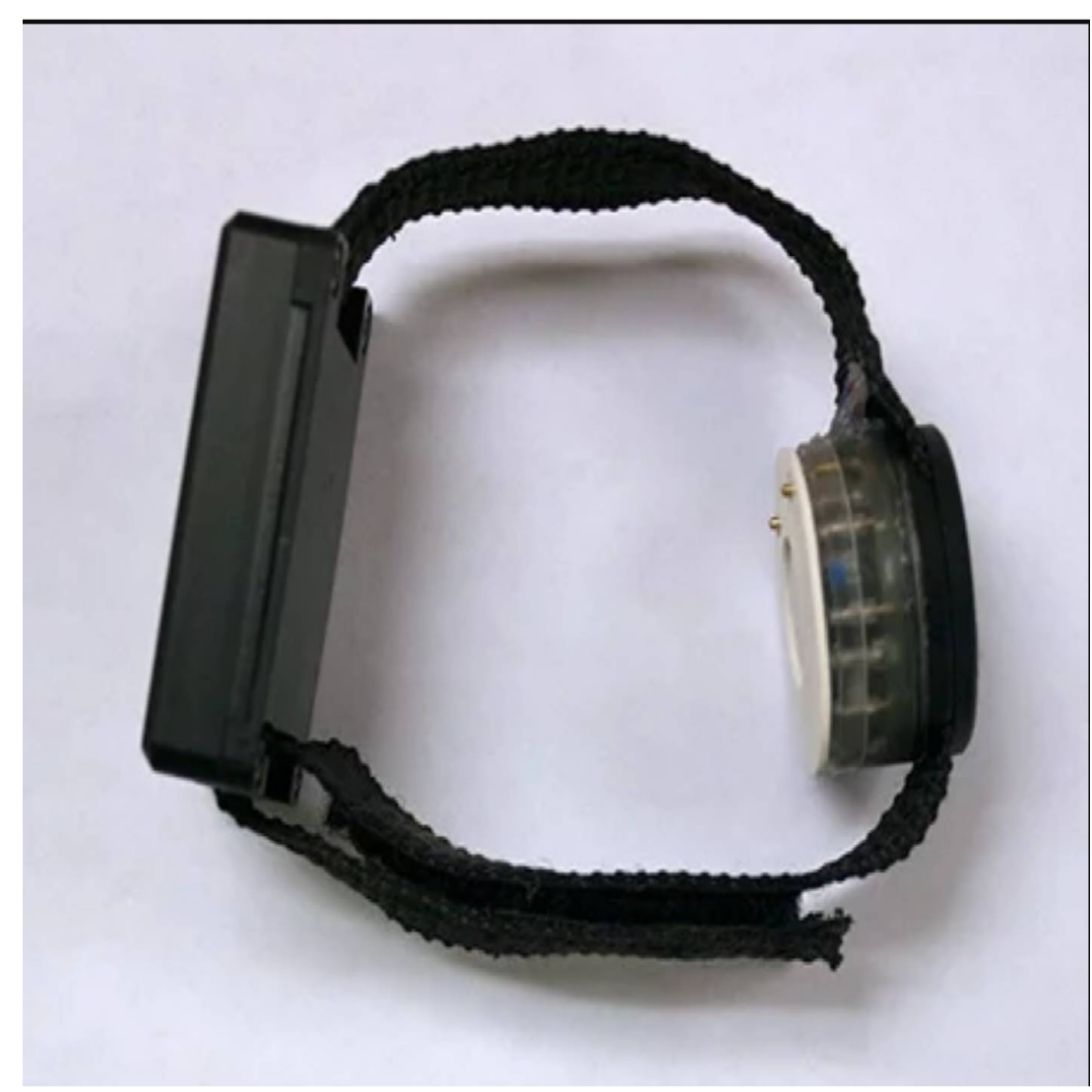

Figure 1.2: WrisTAS7® Alcohol Biosensor Wrist Device Accessed from www.ginerinc.com

parolees. As of 2019, they now monitor millions of TAC readings across 49 states and they are the leading alcohol biosensor in the world. Each year, they continue to develop new monitoring systems to aid law enforcement while ensuring that data systems provide easy access to records for an individual's query.

Even though the SCRAM CAM and WrisTAS devices are both successfully 
used widely to monitor an individual's sobriety, they have technological limitations such as the aforementioned 45 minute lag in readings and the methods used to store data. In particular, the SCRAM CAM requires a wireless station in the home, but it can store up to two weeks of readings if the user is away. The WrisTAS must have the measurement sample downloaded to a computer. Both devices only take a discrete reading every 30 minutes which may or may not be sensitive enough for a given analysis purpose. Finally, neither device can survive being submerged in water.

To address these issues, the National Institute on Alcohol Abuse and Alcoholism, a government funded entity, called for the development of new devices through a competition in 2016 with a $\$ 200 \mathrm{k}$ first prize and $\$ 100 \mathrm{k}$ second prize. Competition rules stated that these new devices should capitalize on modern technology like Bluetooth and cutting edge biosensors to expand the use of alcohol biosensor devices into areas others than just sobriety management. In particular, teams should design and engineer the biosensors as wearable bands that can be attached to watches or some similar discreet form factor. The winner of the competition was BACtrack Skyn (see Figure 1.3), a company which is well known for their alcohol breathalyzers.

According to the NIAAA website, BACtrack Skyn was chosen, because it 


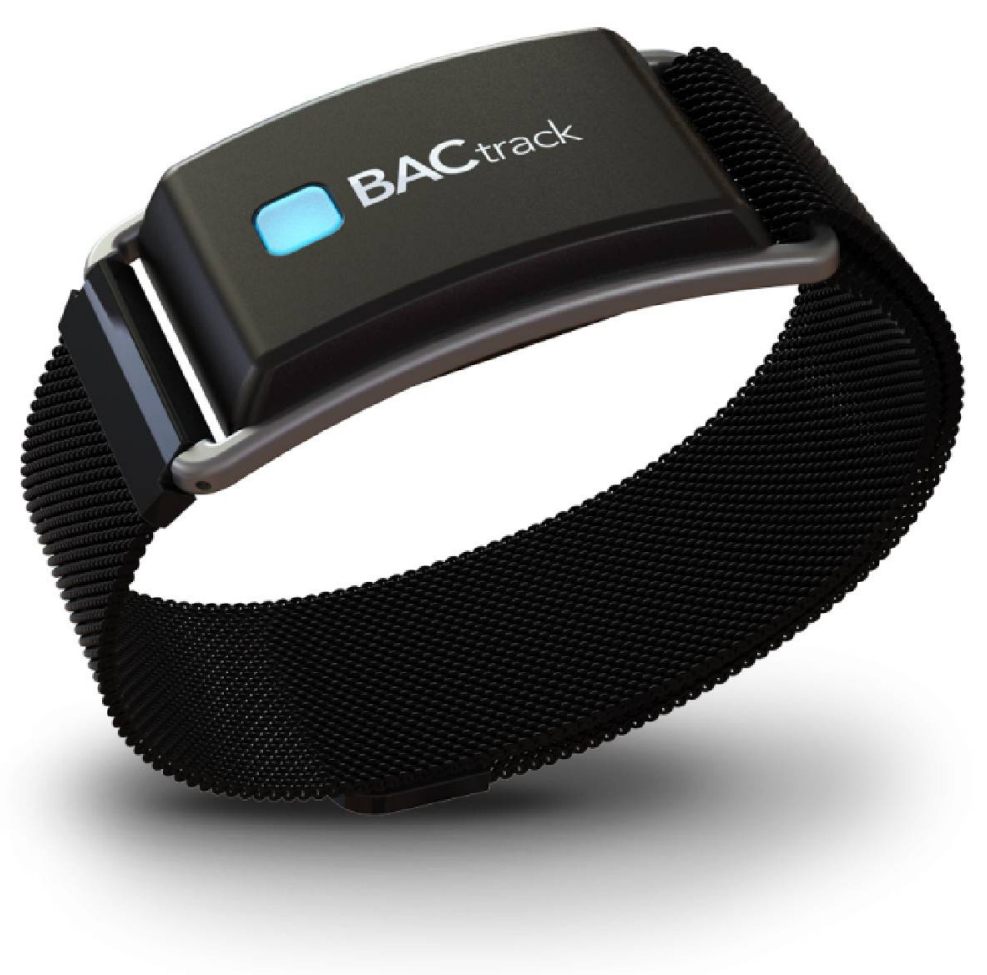

Figure 1.3: BACtrack Skyn Alcohol Biosensor Wrist Device Accessed from https://skyn.bactrack.com/

appears as a light fitness wristband and it provides true continuous and noninvasive monitoring of a user's BAC via TAC measurements. The device detects alcohol through a fuel cell technology similar to that used in devices by law enforcement for roadside alcohol testing. As a modern benefit, it connects via Bluetooth to a smartphone to store data.

It will be interesting to see if this new device replaces SCRAM CAM or Wris- 
TAS as the prevalent technology, or if this sparks an innovation war to the benefit of the consumer. This updated device may be what is needed to bring the alcohol biosensors into new industries such as medicine. BACtrack Skyn still needs an appropriate testing phase before public release. 


\subsection{Thesis Overview}

The goal of this research aims to develop a nonparametric estimation algorithm that calculates the joint mixing distribution of the parameters of a heat equation model via a maximum likelihood method which also accounts for measurement error. It will ascertain a precise relation between blood alcohol concentration and transdermal alcohol concentration.

To test the hypothesis that there is a significant statistical relationship between TAC and BAC measurements, we develop a model for data simulation in Chapter 2. Each individual in the population will have distinctive parameters that relegate diffusion through dermal tissue. In Chapter 3, we present the theory to show that the unknown density mixing distributions for the diffusion parameters can be estimated using a nonparametric maximum likelihood from a simulated sample consistent with human biology. In Chapter 4, we give an algorithm to compute the problem presented in Chapter 3. Figure 1.4 below shows the major steps of the procedure towards estimation.

Figure 1.4: Thesis Overview Diagram

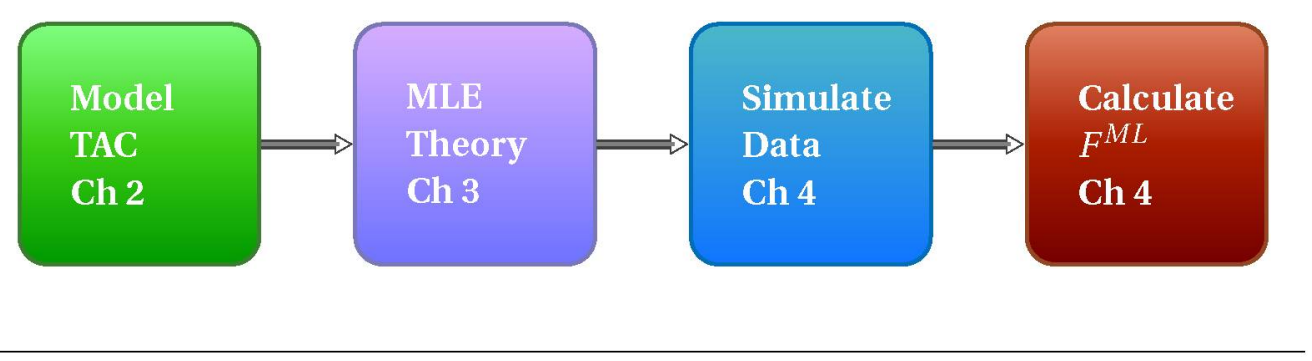


Note that this project is not concerned with a specific alcohol biosensor device, because the data in this research is simulated. To achieve a theoretically sound maximum likelihood estimate for the joint mixing distribution of the parameters of diffusivity, a method called the Nonparametric Adaptive Grid (NPAG) algorithm is employed. It is the natural progression of a Nonparametric Expectation-Maximization (EM) algorithm developed by Dr. Alan Schumitzky in the 1980s. An adaptive grid method was added by Dr. Robert Leary in the 1990s where the grid of support points is adjusted to coalesce around points with highest probability. Combining this with the Primal-Dual Interior-Point (PDIP) methods on the adapted grid yields NPAG. 


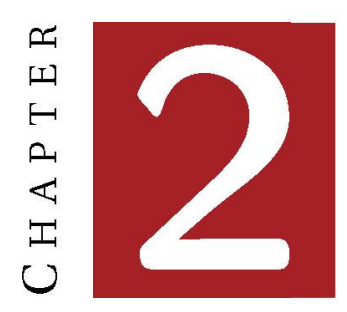

\section{Heat Equation Model}

A human subject undergoes a complex chain of metabolic reactions and waste processing after the oral consumption of alcohol. The complete set of pathways involves several compartments of the body. We are concerned with only the diffusion of alcohol particles from a region of high alcohol concentration the blood stream through the dermal layer to a region of low alcohol concentration in the atmosphere; see Figure 2.1.

It is reasonable to assume that the heat equation, a partial differential equation which is a well-known case of the diffusion equation in physics, sufficiently models this biological process as well. This is due to the similarities of how heat and alcohol particles both diffuse through a solid medium over a 
period of time. 


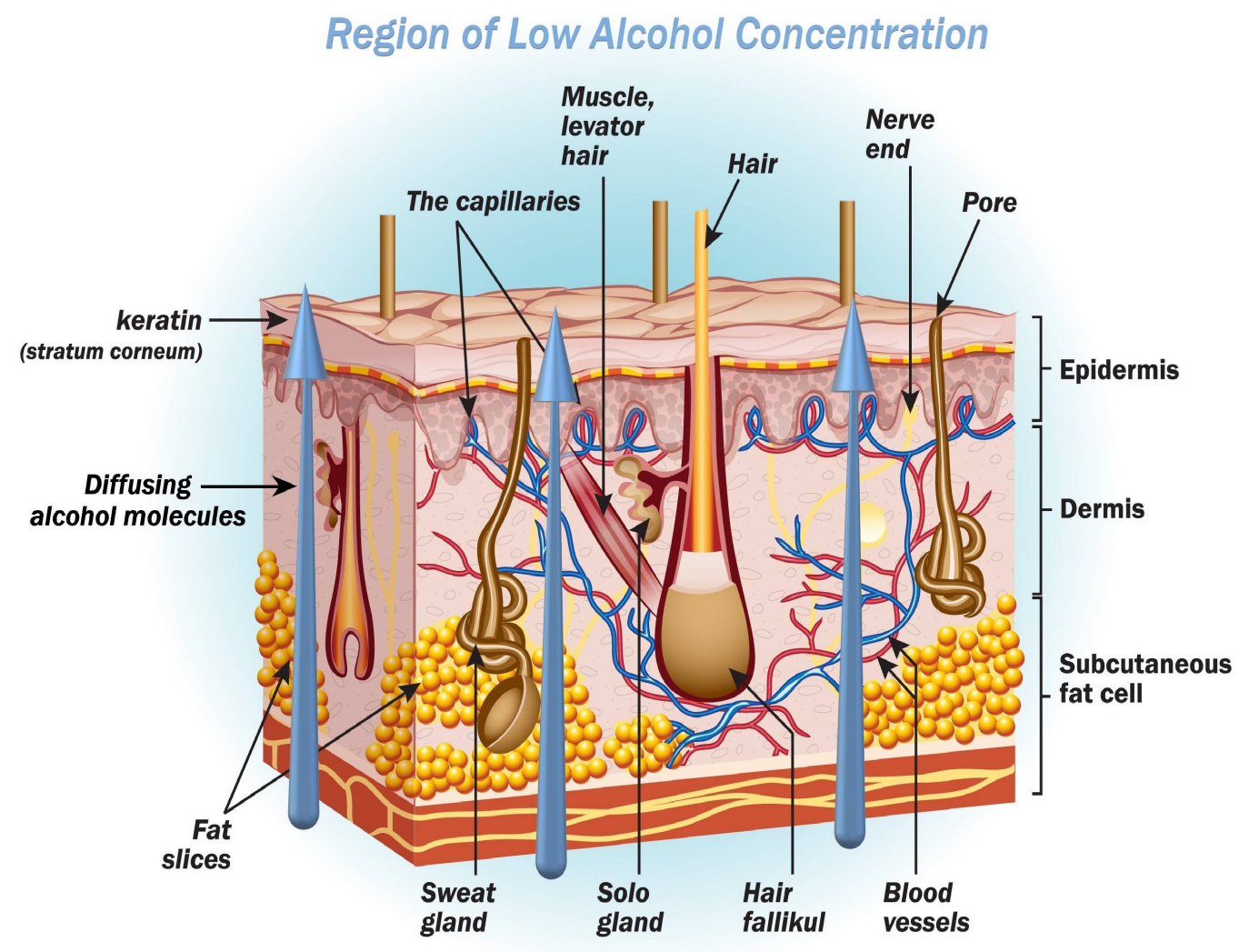

Region of High Alcohol Concentration

Figure 2.1: Alcohol Diffusing Through the Dermal Layer 


\subsection{Motivation}

The physical process of heat transfer goes through a solid medium from a region of high temperature to a region of low temperature over a period of time, such as the rod as shown in Figure 2.2, is modeled by the partial differential

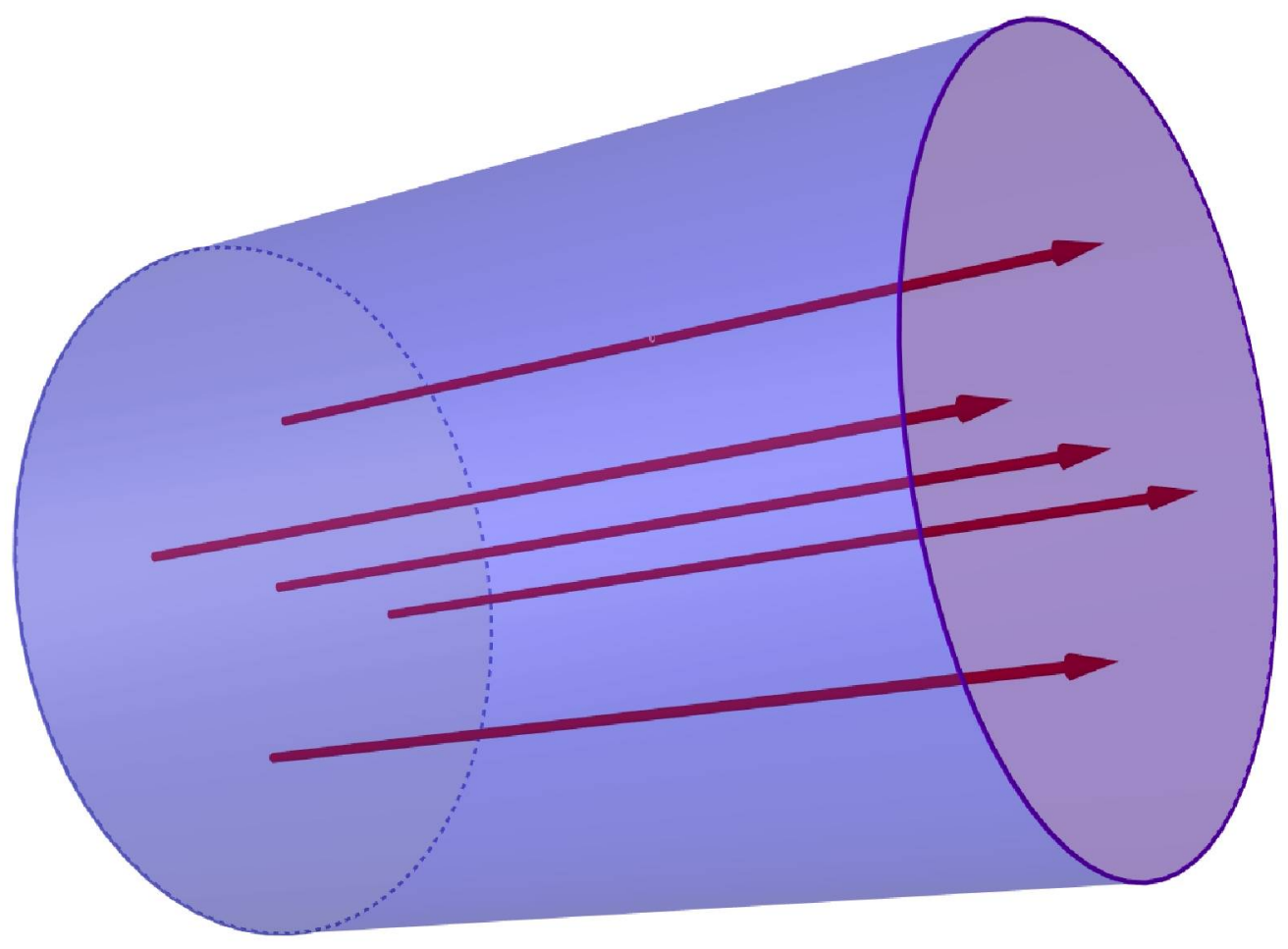

Figure 2.2: Heat Transfer Through a Rod

equation known as the heat equation (see Definition A.4.1).

Let $u(x, y, z, t)$ be the scalar function for alcohol concentration. Let $c$ be the specific heat, which is the amount of heat energy input required to raise 
the temperature by one unit in one unit of mass of the medium; this is measured in joules per kelvin per kilogram, $J /(K \cdot k g)$. Observe that the regulated body temperature of the individual should remain effectively constant, because the alcohol diffusion process should not measurably affect it. Hence, we assume that $c$ is constant as well. Let $\rho$ be the density of the dermal tissue; this is measured in kilograms per cubic meter, $\mathrm{kg} / \mathrm{m}^{3}$. Since the small region of the dermal layer, where the alcohol biosensor contacts the skin, is assumed to be uniformly structured, we also assume that $\rho$ is constant. Let $\kappa$ be the heat conductivity (particle conductivity in this context); this is measured in watts per meter-kelvin, $W /(m \cdot K)$. Given that the three physical properties $c$, $\rho, \kappa$ are constant, this is a special case of the diffusion equation where the rate of heat transfer (with respect to time) is reliant upon the diffusivity constant for the specific medium. Hence, we define $q_{1}=\frac{\kappa}{c \rho}$, as in Definition A.4.1, to be this constant of diffusivity.

We now show how the biological aspects of this dermal diffusion problem fit to the heat transfer theory. Observe that the mathematical analysis of alcohol diffusion through the dermal layer also appears to suit the heat transfer model formulation. First, we calculate the flux of alcohol molecules through 
a surface. Let

$$
U=\nabla u=\left\langle\frac{\partial u}{\partial x}, \frac{\partial u}{\partial y}, \frac{\partial u}{\partial z}\right\rangle
$$

be the vector field of the scalar function $u$ in three-dimensional space with $t$ being held constant (but arbitrary). Let $S(V)$ be the locally flat skin surface having the dermal volume $V$. By the divergence theorem, we can express the flux of the vector field $U$ as

$$
\Phi_{U}(t)=\iiint_{V} \nabla \cdot U \mathrm{~d} V
$$

In this problem, the direction of the diffusing particles is assumed to be normal to the planar skin surface, because the alcohol molecules will follow the most direct pathway to a region of low alcohol concentration. In later research, we intend to additionally consider small irregularities in the path through the dermal layer. Then, since there is zero change in alcohol concentration in the $x$ or $y$ directions the vector field reduces to

$$
U=\left\langle 0,0, \frac{\partial u}{\partial z}\right\rangle
$$


Applying Equation (1.3) to the flux equation above gives us

$$
\begin{aligned}
\Phi_{U}(t) & =\iiint_{V} \nabla \cdot U \mathrm{~d} V \\
& =\iiint_{V} \nabla \cdot\left\langle 0,0, \frac{\partial u}{\partial z}\right\rangle \mathrm{d} V \\
& =\iiint_{V} \frac{\partial^{2} u}{\partial z^{2}} \mathrm{~d} V
\end{aligned}
$$

Hence, the mathematical heat diffusion model used in the following section is simplified to ignore the $x y$-planes parallel to the skin and to only consider the dermal depth spacial dimension. Please see Definition A.4.1 in Appendix A for more information on the general formulation of the heat equation. 


\subsection{Heat Equation}

This section creates a discretized solution to the partial differential equation heat equation model while taking into consideration appropriate boundary conditions befitting the biology. Let $V=\mathbb{R}^{4}$ be a Hilbert space and $V^{*}$ be the dual space of $V$. Let $\eta \in \mathbb{R}$ denote the depth of skin measured in centimeters, where $\eta=0$ is the surface of the dermal layer and $\eta=L$ is the bottom of the dermal tissue. Let $t \in \mathbb{R}$ be the time in seconds at which the measurement is taken. Let $q=\left\langle q_{1}, q_{2}\right\rangle \in \mathbb{R}^{2}$ be the parameters where $q_{1}$ measured in $\mathrm{cm}^{2} / \mathrm{sec}$, and $q_{2}$ measured in TACunits·BrACunits/BACunits, are constants of diffusivity. Define $u(\eta, t ; q) \in V$ as the concentration of alcohol measured in

moles $/ \mathrm{cm}^{2}$ at time $t$ and depth $\eta$ with parameters $q$. Define $\hat{\eta}=\frac{\eta}{L}$ to be the normalized depth. For the duration of this paper, $\hat{\eta}$ will be referred to as $\eta$ and all equations dependent on $\eta$ will be assumed to be appropriately parameterized to be in terms of $\hat{\eta}$. See Figure 2.3 for an image showing the orientation of the dermis with respect to an axis.

We now pose appropriate boundary conditions. We assume that the individual is initially sober. Hence, $u(\eta, 0)=0$ for $0<\eta<1$; this is recorded as Equation (2.8) in the system below. Next, consider the alcohol concentration at an arbitrary positive time measured at skin level. This transdermal 


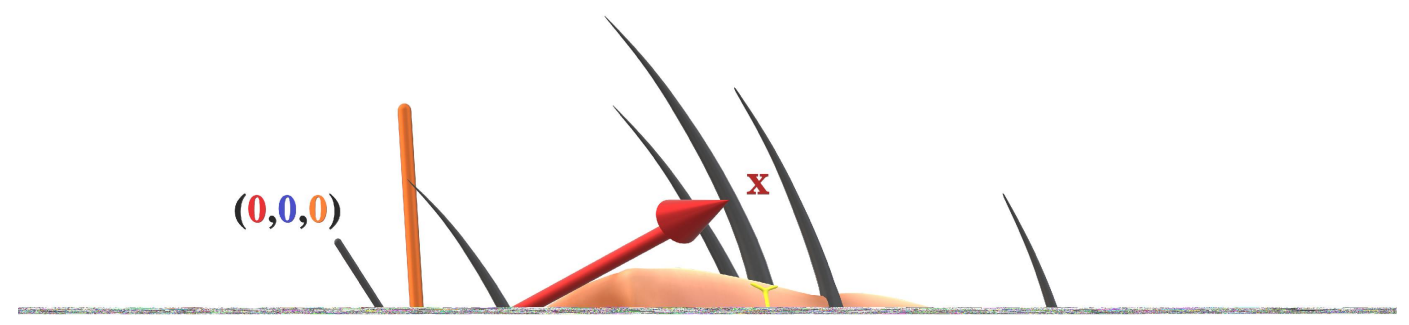

Figure 2.3: Dermal Layer Orientation With Respect to an Axis

measurement is assumed to be directly proportional to the change of alcohol concentration as the molecules pass over and out of the skin. Then, $u(0, t)=$ $q_{1} \frac{\partial u}{\partial \eta}(0, t)$ for $t>0$; this is recorded as Equation (2.9) in the system below. Finally, we assume that the input of alcohol orally ingested by the individual is directly proportional to the rate of alcohol passing from the blood stream into the lowest level of the dermal tissue. The equation is $\frac{q_{1}}{q_{2}} \frac{\partial u}{\partial \eta}(1, t)=\alpha(t)$ for $t>0$; 
this is recorded as Equation (2.10) in the system below.

The following system summarizes the heat equation with boundary conditions in weak formulation (see Definition A.4.4)

$$
\begin{array}{rlrl}
\frac{\partial u}{\partial t}(\eta, t) & =q_{1} \frac{\partial^{2} u}{\partial \eta^{2}}(\eta, t), & & t>0,0<\eta<1, \\
u(\eta, 0) & =0, & & 0<\eta<1, \\
u(0, t) & =q_{1} \frac{\partial u}{\partial \eta}(0, t), & t>0, \\
\frac{q_{1}}{q_{2}} \frac{\partial u}{\partial \eta}(1, t) & =\alpha(t), & t>0 .
\end{array}
$$

Please note that the analysis and calculations of the following two sections largely benefits from the doctoral dissertation of Dr. Sirlanci [23]. Small variations may exist due to the differing methods of estimation in each paper.

\subsubsection{Spacial Discretization}

In this section, we discretize the heat equation spatially. We accomplish this by using the finite element method (see Definition A.5.7), which is a special case of the classical Galerkin method (see Definition A.5.6) that requires that the test functions are piecewise polynomials. In the text "Numerical Solution of Partial Differential Equations by the Finite Element Method" [8] Johnnson 
explains that to solve a given differential or integral equation approximately using the finite element method, one has to complete the following steps

(i) variational (weak) formulation of the given problem,

(ii) discretization using FEM: construction of the finite-dimensional space $V_{h}$,

(iii) solution of the discrete problem,

(iv) implementation of the method on a computer: programming.

We addressed item (i) regarding weak formulation in the previous section, and complete items (ii) and (iii) in this section. The algorithm for item (iv) is detailed in Appendix B and implemented in Chapter 4.

To begin, take an inner product on both sides of the heat equation in Equation (2.7) with a test function $\varphi(\eta)$.

$$
\begin{aligned}
\frac{\partial u}{\partial t}(\eta, t) & =q_{1} \frac{\partial^{2} u}{\partial \eta^{2}}(\eta, t) \\
\left\langle\frac{\partial u}{\partial t}(\eta, t), \varphi(\eta)\right\rangle_{V^{*}} & =\left\langle q_{1} \frac{\partial^{2} u}{\partial \eta^{2}}(\eta, t), \varphi(\eta)\right\rangle_{V^{*}}
\end{aligned}
$$

Next, we discretize $\eta$ by uniformly partitioning $[0,1]$ into $N$ parts. Define $V_{h}$ to be the finite-dimensional subspace of $V$ where each piecewise-defined function in $V_{h}$ agrees with boundary conditions of continuous functions in $V$. This satisfies item (ii) of the finite element method. 
To accomplish the task of evaluating Equation (2.11), we choose the test function $\varphi(\eta)$ to be an appropriate minimal order of B-spline (see Definition A.5.3). These spline functions have minimal support in order to simplify the algorithmic complexity and thus minimize computation time while still providing a strong enough numerical approximation to a curve. Define $\hat{\varphi}(\eta) \in V$ as an $(N+1) \times 1$ vector of order-two B-spline equations evaluated at $\eta$. Note these are often called "linear B-splines" or "tent functions" due to their shape. This is the minimal feasible order, because forthcoming calculations will require the partial derivatives of these spline functions to be well-defined. $(\mathrm{Ob}-$ serve that the derivative of a line is its slope, while the derivative of an indicator function is everywhere zero.) See Figure 2.4 for an example how linear splines fit to a curve.

The $i^{t h}$ entry of $\hat{\varphi}(\eta)$ is $\varphi_{i}(\eta)$ which is the $i^{t h}$ basis spline. Define $\varphi_{i}(\eta)$, for $i=1, \ldots, N-1$ as

$$
\varphi_{i}(\eta)= \begin{cases}N \eta-i+1 & \text { if } \frac{i-1}{N} \leq \eta<\frac{i}{N} \\ -N \eta+i+1 & \text { if } \frac{i}{N} \leq \eta<\frac{i+1}{N} \\ 0 & \text { elsewhere. }\end{cases}
$$

The $1^{\text {st }}$ linear B-spline is unique from the others, due to how it only has the decreasing line on the right side of a typical linear spline and terminates 


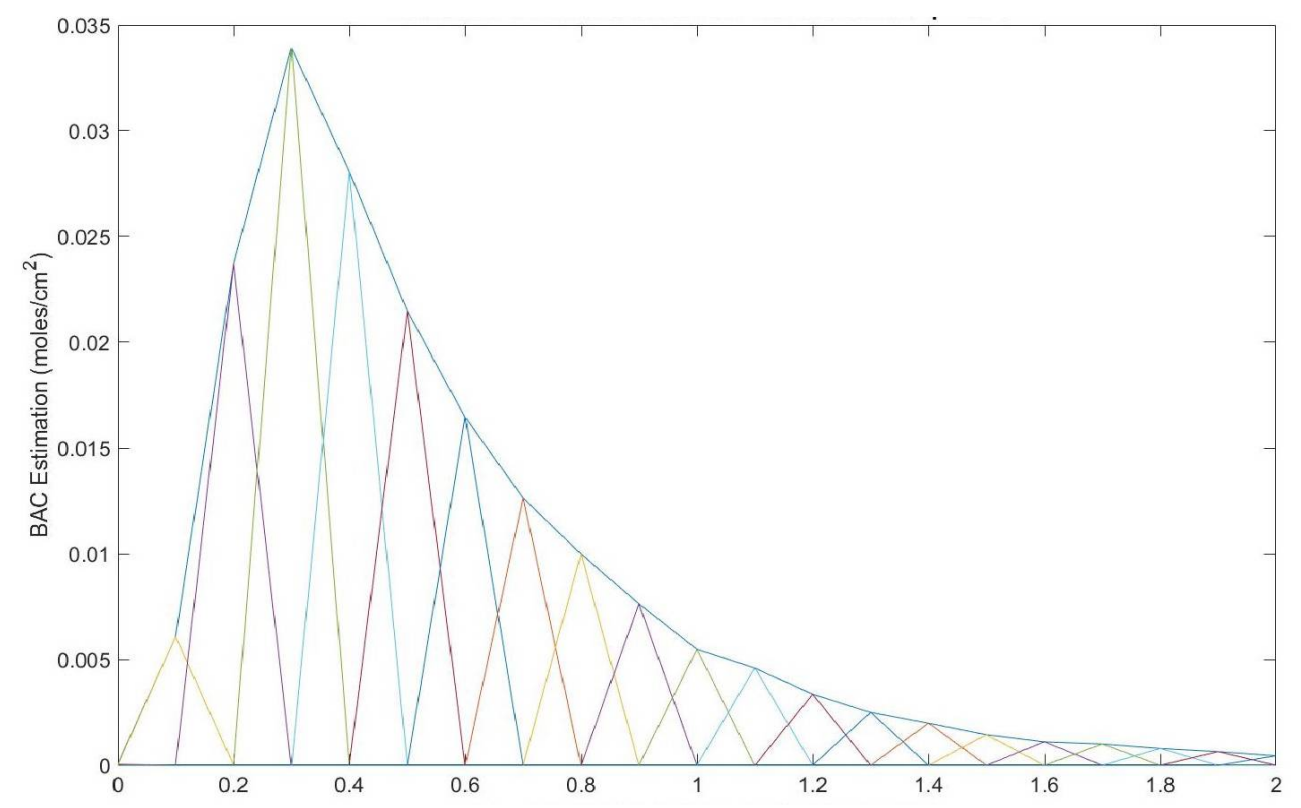

Figure 2.4: Example of B-Spline Curve-Fitting

at the knot located at $\eta=0$. We define it as

$$
\varphi_{0}(\eta)= \begin{cases}-N \eta+1 & \text { if } 0 \leq \eta<\frac{i}{N} \\ 0 & \text { elsewhere }\end{cases}
$$

The $N^{\text {th }}$ linear B-spline is unique from the others, due to how it only has the increasing line on the left and terminates at the knot located at $\eta=1$. We define it as

$$
\varphi_{N}(\eta)= \begin{cases}N \eta-N+1 & \text { if } \frac{N-1}{N} \leq \eta \leq 1 \\ 0 & \text { elsewhere }\end{cases}
$$


The graph of the linear combination of ordered linear B-spline bases when $N=5$ is shown in Figure 2.5. This figure clearly shows why the functions are sometimes described as a tent function due to the shape of they create on the support intervals.

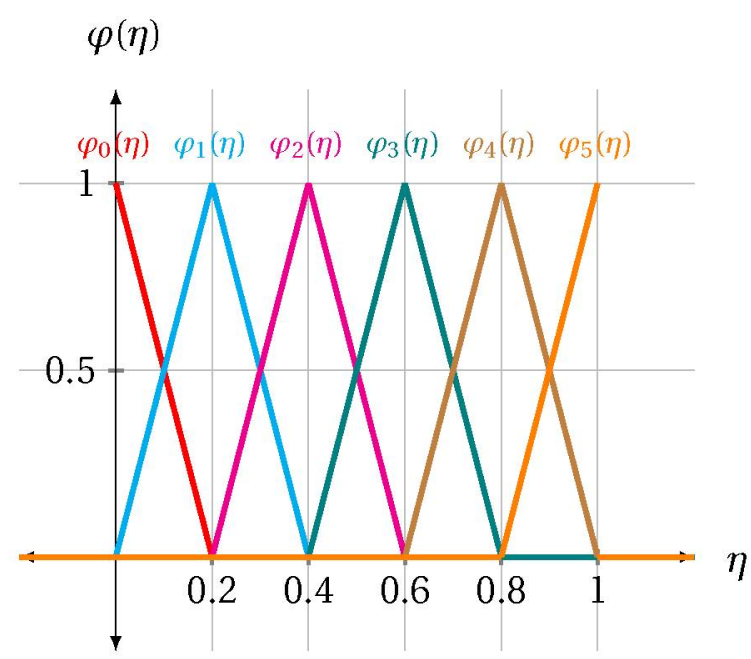

Figure 2.5: Example of linear B-Splines, $N=5$

Now that the test functions are well-defined as linear B-splines, they can be applied to Equation (2.11), giving us

$$
\begin{aligned}
\left\langle\frac{\partial u}{\partial t}(\eta, t), \varphi_{j}(\eta)\right\rangle_{V^{*}} & =\left\langle q_{1} \frac{\partial^{2} u}{\partial \eta^{2}}(\eta, t), \varphi_{j}(\eta)\right\rangle_{V^{*}} \\
& =\int_{0}^{1} q_{1} \frac{\partial^{2} u}{\partial \eta^{2}}(\eta, t) \varphi_{j}(\eta) \mathrm{d} \eta .
\end{aligned}
$$


Use integration by parts on the right-hand side, we obtain

$$
\begin{aligned}
\left\langle\frac{\partial u}{\partial t}(\eta, t), \varphi_{j}(\eta)\right\rangle_{V^{*}} & =\left.q_{1} \frac{\partial u}{\partial \eta}(\eta, t) \varphi_{j}(\eta)\right|_{0} ^{1}-\int_{0}^{1} q_{1} \frac{\partial u}{\partial \eta}(\eta, t) \frac{\partial \varphi_{j}}{\partial \eta}(\eta) \mathrm{d} \eta \\
& =q_{1} \frac{\partial u}{\partial \eta}(1, t) \varphi_{j}(1)-q_{1} \frac{\partial u}{\partial \eta}(0, t) \varphi_{j}(0) \\
& -\left\langle q_{1} \frac{\partial u}{\partial \eta}(\eta, t), \frac{\partial \varphi_{j}}{\partial \eta}(\eta)\right\rangle_{V^{*}}
\end{aligned}
$$

Next, applying the boundary conditions in Equations (2.9) and (2.10) to Equation (2.15), yields

$$
\begin{aligned}
\left\langle\frac{\partial u}{\partial t}(\eta, t), \varphi_{j}(\eta)\right\rangle_{V^{*}} & =q_{2} \alpha(t) \varphi_{j}(1)-u(0, t) \varphi_{j}(0) \\
& -q_{1}\left\langle\frac{\partial u}{\partial \eta}(\eta, t), \frac{\partial \varphi}{\partial \eta}(\eta)\right\rangle_{V^{*}}
\end{aligned}
$$

Let $\hat{u}(t ; q) \in V$ be a $(N+1) \times 1$ vector of $x$-valued points sampled at each respective $i^{t h}$ depth of $\eta$. The $i^{t h}$ entry of $\hat{u}(t ; q)$ is denoted as $u_{i}^{N}(t ; q)$ which represents the function value of $u(\eta, t ; q)$ at the $i^{t h}$ spline knot. Observe that $u(\eta, t ; q)$ can be approximated as an inner product between $\hat{u}(t ; q)$ and $\hat{\varphi}(\eta)$ by defining

$$
u^{N}(\eta, t ; q)=\langle\hat{u}(t ; q), \hat{\varphi}(\eta)\rangle_{V^{*}}=\sum_{i=0}^{N} u_{i}^{N}(t ; q) \varphi_{i}(\eta)
$$

Now use Equation (2.17) to calculate $\frac{\partial u^{N}}{\partial t}(\eta, t ; q)$ and $\frac{\partial u^{N}}{\partial \eta}(\eta, t ; q)$, which are the $\eta$ discretized approximations of $\frac{\partial u}{\partial t}(\eta, t ; q)$ and $\frac{\partial u}{\partial \eta}(\eta, t ; q)$. These calcula- 
tions below are required to evaluate the inner products above in the $\eta$ discretized heat equation shown in Equation (2.16).

$$
\begin{aligned}
\frac{\partial u^{N}}{\partial t}(\eta, t ; q) & =\frac{\partial}{\partial t}\left(\sum_{i=0}^{N} u_{i}^{N}(t ; q) \varphi_{i}(\eta)\right) \\
& =\sum_{i=0}^{N} \frac{\partial}{\partial t}\left(u_{i}^{N}(t ; q) \varphi_{i}(\eta)\right) \\
& =\sum_{i=0}^{N} \frac{\partial u_{i}^{N}}{\partial t}(t ; q) \varphi_{i}(\eta)
\end{aligned}
$$

and

$$
\begin{aligned}
\frac{\partial u^{N}}{\partial \eta}(\eta, t ; q) & =\frac{\partial}{\partial \eta}\left(\sum_{i=0}^{N} u_{i}^{N}(t ; q) \varphi_{i}(\eta)\right) \\
& =\sum_{i=0}^{N} \frac{\partial}{\partial \eta}\left(u_{i}^{N}(t ; q) \varphi_{i}(\eta)\right) \\
& =\sum_{i=0}^{N} u_{i}^{N}(t ; q) \frac{\partial \varphi_{i}}{\partial \eta}(\eta) .
\end{aligned}
$$

Apply the Equation (2.17) spline approximation of $u(\eta, t ; q)$ and the Equation (2.18) and Equation (2.19) spline approximations of $\frac{\partial u}{\partial t}(\eta, t)$ and $\frac{\partial u}{\partial \eta}(\eta, t)$ respectively to Equation (2.16).

$$
\begin{aligned}
\left\langle\sum_{i=0}^{N} \frac{\partial u_{i}^{N}}{\partial t}(t ; q) \varphi_{i}(\eta), \varphi_{j}(\eta)\right\rangle_{V^{*}} & =q_{2} \alpha(t) \varphi_{j}(1)-\sum_{i=0}^{N} u_{i}^{N}(t ; q) \varphi_{i}(0) \varphi_{j}(0) \\
& -q_{1}\left\langle\sum_{i=0}^{N} u_{i}^{N}(t ; q) \frac{\partial \varphi_{i}}{\partial \eta}(\eta), \frac{\partial \varphi_{j}}{\partial \eta}(\eta)\right\rangle_{V^{*}}
\end{aligned}
$$


The linearity property of inner products can be applied to Equation (2.20).

$$
\begin{aligned}
\sum_{i=0}^{N} \frac{\partial u_{i}^{N}}{\partial t}(t ; q) \cdot\left\langle\varphi_{i}(\eta), \varphi_{j}(\eta)\right\rangle_{V^{*}} & =q_{2} \alpha(t) \varphi_{j}(1)-\sum_{i=0}^{N} u_{i}^{N}(t ; q) \varphi_{i}(0) \varphi_{j}(0) \\
& -q_{1} \sum_{i=0}^{N} u_{i}^{N}(t ; q) \cdot\left\langle\frac{\partial \varphi_{i}}{\partial \eta}(\eta), \frac{\partial \varphi_{j}}{\partial \eta}(\eta)\right\rangle_{V^{*}}
\end{aligned}
$$

Now, we evaluate the inner products between spline functions in Equation (2.21). The inner product is defined on $V^{*}$, since these functions belong to the space of test functions (see Definition A.2.9), is calculated as

$$
\langle\varphi, \psi\rangle_{V^{*}}=\int_{a}^{b} \varphi(\eta) \psi(\eta) \mathrm{d} \eta
$$

The integrals have a few possible results, depending on how the B-spline functions overlap in intervals of support. The integral equals zero elsewhere if even one of the two functions is zero on a particular interval.

First, consider the inner product of a spline with itself. For $i=1, \ldots, N-1$ we have

$$
\left\langle\varphi_{i}(\eta), \varphi_{i}(\eta)\right\rangle_{V^{*}}=\int_{\frac{i-1}{N}}^{\frac{i+1}{N}}\left(\varphi_{i}(\eta)\right)^{2} \mathrm{~d} \eta
$$

The graph of this case is in Figure 2.6. 


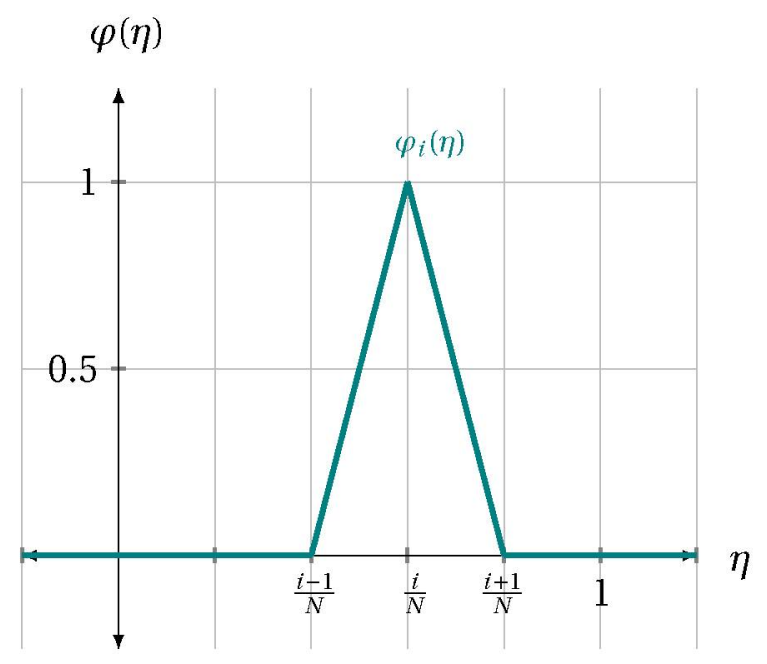

Figure 2.6: Plot of $\varphi_{i}(\eta)$

Since the inner product result is only nonzero on $\left[\frac{i-1}{N}, \frac{i+1}{N}\right]$, we have

$$
\begin{aligned}
\left\langle\varphi_{i}(\eta), \varphi_{i}(\eta)\right\rangle_{V^{*}} & =\int_{\frac{i-1}{N}}^{\frac{i}{N}}\left(\varphi_{i}(\eta)\right)^{2} \mathrm{~d} \eta+\int_{\frac{i}{N}}^{\frac{i+1}{N}}\left(\varphi_{i}(\eta)\right)^{2} \mathrm{~d} \eta \\
& =\int_{\frac{i-1}{N}}^{\frac{i}{N}}(N \eta-i+1)^{2} \mathrm{~d} \eta+\int_{\frac{i}{N}}^{\frac{i+1}{N}}(-N \eta+i+1)^{2} \mathrm{~d} \eta \\
& =\frac{4}{6 N}
\end{aligned}
$$

The next case considers the inner product of a spline basis element with a directly adjacent spline basis element to its right, defined for the values $i=$ $0, \ldots, N-1$,

$$
\left\langle\varphi_{i}(\eta), \varphi_{i+1}(\eta)\right\rangle_{V^{*}}=\int_{\frac{i-1}{N}}^{\frac{i+2}{N}} \varphi_{i}(\eta) \cdot \varphi_{i+1}(\eta) \mathrm{d} \eta
$$

Observe the graphical representation of this case in Figure 2.7. 


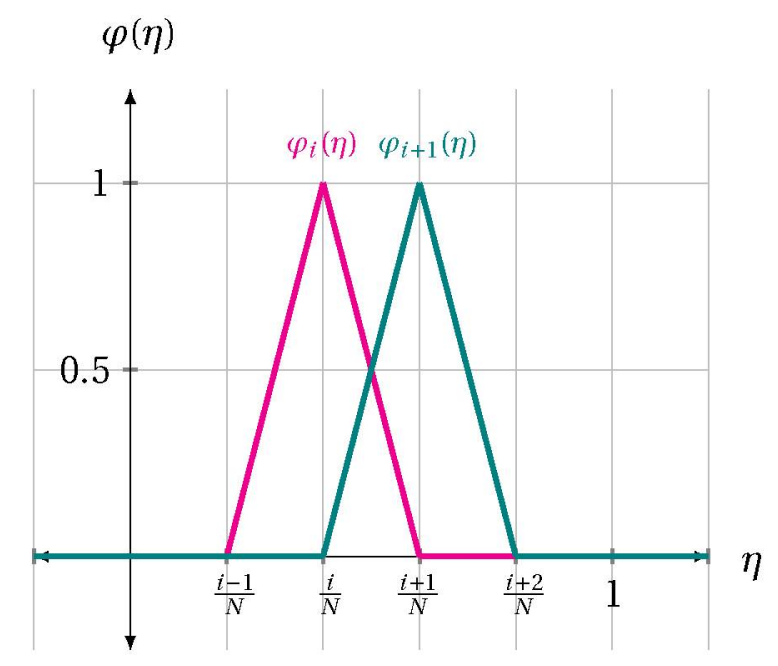

Figure 2.7: Plot of $\varphi_{i}(\eta)$ and $\varphi_{i+1}(\eta)$

Since the inner product result is only nonzero on $\left[\frac{i}{N}, \frac{i+1}{N}\right]$, it reduces to

$$
\begin{aligned}
\left\langle\varphi_{i}(\eta), \varphi_{i+1}(\eta)\right\rangle_{V^{*}} & =\int_{\frac{i}{N}}^{\frac{i+1}{N}} \varphi_{i}(\eta) \cdot \varphi_{i+1}(\eta) \mathrm{d} \eta \\
& =\int_{\frac{i}{N}}^{\frac{i+1}{N}}(-N \eta+i+1)(N \eta-(i+1)+1) \mathrm{d} \eta \\
& =\frac{1}{6 N} .
\end{aligned}
$$

This calculation also considers the case $\left\langle\varphi_{i}(\eta), \varphi_{i-1}(\eta)\right\rangle_{V}$. Choose $i=k+1$ and this gives $\left\langle\varphi_{i-1}(\eta), \varphi_{i}(\eta)\right\rangle_{V}=\left\langle\varphi_{k}(\eta), \varphi_{k+1}(\eta)\right\rangle_{V}$

An important observation is that the inner product is zero for $\left\langle\varphi_{i}(\eta), \varphi_{j}(\eta)\right\rangle_{V}$ when $j \neq i$ or $j \neq i \pm 1$, because the spline bases do not overlap in a support interval. Therefore,

$$
\left\langle\varphi_{i}(\eta), \varphi_{j}(\eta)\right\rangle_{V^{*}}=0 \quad \text { when } j \neq i \text { or } j \neq i \pm 1 \text {. }
$$


There is a special case when $i=0$ for both spline bases. Here only the decreasing portion on the right half of the the knot located at $\eta=0$ is in the support interval. Then the inner product reduces to

$$
\left\langle\varphi_{0}(\eta), \varphi_{0}(\eta)\right\rangle_{V^{*}}=\int_{0}^{\frac{1}{N}}\left(\varphi_{0}(\eta)\right)^{2} \mathrm{~d} \eta
$$

See Figure 2.8 for a graphical representation of the B-spline.

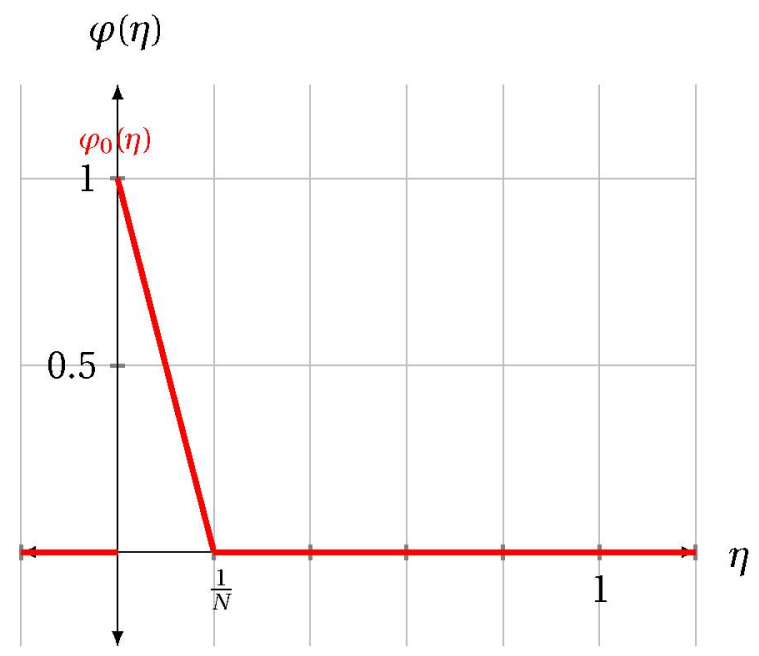

Figure 2.8: Plot of $\varphi_{0}(\eta)$

Since the inner product result is only nonzero on $\left[0, \frac{1}{N}\right]$, we obtain

$$
\begin{aligned}
\left\langle\varphi_{0}(\eta), \varphi_{0}(\eta)\right\rangle_{V^{*}} & =\int_{0}^{\frac{1}{N}}\left(\varphi_{0}(\eta)\right)^{2} \mathrm{~d} \eta \\
& =\int_{0}^{\frac{1}{N}}(-N \eta+1)^{2} \mathrm{~d} \eta \\
& =\frac{2}{6 N} .
\end{aligned}
$$


The last case considers when $i=N$ for both spline bases. This is a special case where only the increasing portion on the left half of the knot located at $\eta=1$ is in the support interval. Then the inner product is given by

$$
\left\langle\varphi_{N}(\eta), \varphi_{N}(\eta)\right\rangle_{V^{*}}=\int_{\frac{N-1}{N}}^{1}\left(\varphi_{N}(\eta)\right)^{2} \mathrm{~d} \eta
$$

See Figure 2.9 for a graphical representation of the B-spline.

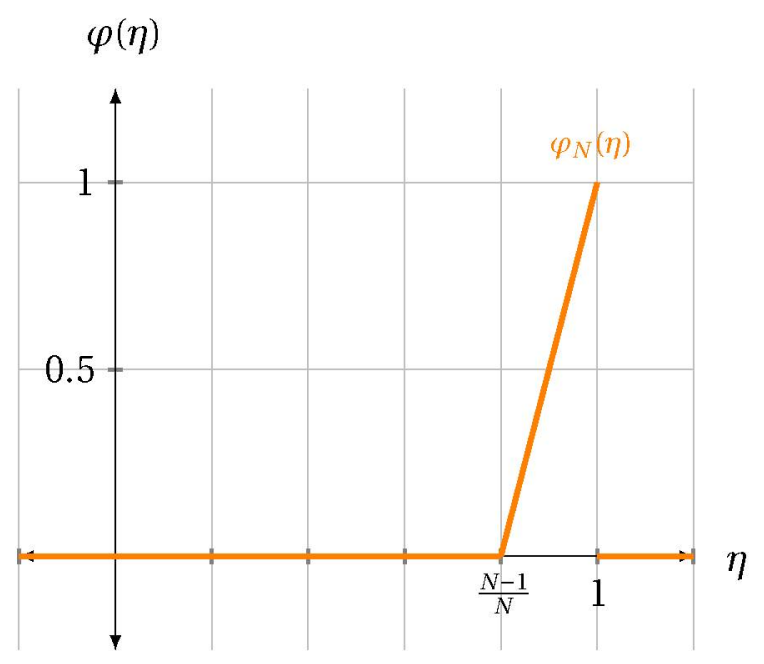

Figure 2.9: Plot of $\varphi_{N}(\eta)$

Since, the inner product result is only nonzero on $\left[\frac{N-1}{N}, 1\right]$, we obtain

$$
\begin{aligned}
\left\langle\varphi_{N}(\eta), \varphi_{N}(\eta)\right\rangle_{V^{*}} & =\int_{\frac{N-1}{N}}^{1}\left(\varphi_{N}(\eta)\right)^{2} \mathrm{~d} \eta \\
& \left.=\int_{\frac{N-1}{N}}^{1}(N \eta-N+1)\right)^{2} \mathrm{~d} \eta \\
& =\frac{2}{6 N}
\end{aligned}
$$


We now summarize the results of the calculations in Equations (2.24), (2.26), (2.27), (2.29), and (2.31), which display all possible inner product combinations of two linear B-splines:

$$
\begin{aligned}
\left\langle\varphi_{i}(\eta), \varphi_{i}(\eta)\right\rangle_{V^{*}} & =\frac{4}{6 N} \\
\left\langle\varphi_{i}(\eta), \varphi_{i \pm 1}(\eta)\right\rangle_{V^{*}} & =\frac{1}{6 N} \\
\left\langle\varphi_{0}(\eta), \varphi_{0}(\eta)\right\rangle_{V^{*}} & =\frac{2}{6 N} \\
\left\langle\varphi_{N}(\eta), \varphi_{N}(\eta)\right\rangle_{V^{*}} & =\frac{2}{6 N} \\
\left\langle\varphi_{i}(\eta), \varphi_{j}(\eta)\right\rangle_{V^{*}} & =0 \quad \text { when } j \neq i \text { or } j \neq i \pm 1
\end{aligned}
$$

Collecting these results into an $(N+1) \times(N+1)$ matrix gives

$$
M=\frac{1}{6 N}\left[\begin{array}{cccccccc}
2 & 1 & 0 & 0 & \ldots & \ldots & 0 & 0 \\
1 & 4 & 1 & 0 & \ldots & \ldots & 0 & 0 \\
0 & 1 & 4 & \ddots & \ddots & & \vdots & \vdots \\
0 & 0 & \ddots & \ddots & & \ddots & \vdots & \vdots \\
\vdots & \vdots & \ddots & & \ddots & \ddots & 0 & 0 \\
\vdots & \vdots & & \ddots & \ddots & 4 & 1 & 0 \\
0 & 0 & \ldots & \ldots & 0 & 1 & 4 & 1 \\
0 & 0 & \ldots & \ldots & 0 & 0 & 1 & 2
\end{array}\right]
$$


This $M$ matrix is utilized later to express the discretized heat equation solution as a matrix.

Recall that the goal of these calculations is to find the inner products in Equation (2.21) shown below:

$$
\begin{aligned}
\sum_{i=0}^{N} \frac{\partial u_{i}^{N}}{\partial t}(t ; q)\left\langle\varphi_{i}(\eta), \varphi_{j}(\eta)\right\rangle_{V^{*}} & =q_{2} \alpha(t) \varphi_{j}(1)-\sum_{i=0}^{N} u_{i}^{N}(t ; q) \varphi_{i}(0) \varphi_{j}(0) \\
& -q_{1} \sum_{i=0}^{N} u_{i}^{N}(t ; q)\left\langle\frac{\partial \varphi_{i}}{\partial \eta}(\eta), \frac{\partial \varphi_{j}}{\partial \eta}(\eta)\right\rangle_{V^{*}}
\end{aligned}
$$

We already calculated the inner product cases involving the linear B-splines on the left-hand side. We now perform the calculations involving the inner products of the partial derivatives of the linear B-spline functions on the righthand side. Define the $i^{t h}$ linear B-spline derivative function, for $i=1, \ldots, N-1$ as

$$
\frac{\partial \varphi_{i}}{\partial \eta}(\eta)= \begin{cases}N & \text { if } \frac{i-1}{N} \leq \eta<\frac{i}{N} \\ -N & \text { if } \frac{i}{N} \leq \eta<\frac{i+1}{N} \\ 0 & \text { elsewhere }\end{cases}
$$

The $0^{\text {th }}$ linear B-spline derivative function is unique from the others due to how it only has the negative portion on the right of the knot located at $\eta=0$. 
It is defined below

$$
\frac{\partial \varphi_{0}}{\partial \eta}(\eta)= \begin{cases}-N & \text { if } 0 \leq \eta<\frac{1}{N} \\ 0 & \text { elsewhere }\end{cases}
$$

The $N^{t h}$ linear B-spline derivative function is unique from the others due to how it only has the positive $N$ portion on the left of the knot located at $\eta=1$. It is defined below

$$
\frac{\partial \varphi_{N}}{\partial \eta}(\eta)= \begin{cases}N & \text { if } \frac{N-1}{N} \leq \eta \leq 1 \\ 0 & \text { elsewhere }\end{cases}
$$

The inner product calculations are similar to the previous ones, in that they have the same cases with different functions. See Figure 2.10 for an example of the spline derivative bases for $N=5$.

First, consider the inner product of a spline basis function derivative with itself. For $i=1, \ldots, N-1$ we have

$$
\left\langle\frac{\partial \varphi_{i}}{\partial \eta}(\eta), \frac{\partial \varphi_{i}}{\partial \eta}(\eta)\right\rangle_{V^{*}}=\int_{\frac{i-1}{N}}^{\frac{i+1}{N}}\left(\frac{\partial \varphi_{i}}{\partial \eta}(\eta)\right)^{2} \mathrm{~d} \eta
$$

The plot is shown in Figure 2.11. 


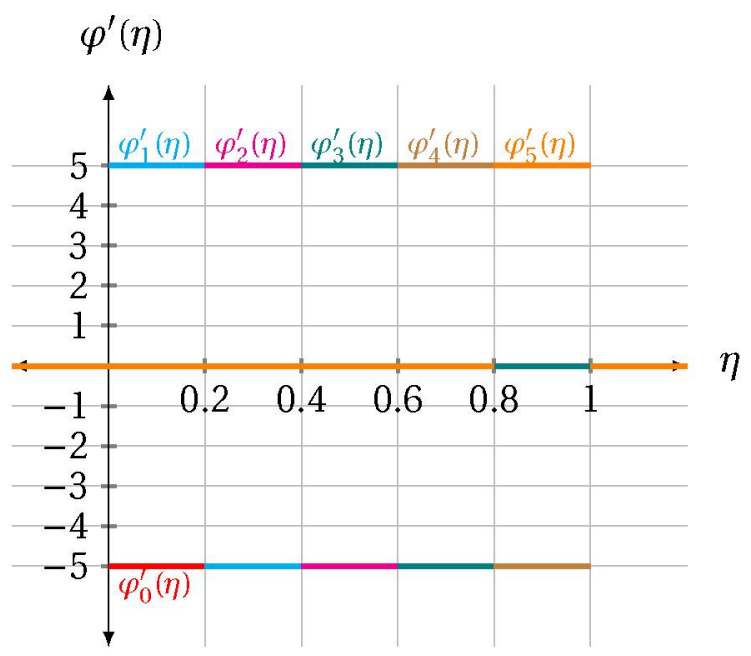

Figure 2.10: Example of partials of linear B-Splines, $N=5$

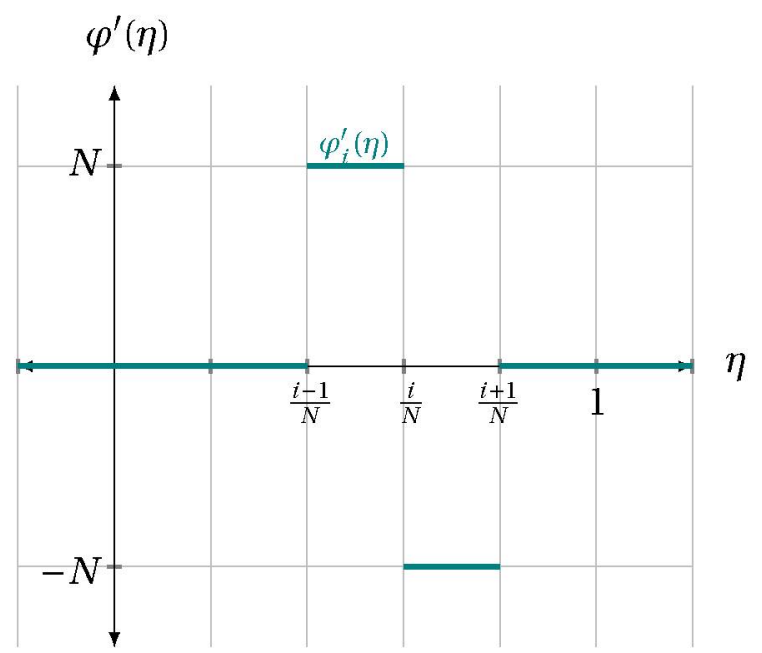

Figure 2.1 1: Plot of $\frac{\partial \varphi_{i}}{\partial \eta}(\eta)$ 
Since the inner product result is only nonzero on $\left[\frac{i-1}{N}, \frac{i+1}{N}\right]$, we obtain

$$
\begin{aligned}
\left\langle\frac{\partial \varphi_{i}}{\partial \eta}(\eta), \frac{\partial \varphi_{i}}{\partial \eta}(\eta)\right\rangle_{V^{*}} & =\int_{\frac{i-1}{N}}^{\frac{i}{N}}\left(\frac{\partial \varphi_{i}}{\partial \eta}(\eta)\right)^{2} \mathrm{~d} \eta+\int_{\frac{i}{N}}^{\frac{i+1}{N}}\left(\frac{\partial \varphi_{i}}{\partial \eta}(\eta)\right)^{2} \mathrm{~d} \eta \\
& =\int_{\frac{i-1}{N}}^{\frac{i}{N}}(N)^{2} \mathrm{~d} \eta+\int_{\frac{i}{N}}^{\frac{i+1}{N}}(-N)^{2} \mathrm{~d} \eta \\
& =\int_{\frac{i-1}{N}}^{\frac{i+1}{N}} N^{2} \mathrm{~d} \eta \\
& =2 N .
\end{aligned}
$$

Second, consider the inner product of a spline basis derivative with a directly adjacent spline basis derivative to its right.

$$
\left\langle\frac{\partial \varphi_{i}}{\partial \eta}(\eta), \frac{\partial \varphi_{i+1}}{\partial \eta}(\eta)\right\rangle_{V^{*}}=\int_{\frac{i-1}{N}}^{\frac{i+2}{N}} \frac{\partial \varphi_{i}}{\partial \eta}(\eta) \cdot \frac{\partial \varphi_{i+1}}{\partial \eta}(\eta) \mathrm{d} \eta
$$

The plot is shown in Figure 2.12.

Since the inner product result is only nonzero on $\left[\frac{i}{N}, \frac{i+1}{N}\right]$, we obtain

$$
\begin{aligned}
\left\langle\frac{\partial \varphi_{i}}{\partial \eta}(\eta), \frac{\partial \varphi_{i+1}}{\partial \eta}(\eta)\right\rangle_{V^{*}} & =\int_{\frac{i}{N}}^{\frac{i+1}{N}} \frac{\partial \varphi_{i}}{\partial \eta}(\eta) \cdot \frac{\partial \varphi_{i+1}}{\partial \eta}(\eta) \mathrm{d} \eta \\
& =\int_{\frac{i}{N}}^{\frac{i+1}{N}}(-N) \cdot(N) \mathrm{d} \eta \\
& =\int_{\frac{i}{N}}^{\frac{i+1}{N}}-N^{2} \mathrm{~d} \eta \\
& =-N .
\end{aligned}
$$

As in the linear B-spline inner products, this calculation also considers the case $\left\langle\frac{\partial \varphi_{i-1}}{\partial \eta}(\eta), \frac{\partial \varphi_{i}}{\partial \eta}(\eta)\right\rangle_{V^{*}}$. Choose $i=k+1$ and this gives $\left\langle\frac{\partial \varphi_{i-1}}{\partial \eta}(\eta), \frac{\partial \varphi_{i}}{\partial \eta}(\eta)\right\rangle_{V^{*}}=$ 


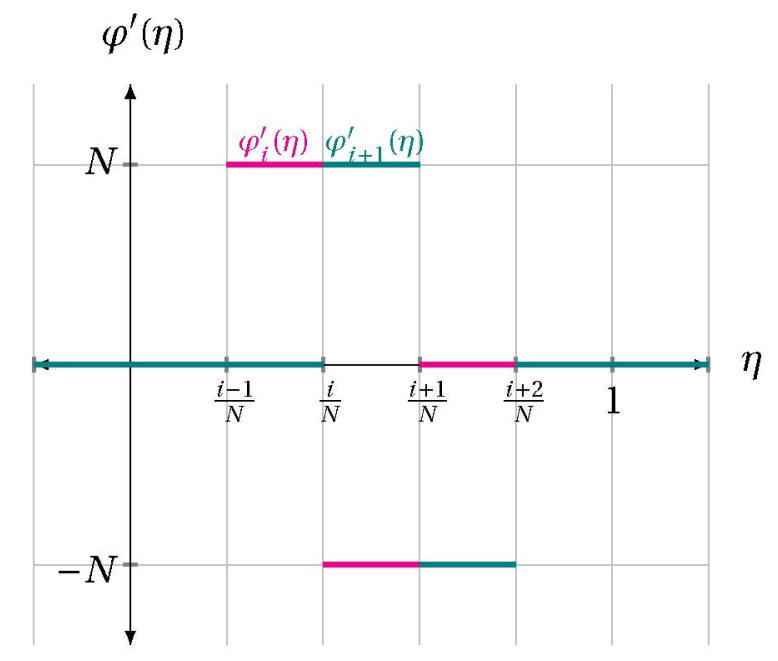

Figure 2.12: $\operatorname{Plot}$ of $\frac{\partial \varphi_{i}}{\partial \eta}(\eta)$ and $\frac{\partial \varphi_{i+1}}{\partial \eta}(\eta)$

$\left\langle\frac{\partial \varphi_{k}}{\partial \eta}(\eta), \frac{\partial \varphi_{k+1}}{\partial \eta}(\eta)\right\rangle_{V^{*}}$

The inner product is zero for $\left\langle\frac{\partial \varphi_{i}}{\partial \eta}(\eta), \frac{\partial \varphi_{j}}{\partial \eta}(\eta)\right\rangle_{V^{*}}$ when $j \neq i$ or $j \neq i \pm 1$ since the splines will not overlap in support interval. Therefore,

$$
\left\langle\frac{\partial \varphi_{i}}{\partial \eta}(\eta), \frac{\partial \varphi_{j}}{\partial \eta}(\eta)\right\rangle_{V^{*}}=0 \quad \text { when } j \neq i \text { or } j \neq i \pm 1
$$

There is a special case when $i=0$ for both spline derivative bases. Here only the negative portion on the right half of the the discontinuity located at $\eta=0$ is in the support interval. Then the inner product reduces to

$$
\left\langle\frac{\partial \varphi_{0}}{\partial \eta}(\eta), \frac{\partial \varphi_{0}}{\partial \eta}(\eta)\right\rangle_{V^{*}}=\int_{0}^{\frac{1}{N}}\left(\frac{\partial \varphi_{0}}{\partial \eta}(\eta)\right)^{2} \mathrm{~d} \eta
$$




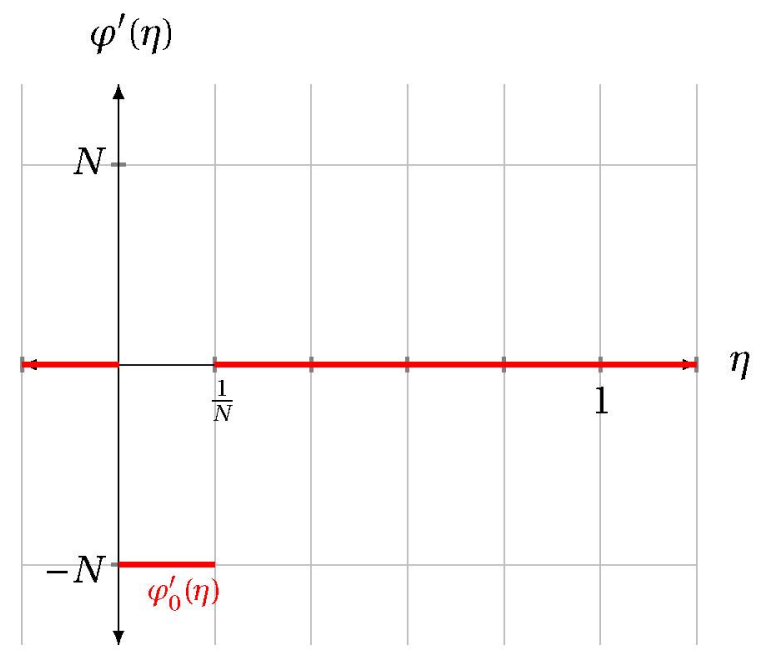

Figure 2.13: Plot of $\frac{\partial \varphi_{0}}{\partial \eta}(\eta)$

The plot is shown in Figure 2.13. Since the inner product result is only nonzero on $\left[0, \frac{1}{N}\right]$, we obtain

$$
\begin{aligned}
\left\langle\frac{\partial \varphi_{0}}{\partial \eta}(\eta), \frac{\partial \varphi_{0}}{\partial \eta}(\eta)\right\rangle_{V^{*}} & =\int_{0}^{\frac{1}{N}}\left(\frac{\partial \varphi_{0}}{\partial \eta}(\eta)\right)^{2} \mathrm{~d} \eta \\
& =\int_{0}^{\frac{1}{N}}(-N)^{2} \mathrm{~d} \eta \\
& =N .
\end{aligned}
$$

Finally, we consider a special case when $i=N$ for both spline derivative bases. Here only the positive portion on the left half of the the discontinuity located at $\eta=1$ is in the support interval. Then the inner product reduces to

$$
\left\langle\frac{\partial \varphi_{N}}{\partial \eta}(\eta), \frac{\partial \varphi_{N}}{\partial \eta}(\eta)\right\rangle_{V^{*}}=\int_{\frac{N-1}{N}}^{1}\left(\frac{\partial \varphi_{N}}{\partial \eta}(\eta)\right)^{2} \mathrm{~d} \eta
$$




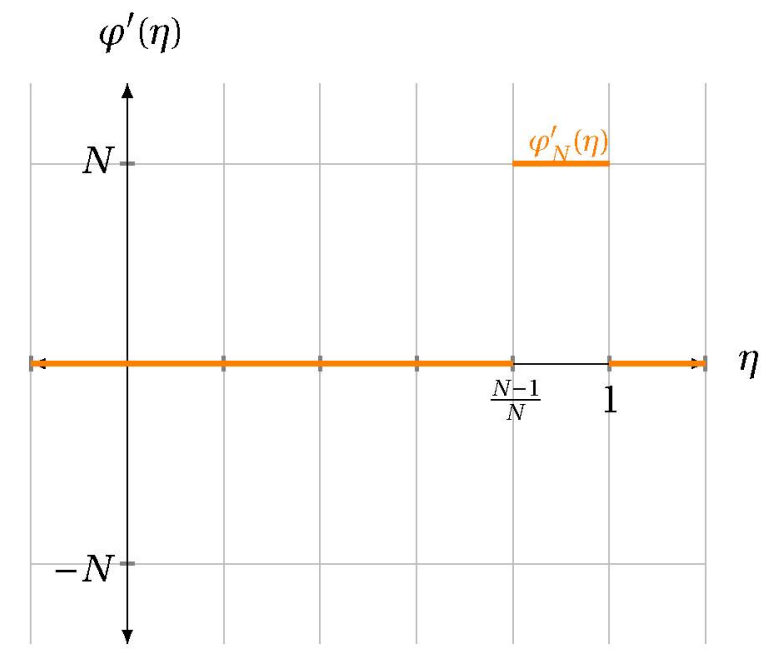

Figure 2.14: Plot of $\frac{\partial \varphi_{N}}{\partial \eta}(\eta)$

The plot is shown in Figure 2.14.

Since the inner product result is only nonzero on $\left[\frac{N-1}{N}, 1\right]$, we obtain

$$
\begin{aligned}
\left\langle\frac{\partial \varphi_{N}}{\partial \eta}(\eta), \frac{\partial \varphi_{N}}{\partial \eta}(\eta)\right\rangle_{V^{*}} & =\int_{\frac{N-1}{N}}^{1}\left(\frac{\partial \varphi_{N}}{\partial \eta}(\eta)\right)^{2} \mathrm{~d} \eta \\
& =\int_{\frac{N-1}{N}}^{1} N^{2} \mathrm{~d} \eta \\
& =N .
\end{aligned}
$$

In summary, the results of the calculations in Equations (2.42), (2.44), (2.45), (2.47), and (2.49) display the all possible inner product combinations of the 
partial derivatives of the linear B-splines are shown below:

$$
\begin{aligned}
& \left\langle\frac{\partial \varphi_{i}}{\partial \eta}(\eta), \frac{\partial \varphi_{i}}{\partial \eta}(\eta)\right\rangle_{V^{*}}=2 N \\
& \left\langle\frac{\partial \varphi_{i}}{\partial \eta}(\eta), \frac{\partial \varphi_{i \pm 1}}{\partial \eta}(\eta)\right\rangle_{V^{*}}=-N \\
& \left\langle\frac{\partial \varphi_{0}}{\partial \eta}(\eta), \frac{\partial \varphi_{0}}{\partial \eta}(\eta)\right\rangle_{V^{*}}=N \\
& \left\langle\frac{\partial \varphi_{N}}{\partial \eta}(\eta), \frac{\partial \varphi_{N}}{\partial \eta}(\eta)\right\rangle_{V^{*}}=N \\
& \left\langle\frac{\partial \varphi_{i}}{\partial \eta}(\eta), \frac{\partial \varphi_{j}}{\partial \eta}(\eta)\right\rangle_{V^{*}}=0 \quad \text { when } j \neq i \text { or } j \neq i \pm 1
\end{aligned}
$$

Collecting these results into an $(N+1) \times(N+1)$ matrix gives

$$
K=N\left[\begin{array}{cccccccc}
1 & -1 & 0 & 0 & \ldots & \ldots & 0 & 0 \\
-1 & 2 & -1 & 0 & \ldots & \ldots & 0 & 0 \\
0 & -1 & 2 & \ddots & \ddots & & \vdots & \vdots \\
0 & 0 & \ddots & \ddots & & \ddots & \vdots & \vdots \\
\vdots & \vdots & \ddots & & \ddots & \ddots & 0 & 0 \\
\vdots & \vdots & & \ddots & \ddots & 2 & -1 & 0 \\
0 & 0 & \ldots & \ldots & 0 & -1 & 2 & -1 \\
0 & 0 & \ldots & \ldots & 0 & 0 & -1 & 1
\end{array}\right]
$$

This $K$ matrix is utilized later to express the discretized heat equation solution as a matrix. 
Now that we have completed the inner product calculations between two linear B-spline functions and the inner product calculations between two linear B-spline partial derivatives, we can now revisit the terms of Equation (2.21) shown below.

$$
\begin{aligned}
\sum_{i=0}^{N} \frac{\partial u_{i}^{N}}{\partial t}(t ; q) \cdot\left\langle\varphi_{i}(\eta), \varphi_{j}(\eta)\right\rangle_{V^{*}} & =q_{2} \alpha(t) \varphi_{j}(1)-\sum_{i=0}^{N} u_{i}^{N}(t ; q) \varphi_{i}(0) \varphi_{j}(0) \\
& -q_{1} \sum_{i=0}^{N} u_{i}^{N}(t ; q) \cdot\left\langle\frac{\partial \varphi_{i}}{\partial \eta}(\eta), \frac{\partial \varphi_{j}}{\partial \eta}(\eta)\right\rangle_{V^{*}}
\end{aligned}
$$

The matrices $M$ in Equation (2.37) and $K$ in Equation (2.55) take into account the entire B-spline and B-spline partial derivative vectors. If Equation (2.21) is to be calculated, all terms must also be similarly converted into their respective vector forms at this time. Note that variables with a "hat" denote vectors.

Begin by considering the first term of the right-hand side of Equation (2.21). Refer back to Equations (2.12), (2.13), and (2.14) for the B-spline functions to note that $\varphi_{j}(1)=0$ for all $j \neq N$ and $\varphi_{N}(1)=1$. The coordinates together give 
the $(N+1) \times 1$ vector

$$
\hat{\varphi}(1)=\left[\begin{array}{l}
0 \\
0 \\
\vdots \\
0 \\
1
\end{array}\right] .
$$

Now define

$$
B=\hat{\varphi}(1)
$$

Then it follows that

$$
\left\{q_{2} \alpha(t) \varphi_{j}(1)\right\}_{j=0}^{N}=q_{2} \alpha(t) \hat{\varphi}(1)=q_{2} \alpha(t) B
$$

Next we rewrite the second term of the right-hand side of Equation (2.21) as follows. Define $\hat{u}^{N}(t ; q)$ to be the $(N+1) \times 1$ vector with entries $u_{i}^{N}(t ; q)$ for $i=0,1, \ldots, N$. Refer back to Equations (2.12), (2.13), and (2.14) for the B-spline functions to note that $\varphi_{j}(0)=0$ for all $j \neq 0$ and $\varphi_{0}(0)=1$. The combination of the spline products and the alcohol concentration measurement vector re- 
sults in Equation (2.59).

$$
\left\{\sum_{i=0}^{N} u_{i}^{N}(t ; q) \varphi_{i}(0) \varphi_{j}(0)\right\}_{i, j=0}^{N}=\left[\begin{array}{cccccccc}
1 & 0 & 0 & 0 & \ldots & \ldots & 0 & 0 \\
0 & 0 & 0 & 0 & \ldots & \ldots & 0 & 0 \\
0 & 0 & 0 & \ddots & \ddots & & \vdots & \vdots \\
0 & 0 & \ddots & \ddots & & \ddots & \vdots & \vdots \\
\vdots & \vdots & \ddots & & \ddots & \ddots & 0 & 0 \\
\vdots & \vdots & & \ddots & \ddots & 0 & 0 & 0 \\
0 & 0 & \ldots & \ldots & 0 & 0 & 0 & 0 \\
0 & 0 & \ldots & \ldots & 0 & 0 & 0 & 0
\end{array}\right]\left[\begin{array}{c}
u_{0}^{N}(t ; q) \\
u_{1}^{N}(t ; q) \\
\vdots \\
u_{N-1}^{N}(t ; q) \\
u_{N}^{N}(t ; q)
\end{array}\right]
$$

Now define the $(N+1) \times(N+1)$ matrix

$$
R=\left\{\varphi_{i}(0) \varphi_{j}(0)\right\}_{i, j=0}^{N}
$$

Then it follows that

$$
\left\{\sum_{i=0}^{N} u_{i}^{N}(t ; q) \varphi_{i}(0) \varphi_{j}(0)\right\}_{i, j=0}^{N} \equiv R \hat{u}^{N}(t ; q) .
$$

Now define $\frac{\partial \hat{u}^{N}}{\partial t}(t ; q)$ to be the $(N+1) \times 1$ vector of discretized partials of $u(\eta, t ; q)$ with respect to $\eta$ with entries $\frac{\partial u_{i}^{N}}{\partial t}(t ; q)$ for $i=0,1, \ldots, N$. Take the previous results of $M$ from Equation (2.37), $K$ from Equation (2.55), the vector $\frac{\partial \hat{u}^{N}}{\partial t}(t ; q)$, the vector $\hat{u}^{N}(t ; q), B$ from Equation (2.57), and $R \hat{u}^{N}(t ; q)$ from 
Equation (2.61) and apply them to Equation (2.21) giving us

$$
\begin{aligned}
M \frac{\partial \hat{u}^{N}}{\partial t}(t ; q) & =q_{2} \alpha(t) B-R \hat{u}^{N}(t ; q)-q_{1} K \hat{u}^{N}(t ; q) \\
M \frac{\partial \hat{u}^{N}}{\partial t}(t ; q) & =q_{2} \alpha(t) B+\left(-R-q_{1} K\right) \hat{u}^{N}(t ; q) \\
\frac{\partial \hat{u}^{N}}{\partial t}(t ; q) & =\alpha(t) q_{2} M^{-1} B+M^{-1}\left(-R-q_{1} K\right) \hat{u}^{N}(t ; q) .
\end{aligned}
$$

This completes the discretization of the heat equation in the weak formulation over the variable $\eta$ into $N$ uniform partitions. Note that $M$ is always invertible for $1 \leq N<\infty$, because $M$ is a symmetric and positive-definite matrix. It is important to observe that this new form of the equation no longer is dependent on $\eta$. The left-hand side of Equation (2.62) is now a vector dependent on time $t$ with parameters $q=\left\langle q_{1}, q_{2}\right\rangle$ which has values for $u(\eta, t ; q)$ at uniformly partitioned levels of $\eta$ where the vector index provides the depth. Defining $A(q)=M^{-1}\left(-R-q_{1} K\right)$ and $B(q)=q_{2} M^{-1} B$, this simplifies Equation (2.62) to

$$
\begin{aligned}
\frac{\partial \hat{u}^{N}}{\partial t}(t ; q) & =\alpha(t) B(q)+A(q) \hat{u}^{N}(t ; q) \\
\frac{\partial \hat{u}^{N}}{\partial t}(t ; q)-A(q) \hat{u}^{N}(t ; q) & =\alpha(t) B(q)
\end{aligned}
$$

Now we use the method of integrating factors to solve for $\hat{u}^{N}(t ; q)$. Assume $t \geq 0$. Then, multiplying both sides by the integrating factor

$$
\mu(t ; q)=e^{\int-A(q) \mathrm{d} t}=e^{-A(q) t}
$$


yields

$$
\begin{aligned}
\mu(t ; q) \cdot \frac{\partial \hat{u}^{N}}{\partial t}(t ; q)-\mu(t ; q) \cdot A(q) \hat{u}^{N}(t ; q) & =\mu(t ; q) \cdot \alpha(t) B(q) \\
e^{-A(q) t} \cdot \frac{\partial \hat{u}^{N}}{\partial t}(t ; q)-e^{-A(q) t} \cdot A(q) \hat{u}^{N}(t ; q) & =e^{-A(q) t} \cdot \alpha(t) B(q) \\
\mathrm{d}\left(e^{-A(q) t} \hat{u}^{N}(t ; q)\right) & =\left(\alpha(t) e^{-A(q) t} B(q)\right) \mathrm{d} t .
\end{aligned}
$$

The heat equation solution now is dependent only on time.

\subsubsection{Temporal Discretization}

Ultimately, it is desired to put the equation into a fully time $t$ and depth $\eta$ discretized form in order to integrate with respect to $t$ the value of the alcohol concentration dissipating through the dermal layer at a specific time and skin depth as entries of a matrix. Thus it is necessary to now discretize Equation (2.64) over uniform sub-intervals of time. Let $\tau$ be the length of the sampling interval and $T$ denote the number of intervals. Define a time sub-interval as $\left[t_{j}, t_{j+1}\right)$ for $j=0,1,2, \ldots, T$. Consider the number line for such a time interval below

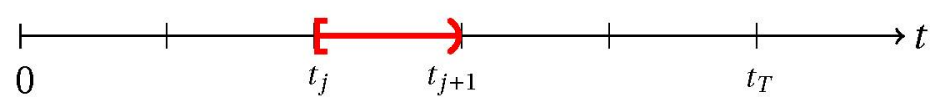


Given that each sub-interval has length $\tau$, the $j^{\text {th }}$ point in time is $t_{j}=j \tau$ in length from $t=0$. Thus the number line is

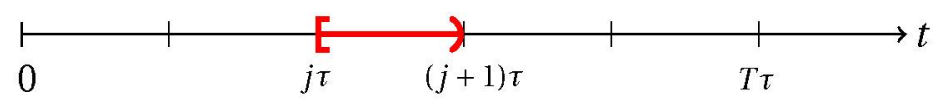

Recall from Equation (2.10) that the input to the system is defined in terms of $t$ as

$$
\alpha(t)=\frac{q_{1}}{q_{2}} \frac{\partial u}{\partial \eta}(1, t)
$$

Let $\psi(t)$ be a test function, defined in the finite element method, be an order one B-spline. Discretize $t$ by uniformly partitioning $[0, T \tau]$ into $T$ parts. Let $\hat{\psi}(t) \in V$ be an $(T+1) \times 1$ vector of ordered order one B-spline equations evaluated at $t$. The $j^{t h}$ entry of $\hat{\psi}(t)$ is $\psi_{j}(t)$ which is the $j^{t h}$ basis spline. Define $\psi_{j}(t)$, for $j=0,1, \ldots, T-1$ as

$$
\psi_{j}(t)= \begin{cases}1 & \text { if } j \tau \leq t<(j+1) \tau \\ 0 & \text { elsewhere }\end{cases}
$$

The $T^{t h}$ index order one B-spline is unique from the others due to how it only includes the right-hand side of the interval $[(T-1) \tau, T \tau]$. It is defined below

$$
\psi_{T}(t)= \begin{cases}1 & \text { if } t=T \tau \\ 0 & \text { elsewhere }\end{cases}
$$


The graph of the set of ordered order one B-spline bases when $T=5$ is shown in Figure 2.15. Note that, in the order one case, the B-splines are simply indicator functions.

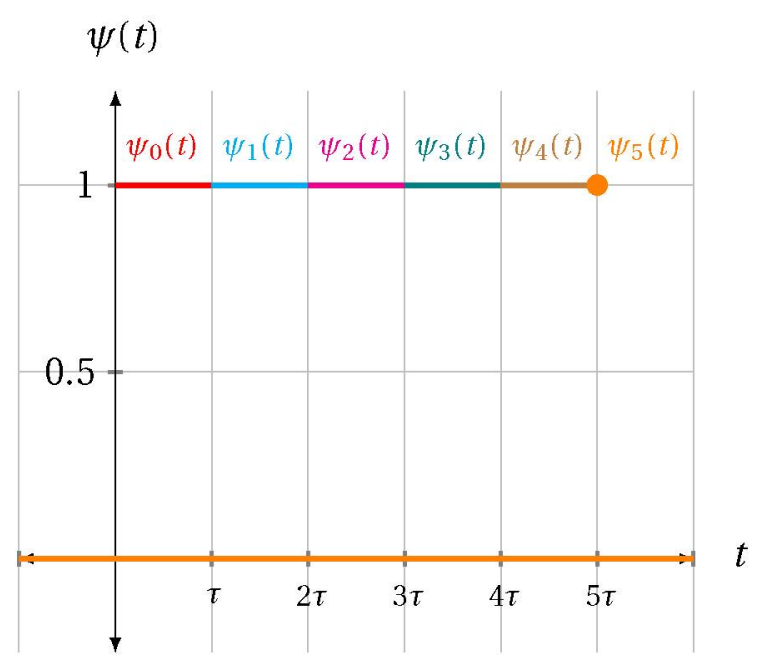

Figure 2.15: Example of order one B-Splines, $T=5$

Let $\hat{\alpha}(q) \in V$ be an $(T+1) \times 1$ vector of $\alpha$-valued points sampled at each respective $j^{t h}$ interval of time $t$. The $j^{t h}$ entry of $\hat{\alpha}(q)$ is denoted as $\alpha_{j}^{T}(q)$ which represents the function value of $\alpha(t)$ at the $j^{t h}$ spline knot. Observe that $\alpha(t)$ can be approximated as an inner product between $\hat{\alpha}(q)$ and $\hat{\psi}(t)$ by defining

$$
\alpha^{T}(t ; q)=\langle\hat{\alpha}(q), \hat{\psi}(t)\rangle_{V^{*}}=\sum_{j=0}^{T} \alpha_{j}^{T}(q) \psi_{j}(t) .
$$

Let $\tau \in \mathbb{R}$ be sufficiently small enough such that $\alpha(t)$ input of alcohol is assumed to stay constant over each sub-interval of time $\left[t_{j}, t_{j+1}\right)$. When this 
condition is met, then we define

$$
\alpha_{j}=\alpha^{T}(t ; q)=\alpha_{j}^{T}(q)
$$

for all $t \in\left[t_{j}, t_{j+1}\right)$. This approximates $\alpha(t)$ by the input value $\alpha_{j}$ at the start of a given sub-interval of time $\left[t_{j}, t_{j+1}\right)$. Now we can integrate the right-hand side of Equation (2.64) on $\left[t_{j}, t_{j+1}\right)=[j \tau,(j+1) \tau)$

$$
\begin{aligned}
\int_{t_{j}}^{t_{j+1}} \mathrm{~d}\left(e^{-A(q) s} \hat{u}^{N}(s ; q)\right) & =\int_{t_{j}}^{t_{j+1}}\left(\alpha(t) e^{-A(q) s} B(q)\right) \mathrm{d} s \\
\int_{t_{j}}^{t_{j+1}} \mathrm{~d}\left(e^{-A(q) s} \hat{u}^{N}(s ; q)\right) & =\int_{t_{j}}^{t_{j+1}}\left(\alpha_{j} e^{-A(q) s} B(q)\right) \mathrm{d} s \\
\int_{j \tau}^{(j+1) \tau} \mathrm{d}\left(e^{-A(q) s} \hat{u}^{N}(s ; q)\right) & =\int_{j \tau}^{(j+1) \tau}\left(\alpha_{j} e^{-A(q) s} B(q)\right) \mathrm{d} s \\
\int_{j \tau}^{(j+1) \tau} \mathrm{d}\left(e^{-A(q) s} \hat{u}^{N}(s ; q)\right) & =\left[\int_{j \tau}^{(j+1) \tau} e^{-A(q) s} \mathrm{~d} s\right] \cdot \alpha_{j} B(q) .
\end{aligned}
$$

Note that $A(q)=M^{-1}\left(-L-q_{1} K\right)$ is always invertible for $1 \leq N<\infty$ since the matrix is positive-definite and symmetric. Integrating while using this observation gives us

$$
\begin{aligned}
{\left.\left[e^{-A(q) s} \hat{u}^{N}(s ; q)\right]\right|_{j \tau} ^{(j+1) \tau} } & =\left.\left[e^{-A(q) s}\left(-A(q)^{-1}\right)\right]\right|_{j \tau} ^{(j+1) \tau} \cdot \alpha_{j} B(q) \\
& =-\left.\left[e^{-A(q) s}\right]\right|_{j \tau} ^{(j+1) \tau} \cdot \alpha_{j} A(q)^{-1} B(q)
\end{aligned}
$$


First, we rewrite the left-hand side of Equation (2.69)

$$
\begin{aligned}
& {\left.\left[e^{-A(q) s} \hat{u}^{N}(s ; q)\right]\right|_{j \tau} ^{(j+1) \tau}} \\
& =e^{-A(q)(j+1) \tau} \hat{u}^{N}((j+1) \tau ; q)-e^{-A(q) j \tau} \hat{u}^{N}(j \tau ; q) \\
& =e^{-A(q) j \tau} \cdot e^{-A(q) \tau} \hat{u}^{N}((j+1) \tau ; q)-e^{-A(q) j \tau} \hat{u}^{N}(j \tau ; q) \\
& =e^{-A(q) j \tau} \cdot\left[e^{-A(q) \tau} \hat{u}^{N}((j+1) \tau ; q)-\hat{u}^{N}(j \tau ; q)\right] .
\end{aligned}
$$

Next, we rewrite the right-hand side of Equation (2.69)

$$
\begin{aligned}
& -\left.\left[e^{-A(q) s}\right]\right|_{j \tau} ^{(j+1) \tau} \cdot \alpha_{j} A(q)^{-1} B(q) \\
& =-\left[e^{-A(q)(j+1) \tau}-e^{-A(q) j \tau}\right] \alpha_{j} A(q)^{-1} B(q) \\
& =-\left[e^{-A(q) j \tau} \cdot e^{-A(q) \tau}-e^{-A(q) j \tau}\right] \alpha_{j} A(q)^{-1} B(q) \\
& =-e^{-A(q) j \tau} \cdot\left[e^{-A(q) \tau}-I_{N+1}\right] \alpha_{j} A(q)^{-1} B(q) \\
& =e^{-A(q) j \tau} \cdot\left[I_{N+1}-e^{-A(q) \tau}\right] \alpha_{j} A(q)^{-1} B(q) .
\end{aligned}
$$

Observe that both Equation (2.70) and Equation (2.71) results have a matrix factor of $e^{-A(q) j \tau}$ in common. This factor is invertible, because it's the exponential of a square matrix. Multiply both sides by $e^{A(q) j \tau}$ and putting the rewritten left-hand and right-hand sides back together gives us

$$
\begin{aligned}
e^{-A(q) \tau} \hat{u}^{N}((j+1) \tau ; q) & -\hat{u}^{N}(j \tau ; q) \\
& =\left[I_{N+1}-e^{-A(q) \tau}\right] \alpha_{j} A(q)^{-1} B(q) .
\end{aligned}
$$


Isolating $\hat{u}^{N}((j+1) \tau ; q)$ yields

$$
\begin{aligned}
e^{-A(q) \tau} \hat{u}^{N}((j+1) \tau ; q) & =\hat{u}^{N}(j \tau ; q)+\left[I_{N+1}-e^{-A(q) \tau}\right] \alpha_{j} A(q)^{-1} B(q) \\
\hat{u}^{N}((j+1) \tau ; q) & =e^{A(q) \tau} \cdot \hat{u}^{N}(j \tau ; q)+e^{A(q) \tau} \cdot\left[I_{N+1}-e^{-A(q) \tau}\right] \alpha_{j} A(q)^{-1} B(q) \\
\hat{u}^{N}((j+1) \tau ; q) & =e^{A(q) \tau} \hat{u}^{N}(j \tau ; q)+\left[e^{A(q) \tau}-I_{N+1}\right] \alpha_{j} A(q)^{-1} B(q) .
\end{aligned}
$$

Now $\hat{u}^{N}((j+1) \tau ; q)$ is only dependent upon the number of time sub-intervals $T$, the number of levels of discretization of skin depth $N$, and the parameters $q=\left\langle q_{1}, q_{2}\right\rangle$. Define

$$
\hat{A}(q)=e^{A(q) \tau}
$$

and

$$
\hat{B}(q)=\left[e^{A(q) \tau}-I_{N}\right] A(q)^{-1} B(q) .
$$

Substituting these results into Equation (2.73) gives

$$
\begin{aligned}
\hat{u}^{N}((j+1) \tau ; q) & =e^{A(q) \tau} \hat{u}^{N}(j \tau ; q)+\alpha_{j}\left[e^{A(q) \tau}-I_{N+1}\right] A(q)^{-1} B(q) \\
\hat{u}^{N}((j+1) \tau ; q) & =\hat{A}(q) \hat{u}^{N}(j \tau ; q)+\alpha_{j} \hat{B}(q) \\
\hat{u}^{N}\left(t_{j+1} ; q\right) & =\hat{A}(q) \hat{u}^{N}\left(t_{j} ; q\right)+\alpha_{j} \hat{B}(q) .
\end{aligned}
$$

The resulting Equation (2.76) is a recursive vector equation for $u(\eta, t ; q)$, which is discretized over normalized skin depth in $[0,1]$ and defined for a time interval $\left[t_{j}, t_{j+1}\right)$ of length $\tau$. 
Define $U^{N, T}(q)$ as the $(N+1) \times(T+1)$ matrix with $\hat{u}^{N}\left(t_{j} ; q\right)$ as columns for $j=0,1, \ldots, T$ that discretizes the time $t$ into $T$ intervals over the columns and discretizes the skin depth $\eta$ into $N$ levels over the rows. The set of recursion vectors at each $t_{j}$ serves as a column space for the vector space defined by $U^{N, T}(q)$

The recursion with respect to the model in Equation (2.76) can be solved for an explicit solution. We do this inductively. Consider the case when $j=2$ :

$$
\begin{aligned}
\hat{u}^{N}\left(t_{3} ; q\right) & =\hat{A}(q) \hat{u}^{N}\left(t_{2} ; q\right)+\alpha_{2} \hat{B}(q) \\
& =\hat{A}(q)\left(\hat{A}(q) \hat{u}^{N}\left(t_{1} ; q\right)+\alpha_{1} \hat{B}(q)\right)+\alpha_{2} \hat{B}(q) \\
& =\hat{A}(q)\left(\hat{A}(q)\left(\hat{A}(q) \hat{u}^{N}\left(t_{0} ; q\right)+\alpha_{0} \hat{B}(q)\right)+\alpha_{1} \hat{B}(q)\right)+\alpha_{2} \hat{B}(q) \\
& =(\hat{A}(q))^{3} \hat{u}^{N}\left(t_{0} ; q\right)+\left(\alpha_{0}(\hat{A}(q))^{2}+\alpha_{1} \hat{A}(q)+\alpha_{2}\right) \hat{B}(q) \\
& =(\hat{A}(q))^{3} \hat{u}^{N}\left(t_{0} ; q\right)+\sum_{k=1}^{3}\left(\alpha_{k-1}(\hat{A}(q))^{3-k}\right) \hat{B}(q)
\end{aligned}
$$

Assume the pattern holds for the $j=m-1$ case. Then,

$$
\hat{u}^{N}\left(t_{m} ; q\right)=(\hat{A}(q))^{m} \hat{u}^{N}\left(t_{0} ; q\right)+\sum_{k=1}^{m}\left(\alpha_{k-1}(\hat{A}(q))^{m-k}\right) \hat{B}(q)
$$


Consider the case when $j=m$ :

$$
\begin{aligned}
\hat{u}^{N}\left(t_{m+1} ; q\right) & =\hat{A}(q) \hat{u}^{N}\left(t_{m} ; q\right)+\alpha_{m} \hat{B}(q) \\
& =\hat{A}(q)\left[(\hat{A}(q))^{m} \hat{u}^{N}\left(t_{0} ; q\right)+\sum_{k=1}^{m}\left(\alpha_{k-1}(\hat{A}(q))^{m-k}\right) \hat{B}(q)\right]+\alpha_{m} \hat{B}(q) \\
& =(\hat{A}(q))^{m+1} \hat{u}^{N}\left(t_{0} ; q\right)+\sum_{k=1}^{m}\left(\alpha_{k-1}(\hat{A}(q))^{(m+1)-k}\right) \hat{B}(q)+\alpha_{m} \hat{B}(q) .
\end{aligned}
$$

Since

$$
(\hat{A}(q))^{(m+1)-(m+1)}=(\hat{A}(q))^{0}=I_{N+1},
$$

it follows that

$$
\begin{aligned}
\alpha_{m} \hat{B}(q) & =\alpha_{(m+1)-1} I_{N+1} \hat{B}(q) \\
& =\alpha_{(m+1)-1}(\hat{A}(q))^{(m+1)-(m+1)} \hat{B}(q)
\end{aligned}
$$

Thus, Equation (2.79) simplifies to

$$
\begin{aligned}
& (\hat{A}(q))^{m+1} \hat{u}^{N}\left(t_{0} ; q\right)+\sum_{k=1}^{m}\left(\alpha_{k-1}(\hat{A}(q))^{(m+1)-k}\right) \hat{B}(q)+\alpha_{m} \hat{B}(q) \\
& =(\hat{A}(q))^{m+1} \hat{u}^{N}\left(t_{0} ; q\right)+\sum_{k=1}^{m}\left(\alpha_{k-1}(\hat{A}(q))^{(m+1)-k}\right) \hat{B}(q) \\
& +\alpha_{(m+1)-1}(\hat{A}(q))^{(m+1)-(m+1)} \hat{B}(q) \\
& =(\hat{A}(q))^{m+1} \hat{u}^{N}\left(t_{0} ; q\right)+\sum_{k=1}^{m+1}\left(\alpha_{k-1}(\hat{A}(q))^{(m+1)-k}\right) \hat{B}(q) .
\end{aligned}
$$


Therefore, we have proved by mathematical induction that the explicit equation shown in Equation (2.81), defined for any $j=1,2, \ldots, T$, is given by

$$
\hat{u}^{N}\left(t_{j} ; q\right)=(\hat{A}(q))^{j} \hat{u}^{N}\left(t_{0} ; q\right)+\sum_{k=1}^{j}\left(\alpha_{k-1}(\hat{A}(q))^{j-k}\right) \hat{B}(q)
$$

Recall the boundary condition, $u(\eta, 0)=0$ from, from Equation (2.8). Thus since $t_{0}=0$ we have $\hat{u}^{N}\left(t_{0} ; q\right)=\hat{u}^{N}(0 ; q)=\overrightarrow{0}$. This simplifies Equation (2.82) as

$$
\hat{u}^{N}\left(t_{j} ; q\right)=\sum_{k=1}^{j}\left(\alpha_{k-1}(\hat{A}(q))^{j-k}\right) \hat{B}(q)
$$

Therefore, the alcohol concentration matrix, described as a column space, is

$$
\begin{aligned}
U^{N, T}(q) & =\left[\hat{u}^{N}\left(t_{0} ; q\right),\left\{\hat{u}^{N}\left(t_{j} ; q\right)\right\}_{j=1}^{T}\right] \\
& =\left[\overrightarrow{0},\left\{\sum_{k=1}^{j}\left(\alpha_{k-1}(\hat{A}(q))^{j-k}\right) \hat{B}(q)\right\}_{j=1}^{T}\right] .
\end{aligned}
$$

An entry of the matrix $\left(U^{N, T}(q)\right)_{i, j}$ is the alcohol concentration at depth $\eta_{i}=$ $\frac{i}{N}$ and time $t_{j}=j \tau$ for $i=0, \ldots, N$ and $j=0, \ldots, T$. Observe that this formulation aligns with the physical system rather well. It logically follows that the alcohol concentration measurement is dependent upon the sequence of inputs at various points in time and the parameters of diffusivity determined by the composition of the individual's dermal tissue. This approximation satisfies item (iii) of the finite element method discussed at the beginning of section two. 


\subsection{Transdermal Alcohol Concentration}

In order to estimate the joint mixing distribution for the parameters of diffusivity in Chapter 3, we need to approximate the transdermal alcohol concentration for $P$ individuals of a population. The transdermal level, defined as $\eta=0$, is the first row of the $U^{N, T}(q)$ matrix in Equation (2.83).

The matrix can be used to estimate other dermal levels as well. Note however that it may require the linear interpolation between the two nearest discretized points of the $[0,1]$ interval when the desired $\eta$ depth is not equal to one of the $\frac{i}{N}$ points for each $i \in\{0,1, \ldots, N\}$. Define a function $\hat{c}(\eta)$ for this purpose which is a $1 \times(N+1)$ vector which has zeros in all entries except the two entries nearest to the $\eta$ input. These two entries have weights based on the discretized points respective distance from the specified $\eta$ depth. For the purposes of this thesis, we only consider $\hat{c}(0)$, which corresponds to the transdermal (skin) level. Therefore, define $y$ as the $1 \times(T+1)$ vector

$$
y(q)=\hat{c}(0) U^{N, T}(q)
$$

whose entries are the approximated transdermal alcohol concentration measurements from time $t_{0}=0$ to $t_{T}=T \cdot \tau$ for an individual with their personal 
parameters of diffusivity given by $q$. See Algorithm 2 in Appendix B for the pseudocode implementation of $y(q)$. 


\subsection{Error in Measurements}

The explicit equation provided in Equation (3.84)

$$
y(q)=\hat{c}(0) U^{N, T}(q)
$$

is the result of the discretization process via the finite element method which provides an approximation for the transdermal alcohol concentration. However, it does not account for error in the measurements caused by biological factors such as a layer of moisture or oil from dermal glands, or environmental conditions such as air temperature. These types of influencing elements can typically be assumed to be normally distributed and they should only cause small perturbations (above or below) to the given measurements.

Let $\epsilon$ be the $1 \times(T+1)$ vector of error values with entries sampled from a normal distribution with mean 0 and standard deviation $\sigma$. Let $y(q)$ be the approximation of transdermal alcohol concentration from Equation (3.84) in the previous section. If $y_{\epsilon}(q)$ takes the $y(q)$ vector while also accounting for the aforementioned error variations, then

$$
y_{e}(q)=y(q)+\epsilon, \quad \epsilon \sim \mathscr{N}\left(0, \sigma^{2}\right)
$$

is the $1 \times(T+1)$ vector of these measurements. See Algorithm 3 in Appendix B for the pseudocode implementation of $y_{\varepsilon}(q)$. 


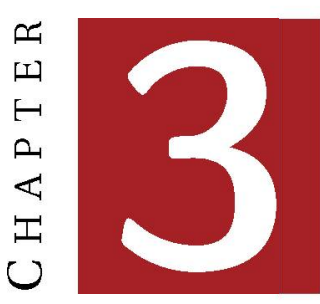

\section{ESTIMATION OF THE JoInT MiXing}

\section{DISTRIBUTION}

In Chapter 2, we chose a heat equation with only one spacial depth dimension to model the transfer of alcohol particles diffusing outward through the skin from a region of high concentration in the blood enclosed in the tissue of the dermal layer to a region of low concentration in the atmosphere. The discretization of this equation into a vector of transdermal alcohol concentrations, as in Equation (4.85)

$$
y_{\epsilon}(q)=y(q)+\epsilon, \quad \epsilon \sim \mathcal{N}\left(0, \sigma^{2}\right)
$$


isolates the parameters in the vector $q=\left\langle q_{1}, q_{2}\right\rangle$. Recall that these parameters correlate to an individual's rate of alcohol diffusion through the dermal tissue while accounting for bio-irregularity.

At this point, it is important to understand that the problem of estimating transdermal alcohol concentration hinges upon knowing the drinking episode schedule of an individual and the parameters of diffusivity. Fortunately, any simple function can be chosen to model the drinking episode. The parameters, $q_{1}$ and $q_{2}$, which are assumed to be independent and distributed with an unknown common joint mixture distribution $F$, can be approximated via maximum likelihood estimation. When the distribution function is known, first derivative tests or similar techniques can usually be used to estimate the parameter values. These methods are therefore unavailable in this instance. However, we outline in this chapter a technique to overcome this problem through the use of nonparametric maximum likelihood techniques. We provide a detailed description in Chapter 4 how the nonparametric maximum likelihood estimation of $F$ can be solved computationally via an advanced algorithm called the nonparametric adapted grid (NPAG) algorithm. See Algorithm 4 in Appendix B. 


\subsection{Maximum Likelihood Estimation}

Maximum likelihood (see Definition A.1.7) is the mathematical process of computing an estimator for a parameter with the highest probability of maximizing the likelihood function. In this problem, the cumulative joint mixture distribution function $F$ is the parameter being estimated, and not $q_{1}$ and $q_{2}$ directly.

Assume the observed data measurements, $Y^{P}=Y_{1}, Y_{2}, \ldots, Y_{P}$, are independent and identically distributed, with respect to some $\sigma$-finite measure. Each $Y_{i}$ measurement, from $i^{t h}$ individual, has the probability density function $p_{i}\left(Y_{i} \mid q\right)$ where $q=\left\langle q_{1}, q_{2}\right\rangle$ is the vector of diffusivity parameters. Then, the log-likelihood function (see Definition A.1.4 and [21]) for this problem is defined as

$$
\begin{aligned}
\ell(F) & =\ell\left(Y_{1}, Y_{2}, \ldots, Y_{P} \mid F\right) \\
& =\log \left(p\left(Y_{1}, Y_{2}, \ldots, Y_{P} \mid F\right)\right) .
\end{aligned}
$$

Note that the log-likelihood function, denoted by $\ell$, is used here instead of the likelihood function, denoted by $L$, because of the logarithm property that the $\log$ of the product of terms becomes the sum of the log of terms. This provides easier formulations as shown in the next calculation. There are independent 
measurements so the conditional pdf can split into a product of conditional pdf's

$$
\begin{aligned}
\log \left(p\left(Y_{1}, Y_{2}, \ldots, Y_{P} \mid F\right)\right) & =\log \left(\prod_{i=1}^{P} p_{i}\left(Y_{i} \mid F\right)\right) \\
& =\sum_{i=1}^{P} \log \left(p_{i}\left(Y_{i} \mid F\right)\right) .
\end{aligned}
$$

The mixture density function (see Definition A.3.20) corresponding to the joint mixture distribution $F$, where the probability measure $F$ is over the parameter space $\Omega$, is defined as

$$
p_{i}\left(Y_{i} \mid F\right)=\int_{\Omega} p_{i}\left(Y_{i} \mid q\right) \mathrm{d} F(q) .
$$

Combining Equation (1.4) with Equation (1.5) gives

$$
\ell(F)=\sum_{i=1}^{P} \log \left(\int_{\Omega} p_{i}\left(Y_{i} \mid q\right) \mathrm{d} F(q)\right) .
$$

The maximum likelihood estimator is calculated by way of comparing of possible parameter values. Let $\mathscr{F}$ be the family of all probability distributions over the parameter space $\Omega$. Take each $F \in \mathscr{F}$, and then compare the likelihood function at every combination of parameter point pairs $q=\left\langle q_{1}, q_{2}\right\rangle$. Cassela and Berger [1], on page 290, and Schumitzky [21] observe that when

$$
L\left(F^{*}\right) \geq L(F)
$$


for all $F \in \mathscr{F}$, then the maximum likelihood estimator $F^{M L}$ is more likely to be $F^{*}$ than any other $F$. In other words, the maximum likelihood estimator of $F$ (see Definition A.1.7) is

$$
F^{M L}=\operatorname{argmax}\{\ell(F): F \in \mathscr{F}\}
$$

The maximum likelihood estimator, as stated, could be any one of infinitely many possible joint mixture distributions for the parameter in the space of all probability density functions. Thus it would be impossible to find and verify the distribution exhaustively. The theory for a procedure to overcome this challenge is presented in the next section. 


\subsection{Reduction to Finite Support}

Research published by Lindsay [13] using convexity theory offers a reliable method to reduce the problem to a more reasonable subclass of possibilities. Lindsay provides a geometric approach to likelihood. A clever application of the Carathéodory Theorem (see Theorem A.3.19) is utilized since both the space of probability density functions and its convex hull are finitedimensional and measurable. Each possible $F$, defined on the parameter space $\Omega$, is in $\mathscr{F}$ and thus the maximum likelihood estimate $F^{M L}$ is in the space of only discrete distributions with $K$ support points, denoted $\mathscr{F}_{K}$ (where $K$ is no more than the number of observed measurements $P$ ).

Let $\xi_{k}$ be the support points of $F$. Let $\left\{f_{\xi}: \xi \in \Omega\right\}$ be a parametric family of densities with respect to some sigma-finite measure. Let the parameter space $\Omega$ have a sigma field of measurable sets which contains all atomic sets $\{\xi\}$. Let $\mathscr{M}$ be the class of all probability measures on $\Omega$. Lindsay shows that the arbitrary convex combination of elements of the convex hull (see Definition A.3.16) of the likelihood curve $\Gamma=\left\{f_{\xi}: \xi \in \Omega\right\}$ (see Definition A.3.22), defined as $H(\Gamma)=\left\{f_{F}: F \in \mathscr{M}\right.$, such that $F$ has finite support $\}$, can be written as

$$
\sum_{k=1}^{K} \pi_{k} f_{\xi_{k}},
$$


with restrictions that

a) $\xi_{1}, \ldots, \xi_{K}$ are distinct elements of $\Omega$,

b) $\pi_{k}>0$ for all $k$,

c) $\sum_{k=1}^{K} \pi_{k}=1$

See Figure 3.1 for an example how an interior point of the convex hull is a convex combination of no more than three of the four points on the boundary of the convex hull.

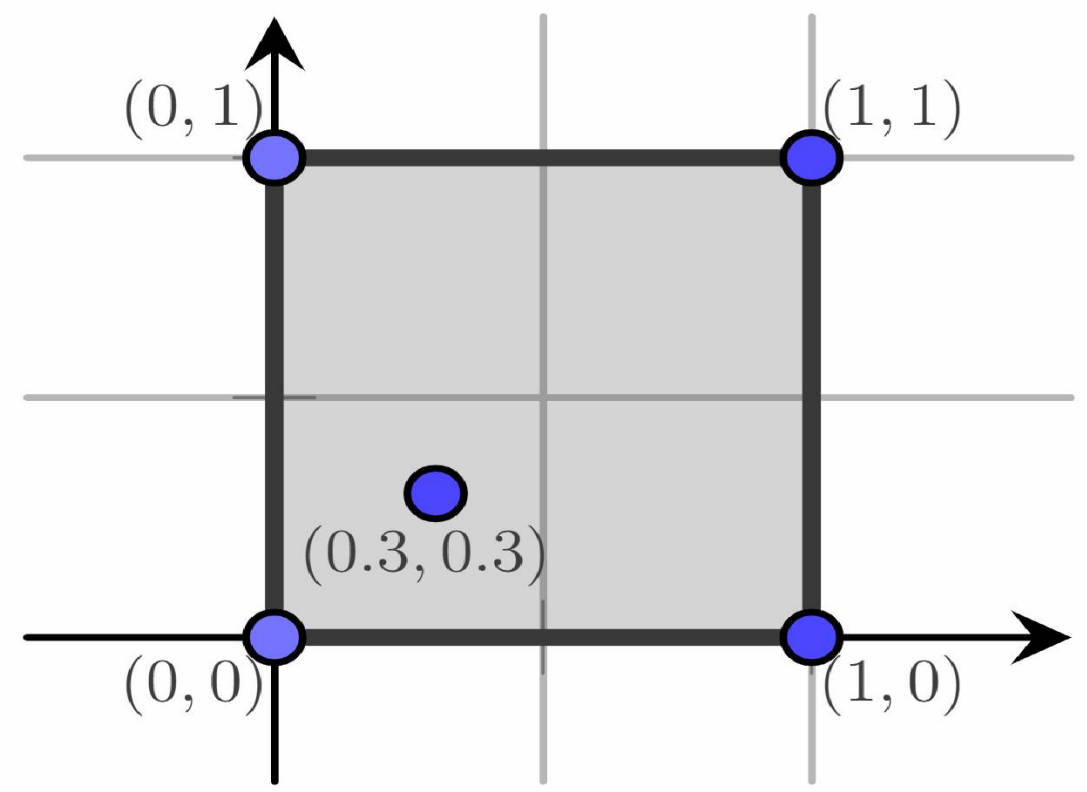

Figure 3.1: Convex Combination Example

Let $\delta_{\xi}(q)=\delta(x-\xi)$ be an atomic density centered at $\xi$, as in Definition A.3.21. In the doctoral dissertation by Dr. Kryshchenko [3], it is observed that 
this atomic density measure gives

$$
\int g(q) \delta_{\xi}(q) \mathrm{d} q=g(\xi)
$$

for any density function $g$. Note that the Heaviside function (see Definition A.3.24) and the atomic density are related by $H^{\prime}=\delta$. Therefore,

$$
H(x)=\int_{-\infty}^{x} \delta(t) \mathrm{d} t
$$

which means the Heaviside function is the cdf of the delta function centered at zero. This is important when considering the Lebesgue integral in Equation (2.10). To clarify the connection even further, observe that

$$
\int_{-\infty}^{\infty} g(x) \mathrm{d} H(x)=\int_{-\infty}^{\infty} g(x) \delta(x-\xi) \mathrm{d} x=g(\xi)
$$

for some density function $g$. Note $F$ is an empirical distribution which can be written as a linear combination of Heaviside functions. Hence, $\mathrm{d} F$ can be written as a convex combination of the form

$$
\mathrm{d} F=\sum_{k=1}^{K} w_{k} \delta_{\xi_{k}}, \quad K \leq P .
$$

In the construction presented by Lindsay together with the specifics of the alcohol biosensor problem, the mixture likelihood vector entries are

$$
f_{F}\left(Y_{i}\right)=\int f_{q}\left(Y_{i}\right) \mathrm{d} F(q), \quad F \in \mathscr{H}
$$


Observe that when Equation (2.12) is compared with Equation (1.5), then it is clear that $f_{F}\left(Y_{i}\right)=p_{i}\left(Y_{i} \mid F\right)$ and $f_{q}\left(Y_{i}\right)=p_{i}\left(Y_{i} \mid q\right)$. Applying this together with Equation (2.10) and Equation (2.11) gives a significant result that

$$
\begin{aligned}
\int f_{q}\left(Y_{i}\right) \mathrm{d} F(q) & =\int p_{i}\left(Y_{i} \mid q\right) \mathrm{d} F(q) \\
& =\int p_{i}\left(Y_{i} \mid q\right) \sum_{k=1}^{K} w_{k} \delta_{\xi_{k}}(q) \mathrm{d} q \\
& =\sum_{k=1}^{K} w_{k} \int p_{i}\left(Y_{i} \mid q\right) \delta_{\xi_{k}}(q) \mathrm{d} q \\
& =\sum_{k=1}^{K} w_{k} p_{i}\left(Y_{i} \mid \xi_{k}\right) .
\end{aligned}
$$

Therefore if $\Omega$ is compact, then using Equation (1.6) together with Equation (2.13) symbolically reduces Equation (1.8) to

$$
\begin{aligned}
F^{M L} & =\operatorname{argmax}\{\ell(F): F \in \mathscr{F}\} \\
& =\operatorname{argmax}\left\{\sum_{i=1}^{P} \log \left(\int_{\Omega} f_{q}\left(Y_{i}\right) \mathrm{d} F(q)\right): F \in \mathscr{F}\right\} \\
& =\operatorname{argmax}\left\{\sum_{i=1}^{P} \log \left(\int_{\Omega} p_{i}\left(Y_{i} \mid q\right) \mathrm{d} F(q)\right): F \in \mathscr{F}\right\} \\
& =\operatorname{argmax}\left\{\sum_{i=1}^{P} \log \left(\sum_{k=1}^{K} w_{k} p_{i}\left(Y_{i} \mid \xi_{k}\right)\right), K \leq P\right\},
\end{aligned}
$$

where all $w_{k}$ weights are nonnegative and sum to one and the $\xi_{k} \in \Omega$ are support points of $F$. It follows that any joint mixture distribution $F \in \mathscr{F}_{K}$, is now 
solely dependent upon the vector of weights and support points defined as

$$
w^{K}=\left(w_{1}, w_{2}, \ldots, w_{K}\right) \in \mathbb{R}^{K}
$$

and

$$
\xi^{K}=\left(\xi_{1}, \xi_{2}, \ldots, \xi_{K}\right) \in \Omega^{K}
$$

respectively. To clarify, each $w_{k}$ is proportional to the probability of its respective point of support; i.e. symbolically $w_{k} \propto \mathbb{P}\left(q=\xi_{k}\right)$. Let

$$
\lambda=\left(\xi^{K}, w^{K}\right)=\left(\xi_{1}, \xi_{2}, \ldots, \xi_{K}, w_{1}, w_{2}, \ldots, w_{K}\right)
$$

be the vector of weights and supports. It is now appropriate to state the question as

$$
\ell(F) \approx \ell(\lambda)=\ell\left(w_{1}, w_{2}, \ldots, w_{K}, \xi_{1}, \xi_{2}, \ldots, \xi_{K}\right)
$$

Estimating the maximum likelihood will require maximizing Equation (2.18). This task is completed computationally via the NPAG algorithm which is given in Appendix B as Algorithm 4. The following chapter provides the details of the execution of the program solution and data results. 


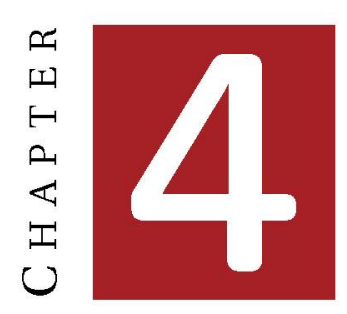

\section{Data Analysis}

Chapter 3 posed the maximum likelihood optimization problem in Equation (1.7) as finding $F^{*}$ such that

$$
L\left(F^{*}\right) \geq L(F)
$$

for all $F \in \mathscr{F}$. In other words, the joint mixing distribution of the parameters of diffusivity has the estimator

$$
F^{M L}=\operatorname{argmax}\{\ell(F): F \in \mathscr{F}\}
$$

A clever application of convex theory reduced the space containing $F^{M L}$ to $\mathscr{F}_{K}$ which is the space of probability distributions with finite support of no more than $K \leq P$ support points where $P$ is the size of the population. The 
optimization problem is restated as

$$
\begin{aligned}
F^{M L} & =\operatorname{argmax}\left\{\sum_{i=1}^{P} \log \left(\sum_{k=1}^{K} w_{k} p_{i}\left(Y_{i} \mid \xi_{k}\right)\right), K \leq P\right\} \\
w_{k} & \geq 0 \\
\sum_{k=1}^{K} w_{k} & =1 .
\end{aligned}
$$

which is now determined by weights $w_{k}$ and support points $\xi_{k}$. A specific $n^{t h}$ combination of these values is represented as

$$
\lambda^{(n)}=\left(\xi_{1}^{(n)}, \xi_{2}^{(n)}, \ldots, \xi_{K}^{(n)}, w_{1}^{(n)}, w_{2}^{(n)}, \ldots, w_{K}^{(n)}\right)
$$

The problem stated in Equation (2.14) is a linear programming optimization problem and by extension a convex optimization problem as well (see Definitions A.3.31 and A.3.42). This follows because an affine (linear) function is also a convex function (see Definition A.3.5). Fortunately, research in an optimization method for problems of this type is ongoing. The method utilized in this paper is the nonparametric adaptive grid (NPAG) algorithm. An article, written in 2019, by a research group on this topic, is heavily referenced for the work that follows in this chapter is titled, "An algorithm for nonparametric estimation of a multivariate mixing distribution with applications to population pharmacokinetics" [25]. 


\subsection{Simulation of Population Data}

We need population data is necessary to execute the NPAG algorithm to estimate the joint mixing distribution of the parameters of diffusivity. Although this thesis only details the role of simulated data, future research will consider data from the drinking episodes of human subjects as well. That empirical human subject data replaces the role of simulated data in the NPAG algorithm. The work of developing a suitable model for simulating transdermal alcohol concentration data for an individual was the subject of Chapter 2. See Algorithms 2 and 3 for the respective implementations. The result at the end of Chapter 2 section 4 provided Equation (A.4.1) below with $\sigma=0.005$.

$$
y_{\epsilon}(q)=y(q)+\epsilon, \quad \epsilon \sim \mathscr{N}\left(0, \sigma^{2}\right)
$$

Recall that the $1 \times(T+1)$ vector of simulated concentration values in Equation (1.7) above relies upon the complete drinking schedule and parameters of diffusivity of the individual (see Equation (2.83)). It is important to note that this research assumes that every individual in the simulated population shares the same drinking schedule. This facilitates the isolation of $q=\left\langle q_{1}, q_{2}\right\rangle$ as the only variable in the experiment. 
Using Equation (1.7), the process of simulating the desired population data is a matter of generating $P$ iterations of $y_{c}(q)$ from $P$ sampled $q$ vectors. These sampled values for $q_{1}$ and $q_{2}$ can be distributed from your choice of probability density functions, $f_{q_{1}}$ and $f_{q_{2}}$ respectively, and they need not necessarily be identically distributed. Please note that these chosen probability distributions and their respective parameters should align with the human biology of dermal diffusion. Collect these sampled values into two $1 \times P$ vectors, $q^{(1)}$ and $q^{(2)}$ respectively and denote the specific values by $q_{k}^{(1)}$ and $q_{k}^{(2)}$ for $k=1, \ldots, P$. Now combine these vectors into a $2 \times P$ matrix defined as

$$
q^{(P)}=\left[\begin{array}{l}
q^{(1)} \\
q^{(2)}
\end{array}\right]
$$

Next generate the $P \times(T+1)$ matrix using these values for $q$ in Equation (1.7).

$$
Y_{\epsilon}\left(q^{(P)}\right)=\left[\begin{array}{c}
y_{\epsilon}\left(q^{(P)}[:, 1]\right) \\
y_{\epsilon}\left(q^{(P)}[:, 2]\right) \\
\vdots \\
y_{\epsilon}\left(q^{(P)}[:, P]\right)
\end{array}\right]
$$

See Algorithm 1 in Appendix B for full details on implementation instructions. The following items share the same variance of $\sigma^{2}=0.01^{2}$ and the means are specified for each distribution. We selected three distinct joint mixing dis- 
tributions for $F$ to generate the population data for $P=100$ individuals. Note these random variables are independent. The three pairs of probability density functions are:

Pair 1: $\quad q_{1} \sim \mathcal{N}\left(1, \sigma^{2}\right)$

$$
q_{2} \sim \mathcal{N}\left(1, \sigma^{2}\right)
$$

Pair 2: $\quad q_{1} \sim 0.7 \mathcal{N}\left(0.7, \sigma^{2}\right)+0.3 \mathcal{N}\left(1, \sigma^{2}\right)$

$$
q_{2} \sim \mathcal{N}\left(1, \sigma^{2}\right)
$$

Pair 3: $\quad q_{1} \sim 0.7 \mathscr{N}\left(0.7, \sigma^{2}\right)+0.3 \mathscr{N}\left(1, \sigma^{2}\right)$

$$
q_{2} \sim 0.3 \mathscr{N}\left(0.7, \sigma^{2}\right)+0.7 \mathscr{N}\left(1, \sigma^{2}\right)
$$

In the first pair, we sampled the elements of the $q^{(1)}$ and $q^{(2)}$ vectors from a normal distribution with mean 1 and variance $0.01^{2}$, because biological problems tend to often have normally distributed values. See Figure 4.1. The plot of the TAC reveals only small variations between individuals in the population. This is expected since both parameters share the same distribution and the TAC values are calculated using identical drinking schedules.

In the second pair, we sampled the elements of the $q^{(1)}$ vector from a bimodal mixing distribution which is composed of two normal distributions. Seventy percent of the distribution is sampled from a normal distribution with 


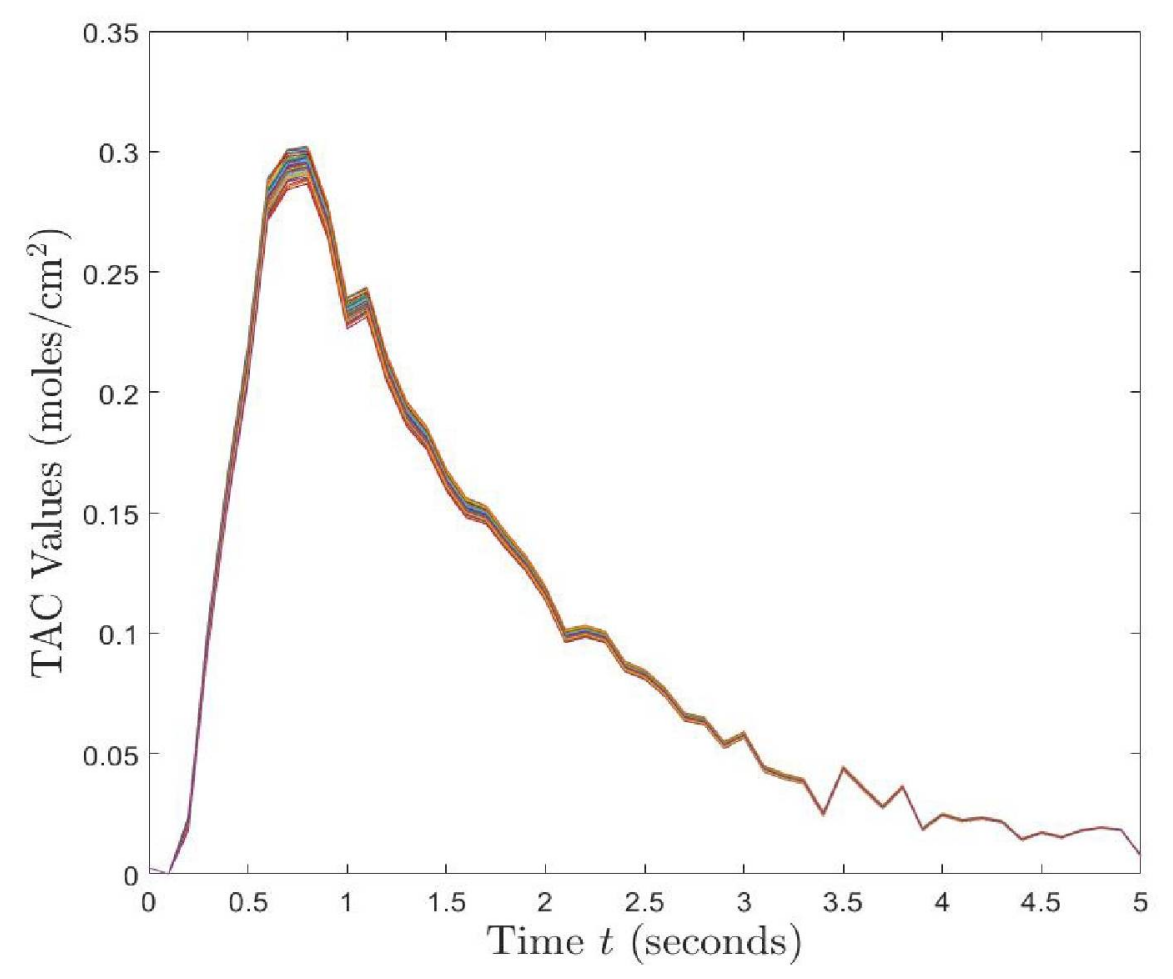

Figure 4.1: Plot of Simulated TAC for a Population of $P=100$ given $q_{1} \sim$ $\mathscr{N}\left(1, \sigma^{2}\right)$ and $q_{2} \sim \mathscr{N}\left(1, \sigma^{2}\right)$

mean 1 and variance $0.01^{2}$, and the other thirty percent of the distribution is sampled from a normal distribution with mean 0.7 and variance $0.01^{2}$. We sampled the elements of the $q^{(2)}$ vector from a normal distribution with mean 1 and variance $0.01^{2}$. We chose a bimodal mixing distribution for $q_{1}$ values, because there may exist two groups with distinct skin compositions. Recall from Equations (2.7) and (2.9) that $q_{1}$ is specifically related to the rate of diffusion through the dermal layer. See Figure 4.2. Note that the time units in the 
model are flexible and replacing seconds with another unit of time leaves the graphs unaffected.

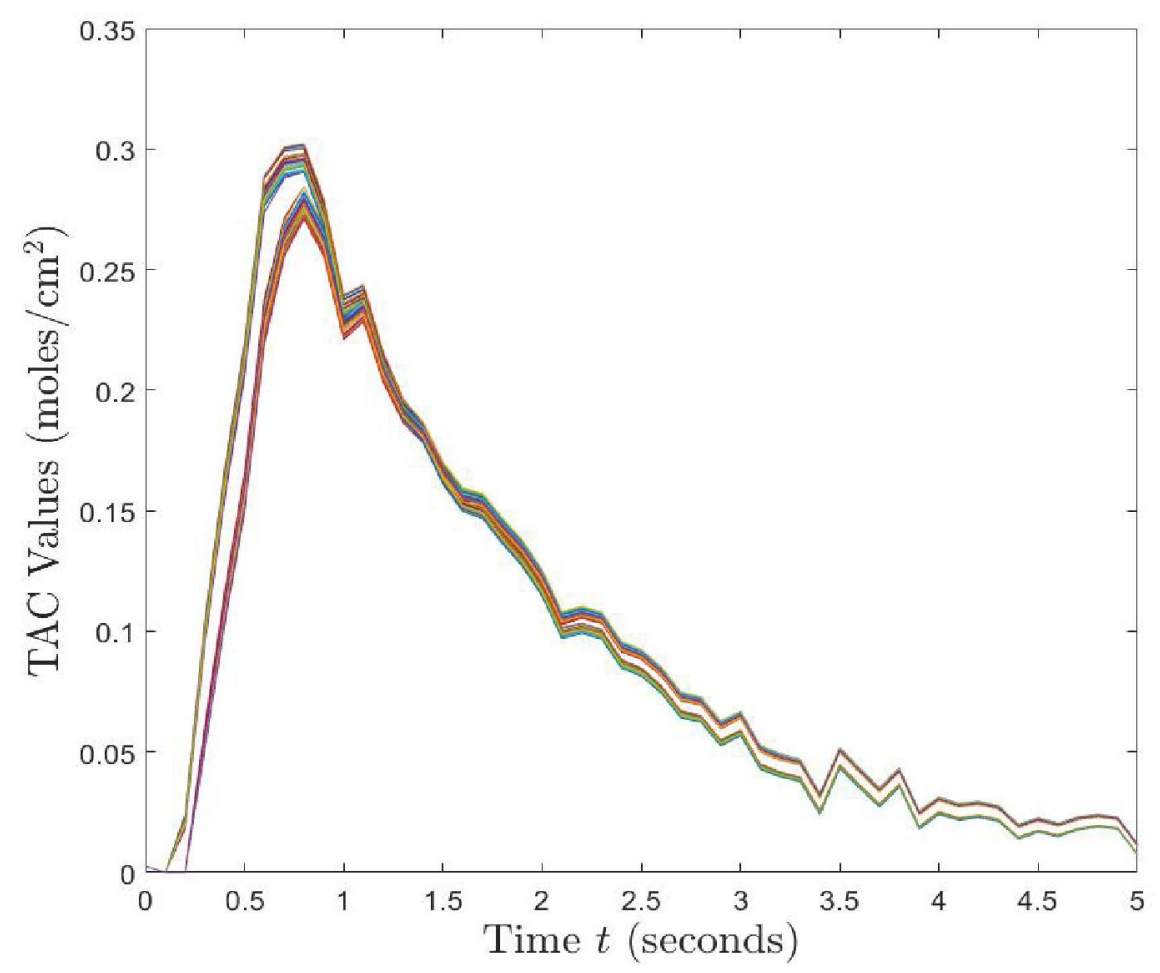

Figure 4.2: Plot of Simulated TAC for a Population of $P=100$ given $q_{1} \sim$ $0.7 \mathscr{N}\left(0.7, \sigma^{2}\right)+0.3 \mathscr{N}\left(1, \sigma^{2}\right)$ and $q_{2} \sim \mathscr{N}\left(1, \sigma^{2}\right)$

The split of the two populations is observed as two disparate bands in the plot of the TAC. There is still only a small variation of TAC values within a single band since they are calculated using identical drinking schedules and any given curve within that band will have diffusion parameters which are either all sampled near the mean of 1 or the mean of 0.7 . 
In the last pair, we sampled the elements of the $q^{(1)}$ vector from a bimodal mixing distribution which is composed of two normal distributions. Seventy percent of the distribution is sampled from a normal distribution with mean 1 and variance $0.01^{2}$, and the other thirty percent of the distribution is sampled from a normal distribution with mean 0.7 and variance $0.01^{2}$. We sampled the elements of the $q^{(2)}$ vector from a bimodal mixing distribution which is composed of two normal distributions. Thirty percent of the distribution is sampled from a normal distribution with mean 1 and variance $0.01^{2}$. and the other seventy percent of the distribution is sampled from a normal distribution with mean 0.7 and variance $0.01^{2}$. We chose a bimodal distribution for $q_{1}$ values for the same reason as in pair two. We chose a bimodal distribution for $q_{2}$ because biological differences exist between how two different body types metabolize alcohol. Note from Equation (2.10) that the $q_{2}$ parameter is directly linked to the input of alcohol from the blood stream into the dermal tissue. See Figure 4.3. 


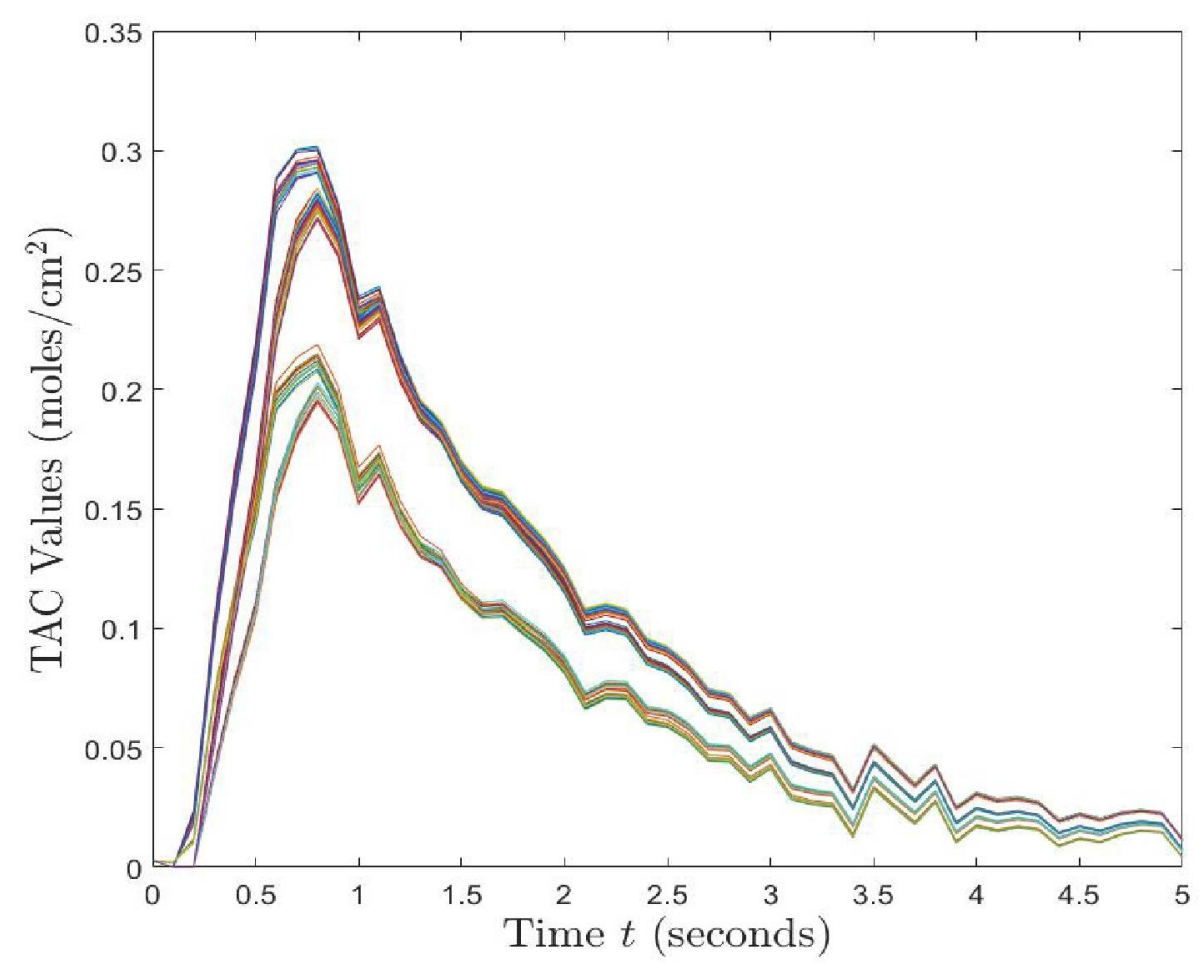

Figure 4.3: Plot of Simulated TAC for a Population of $P=100$ given $q_{1} \sim$ $0.7 \mathscr{N}\left(0.7, \sigma^{2}\right)+0.3 \mathcal{N}\left(1, \sigma^{2}\right)$ and $q_{2} \sim 0.3 \mathscr{N}\left(0.7, \sigma^{2}\right)+0.7 \mathscr{N}\left(1, \sigma^{2}\right)$

The plot of the TAC shows the split of the populations into four bands. Each of the two bands in part two, from skin composition differences, are now further divided into two additional bands due to the split of the two body type populations. As in the previous two pairs, only small variations of TAC values exist within each of the four bands since they are calculated using identical drinking schedules and any curve within a given band will all be sampled near 
one of the four possible mode pairings. 


\subsection{Nonparametric Adapted Grid Algorithm}

The nonparametric adaptive grid (NPAG) algorithm takes observed population data and executes an iterative optimization process over a specified initial grid of support in order to calculate the maximum likelihood estimator for an unknown joint mixing distribution. The "nonparametric" portion of the name derives from the distribution being unknown. "Adaptive grid" refers to the components of the algorithm where the grid of support points is iteratively adjusted to coalesce around the support points of highest probability. A brief summary of the major details of the algorithm follows. Please see Algorithm 4 for the full pseudocode implementation.

\subsubsection{Primal-Dual Interior-Point Method}

The primal-dual interior-point (PDIP) method is essential to the execution of the NPAG algorithm. An outline of the method used in this thesis follows. For a more thorough treatment of this topic which includes clear examples of implementations under various conditions read, "Primal-Dual Interior-Point Methods", written by Dr. Stephen Wright [24]. The previously referenced 2019 paper by the research group [25] has a well explained breakdown of this PDIP 
method in the appendices of the article which is more specific to this type of problem.

The primal problem (see Definitions A.3.25, A.3.31, and A.3.42) is the classical optimization formulation for a system solvable using the Lagrangian (see Definition A.3.34) on the system in Equations (0.3), (0.4), and (0.5). It is important to first define the $P \times K$ matrix of the probabilities of observed data at each point of support as

$$
\Psi\left(Y_{\epsilon}, \xi\right)=\left[\begin{array}{cccc}
p\left(Y_{1} \mid \xi_{1}\right) & p\left(Y_{1} \mid \xi_{2}\right) & \cdots & p\left(Y_{1} \mid \xi_{K}\right) \\
p\left(Y_{2} \mid \xi_{1}\right) & p\left(Y_{2} \mid \xi_{2}\right) & \cdots & p\left(Y_{2} \mid \xi_{K}\right) \\
\vdots & \vdots & \ddots & \vdots \\
p\left(Y_{P} \mid \xi_{1}\right) & p\left(Y_{P} \mid \xi_{2}\right) & \cdots & p\left(Y_{P} \mid \xi_{K}\right)
\end{array}\right] .
$$

This is implemented as the $\Psi$ algorithm (see Algorithm 5). Define the function $\Phi: \mathbb{R}^{P} \rightarrow \mathbb{R} \cup\{+\infty\}$ as

$$
\Phi(z)= \begin{cases}-\log \left(\prod_{i=1}^{P} z[i]\right) & \text { for } z>0 \\ \infty & \text { otherwise }\end{cases}
$$

Note that $z>0$ notation denotes the vector $z$ has strictly positive entries.

Let $\Lambda$ be the $P \times P$ diagonal matrix of Lagrange multipliers, $S$ be the $P \times P$ diagonal matrix of dual slack values, and $e_{P}$ be the $P \times 1$ vector with all entries 
equal to one. Now define the primal problem as

$$
\begin{aligned}
& \mathscr{P}: \text { minimize } \Phi\left(\Psi w^{K}\right) \text {, subject to } \\
& \Lambda \Psi w^{K}=e_{P} \\
& \sum_{k=1}^{K} w_{k}=1 \\
& w^{K} \geq 0
\end{aligned}
$$

Observe that Equation (2.12) is the objective function, Equation (2.13) and Equation (2.14) are the equality constraint functions, and the weights in Equation (2.15) are the inequality constraint functions.

How does this relate to Equation (0.3) in the original formulation? Recall that $w^{K}$ is the $K \times 1$ vector of weights where $w_{k}$ is proportional to the probability of the $k^{\text {th }}$ point of support. Now observe that taking the log of the inner product of the $i^{t h} 1 \times K$ row vector of $\Psi$ with the $K \times 1$ vector of weights $w^{K}$ results in one of the $P$ sums in Equation (0.3). Hence, this gives

$$
\Phi\left(\Psi w^{K}\right)=-F_{\text {estimate }}^{M L}
$$

In addition, notice that the previous maximum likelihood optimization problem in Equation (0.3) has been shifted to a minimization problem by negating the log-likelihood. 
The Fenchel-Rockafellar dual problem (see Definitions A.3.36 and A.3.43) is the counterpart to the convex primal problem which maximizes over the weights and includes a $K \times 1$ slack vector $s$ in the conditions. Define the dual problem to $\mathscr{P}$ as

$$
\begin{aligned}
& \mathscr{D}: \text { minimize } \Phi(\lambda), \text { subject to } \\
& \Psi^{T} \lambda+s=P e_{K} \\
& \lambda \geq 0, \\
& s \geq 0 .
\end{aligned}
$$

where $\lambda$ is the $P \times 1$ dual vector of Lagrange multipliers in the Lagrangian, $e_{K}$ is the $K \times 1$ vector with all entries equal to one, and the scalar $P$ is the number of TAC measurements. Observe that Equation (2.17) is the objective function, Equation (2.18) is the equality constraint function, and the Lagrange multipliers and dual slack variables in Equation (2.19) and Equation (2.20) respectively are the inequality constraint functions.

The Karush-Kuhn-Tucker (KKT) conditions (see Definition A.3.40, Definition A.3.41, and Equation 3.57 in Definition A.3.47) are a system of equations that when solved also solves the primal-dual problem. The KKT conditions 
for this problem are

$$
\begin{gathered}
\Psi^{T} \lambda+s=P e_{K} \\
\Lambda \Psi w^{K}=e_{P} \\
W S e_{K}=0 \\
\left(w^{K}, s\right) \geq 0
\end{gathered}
$$

where $W$ is the $K \times K$ diagonal matrix of weights.

Define $F: \mathbb{R}^{2 K+P} \rightarrow \mathbb{R}^{2 K+P}$ as

$$
\begin{gathered}
F\left(w^{K}, \lambda, s\right)=\left[\begin{array}{c}
\Psi^{T} \lambda+s-P e_{K} \\
\Lambda \Psi w^{K}-e_{P} \\
W S e_{K}
\end{array}\right]=0 \\
\left(w^{K}, s\right) \succeq 0
\end{gathered}
$$

Interior-points refer to the strictly positive pairs, $\left(w^{K}, s\right)>0$, in the KKT conditions in Equation (2.24). Note this is not the same as the joint mixing distribution that is the main topic of this paper. The interior-point methods to solve optimization problems only seeks the pairs $\left(w^{*}, s^{*}\right)$ which are strictly positive feasible solutions to $F\left(w^{K}, \lambda, s\right)$ in Equation (2.25). As Dr. Wright mentions in the text [24], "by respecting these bounds, the methods avoid spurious solutions, which are points that satisfy $F\left(w^{K}, \lambda, s\right)=0$ but not $\left(w^{K}, s\right) \geq 0$." 
The primal-dual interior-point method (see Remark A.3.48) is adjusted depending on the particular optimization problem. The details of the execution of the calculation are involved and not provided here, and the reader should see the references at the beginning of this subsection for a complete explanation. The PDIP method essentially utilizes a path based Newton's method of gradient descent to find feasible solutions to the KKT optimization function $F\left(w^{K}, \lambda, s\right)$ in Equation (2.25).

\subsubsection{NPAG Algorithm Outline}

First, the transdermal alcohol concentration population data $Y_{\epsilon}$ is provided either from simulation, as in the previous section, or from human subjects. Next the grid of support points $\xi^{(n)} \in \Omega^{K}$ is initialized for which each sup-

port point $\xi_{k}^{(n)} \in \Omega$ is contained in a compact rectangular convex hull $\left[a_{1}, b_{1}\right] \times$ $\left[a_{2}, b_{2}\right]$. Recall from Chapter 3 that $K$ is the minimal number of support points necessary to define the maximum likelihood estimator $F^{M L}$. For run-time efficiency it is wise to have minimal grid boundaries chosen. This may be a difficult due to the unknown nature of the distribution. For this research, this was not an issue, because we chose the density functions for $q_{1}$ and $q_{2}$ in advance for the purpose of simulating $Y_{\epsilon}$. We will use this fact to verify the 
convergence of the NPAG algorithm for the selected density functions in the following section. The faure algorithm, which is not provided in this thesis, is used to combine two sets of quasirandom sequences (see YouTube video by Dr. Hayes [7]) into a grid of support points used to initialize the algorithm. Faure sequences are often used in optimization algorithms because they tend to increase the accuracy of initial grids by more thoroughly covering the entire space [25].

Now initialize the bounding constants. We discuss the most significant bounds first. There are two main bounds relating to likelihood that must be understood. The difference between two iterations of log-likelihood calculations on their respective set of probability weights and grid points (see Equation (0.6)) must be less than or equal to the specified upper bound, denoted by $\Delta_{L}=10^{-4}$. In symbols, the log-likelihood converges when

$$
\left|\ell\left(\lambda^{(n+1)}\right)-\ell\left(\lambda^{(n)}\right)\right| \leq \Delta_{L}
$$

Another similar bound, denoted by $\Delta_{F}=10^{-2}$, checks whether the allowable difference between two iterations of log-likelihood calculations is large enough to enter a new loop iteration. In symbols, the particular iteration of the log- 
likelihood is not a candidate for convergence when

$$
\left|\ell\left(\lambda^{(n+1)}\right)-\ell\left(\lambda^{(n)}\right)\right| \geq \Delta_{F}
$$

There is an accuracy variable $e p s$ with an upper bound denoted by $\Delta_{e}=10^{-4}$. This eps variable may be reduced by half during an iteration of the loop when the log-likelihood has converged after a CONDENSE phase is completed. Essentially the $\Delta_{e}$ bound ensures that the grid of support points is reset in the EXPAND algorithm (see Algorithm 8) when the log-likelihood has not converged, but the algorithm has run the necessary number of iterations such that the grid has condensed to an extent such that some support points are too close to other support points. In other words, Equation (2.27) is false, and the inequality

$$
\text { eps } \geq \Delta_{e}
$$

is false. In other words, there has not been enough iterations of the loop when Equation (2.29) is true.

The primary aspects of the code can now be explained. Start the NPAG algorithm by initializing bounding constants. When either Equation (2.28) is true or Equation (2.29) is true, then the loop will be entered. To begin, a $P \times K$ matrix of probabilities is calculated by the $\Psi$ algorithm (see Algorithm 5), as 
in the PDIP section. These values and the grid of support points, $\xi^{(n)}$, are used by the PDIP algorithm to calculate the weights $w^{(n)}$. These results are used to calculate $\ell\left(\lambda^{(n)}\right)$.

Now the CONDENSE algorithm (see Algorithm 7) is called. This algorithm first seeks for support points with weight less that the upper bound of allowable probability, denoted as $\Delta_{w}=10^{-3}$, and removes them from the $\xi^{(n)}$ set. The resulting set of support points is renamed $\xi^{c}$, where the $c$ denotes "condensed" not a set complement.

After this is completed, the counter increases to $n+1, \xi^{c}$ is used by the PDIP algorithm to calculate the weights $w^{(n+1)}$, and subsequently $\ell\left(\lambda^{(n+1)}\right)$ as well. Equation (2.27) can now be checked. If it the inequality is true and the accuracy variable eps is still above $\Delta_{e}$, then eps reduces by half.

Next, if the inequality in Equation (2.29) is false, which means that a sufficient number of iterations have passed, then the inequality in Equation (2.28) must be checked for log-likelihood convergence. If there is convergence, then the loop ends with the current reduced grid of supports $\xi^{c}$ and log-likelihood $\ell\left(\lambda^{(n+1)}\right)$. If there is not convergence, then this means that the grid has condensed, but the algorithm has not found a sufficient log-likelihood/grid of support combination. To address this problem the accuracy variable is re- 
set to allow for the grid to condense further around a new set of grid support points during the next iteration.

Next the EXPAND algorithm (see Algorithm 8) is called. New support points are added, equal to the same number of support points removed. These are within an allowable lower bound of distance between support points, denoted by $\Delta_{D}=10^{-4}$, to the support points of $\xi^{c}$. Recall that the set $\xi^{c}$ contains only support points with sufficient weight. The new set of support points is named $\xi^{e}$, where $e$ denotes "expanded". The loop restarts and $\xi^{e}$ is provided as the new list of supports $\xi^{(n+1)}$ with the $\ell\left(\lambda^{(n+1)}\right)$ log-likelihood. The loop will continue until a stopping condition is met. The stopping conditions are as follows:

Condition 1: At the start of the loop, if the conditions are such that Equation (2.28) is false and Equation (2.29) is false, then the loop terminates and the output is returned.

Condition 2: After the algorithm condenses the grid, if the inequality in Equation (2.29) is false and Equation (2.28) is also false, then the algorithm terminates and the current information output is returned. 
Condition 3: A fail-safe variable called MAXCYCLES is included in the algorithm which acts as an upper bound on the number of allowable iterations. It will cause the main algorithm loop to break out and return the current loglikelihood value. This is useful when the computation time to convergence is untenable. Hence, it is best for this bound to be very large as default to ensure that the actual local maximum likelihood estimation is reported. When MAXCYCLES is set to zero, then the NPAG algorithm runs once and reports the log-likelihood calculation. 


\subsection{Consistency and Convergence of the NPAG}

\section{Algorithm}

It is important to note that the NPAG algorithm will only estimate the maximum log-likelihood for the available data measurements and thus it is possible that the estimate may not be the "true" maximum likelihood estimator $F^{M L}$. Hence, convergence and consistency are important questions to explore regarding the NPAG algorithm.

\subsubsection{Consistency}

The sequence of maximum likelihood estimators $F_{n}^{*}$ of $F^{M L}$ is consistent (see Definition A.1.10) if for every $q \in \Omega$ and for every $\epsilon>0$

$$
\lim _{P \rightarrow \infty} \mathbb{P}\left(\left|F_{P}^{*}(q)-F^{M L}(q)\right| \geq \epsilon\right)=0
$$

where $P$ is the population size. In other words, the probability that the estimates for $F^{M L}$ do not converge to $F^{M L}$, as the number of $P$ data measurements increases, must tend to zero for the maximum likelihood estimator to be consistent. Recall from Equation (0.3) that the $F^{M L}$ estimate is completely dependent upon the number of transdermal alcohol concentration measurements. This is due to the how the space of possible distributions is reduced to 
the space of distributions with at most $K$ points of support, denoted as $\mathscr{F}_{K}$, where $K \leq P$. In Chapter 3, it was discussed how Lindsay [13] accomplished this by way of Carathéodory Theorem (see Theorem A.3.19). The proof of consistency is carried out in a paper written by Kiefer and Wolfowitz titled, "Consistency of the Maximum Likelihood Estimator in the Presence of Infinitely Many Incidental Parameters" [9]. They explain that when the parameters $F^{*}$ meet certain regulatory conditions, then the maximum likelihood estimator is not only consistent, but $F^{*}$ converges to $F^{M L}$ with probability one. The problem presented in this thesis meets the assumptions required in the article, and so it is assumed that this maximum likelihood estimator is consistent.

\subsubsection{Convergence}

The problem of the convergence of feasible estimates for the maximum likelihood, denoted by $F^{*}$, to $F^{M L}$ estimator is very difficult. One of the obstacles arises from the grid of support points. A central aspect of the NPAG algorithm is the choice of an initial grid on a compact space $\left[a_{1}, b_{1}\right] \times\left[a_{2}, b_{2}\right]$. This in combination with the process of the condensing around points with highest probability in this space results in the NPAG algorithm being limited to only estimating a local maximum likelihood. Of course, one can attempt to use a 
very large initial grid, but that may increase the computation time substantially. If possible, it is more efficient to analyse the problem and make an optimal first guess for the initial grid.

The primary method for verifying convergence to this local maximum likelihood was provided in the previously referenced 2019 paper [25]. The research group leveraged directional derivatives of the log-likelihood of $F$ in the direction of the atomic density function centered at each support point. It is denoted as $D_{\delta_{\xi}} \ell(F)$. The idea originates in a text by Fedorov titled "Theory of Optimal Experiments" [4], which covers $D$-optimal design theory. Another connection to Fedorov's $D$-optimal design theory and maximum likelihood estimators is provided by Mallet in a paper titled "A Maximum Likelihood Estimation Method for Random Coefficient Regression Models" [14]. That paper provides an alternative to Lindsay's approach that was discussed in Chapter 3. In fact, according to Schumitzky [21] Lindsay and Mallet worked in concert to develop the theory that reduced the space of distributions to $\mathscr{F}_{K}$.

In order to leverage the directional derivative a function was defined. Let $F$ be any distribution on $\Omega$, the space of parameters for $q$. Then define the 
directional derivative D-Function as

$$
D(\xi, F)=\left(\sum_{i=1}^{P} \frac{p\left(Y_{i} \mid \xi\right)}{p\left(Y_{i} \mid F\right)}\right)-P .
$$

The method by Fedorov requires that $D\left(\xi, F_{k}\right)$ is maximized for every iteration of the NPAG algorithm. Then, it can be shown that $\ell\left(F_{k}\right)$ converges to $\ell\left(F^{M L}\right)$ when regularity conditions are met. In practice, the calculation yields a value which must be very near zero for the convergence of the NPAG algoritm to be verified since this denotes that an extremum is achieved. It should be noted that this is very computationally intensive and there are even longer run-times for higher dimensional arguments. The 2019 article goes on to offer efficiency improvements to the method by Fedorov. They also deviate from Fedorov's method by only completing the calculation for the final iteration of the NPAG algorithm. This makes the run-time more practical since calculating the $D$-function for every iteration is likely results in unduly time costs. According to the researchers, the final iteration can still provide a strong indication that convergence was reached or at least how much the solution deviates from the true value. Lindsay [13] showed that $F^{*}=F^{M L}$ if and only if

$$
\max _{\xi \in \Omega} D\left(\xi, F^{*}\right)=0
$$


Additionally, in the same paper Lindsay showed when

$$
\max _{\xi \in \Omega} D\left(\xi, F^{*}\right) \neq 0
$$

it is still true that

$$
L\left(F^{M L}\right)-L\left(F^{*}\right) \leq \max _{\xi \in \Omega} D\left(\xi, F^{*}\right)
$$

for $F^{*}, F^{M L} \in \mathscr{F}_{K}$. Recall the three chosen joint mixing distributions for $F$ in section one of this chapter. The D-Function plot for each pair is shown in the following pages with the accompanying D-Function output for the maximum. 


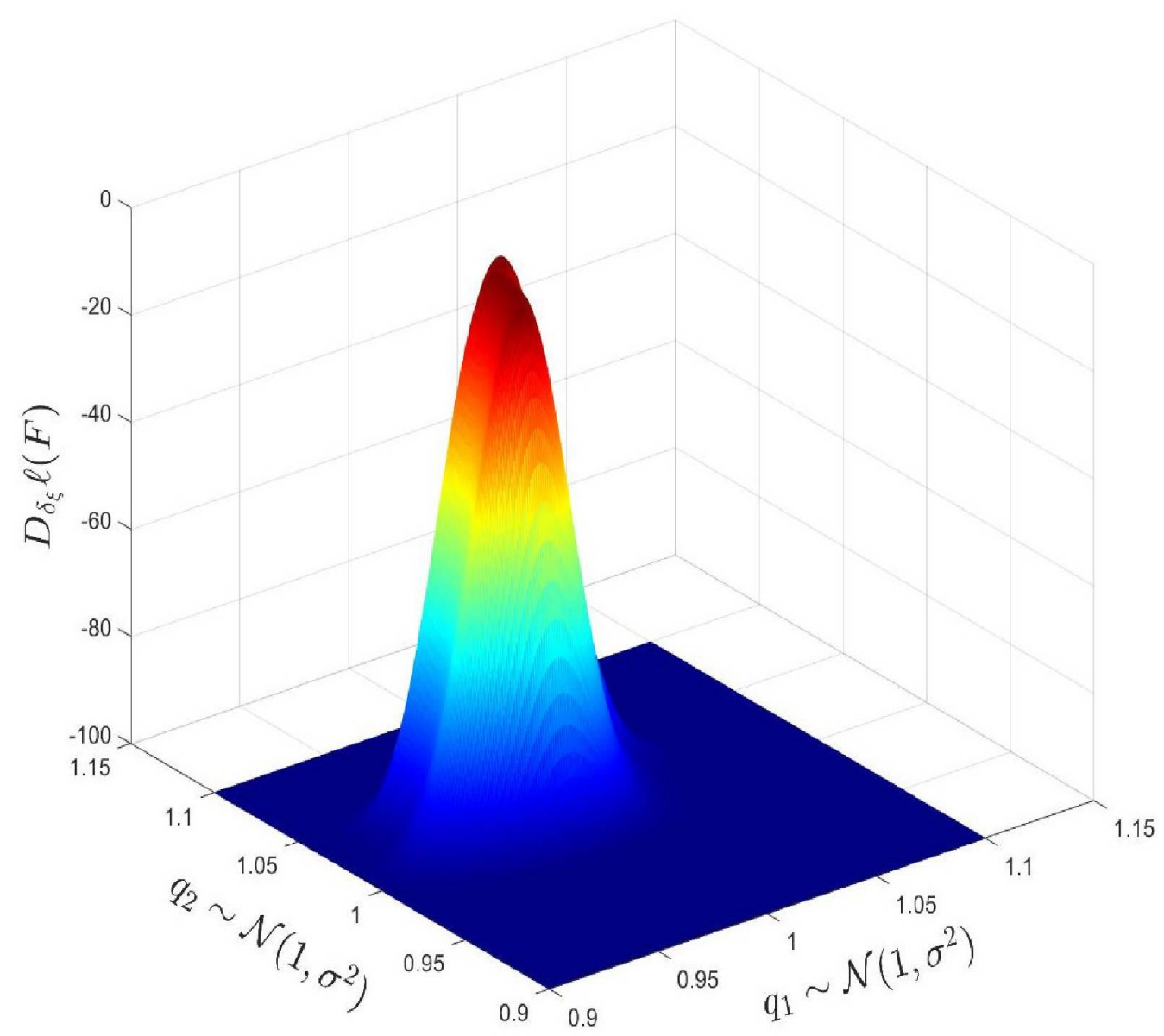

Figure 4.4: Plot of $D$-Function: The Directional Derivative of $\ell(F)$ in the Direction of $\delta_{\xi}$ given $q_{1} \sim \mathscr{N}\left(1, \sigma^{2}\right)$ and $q_{2} \sim \mathscr{N}\left(1, \sigma^{2}\right)$

D-Function $\max =-0.0019$ 


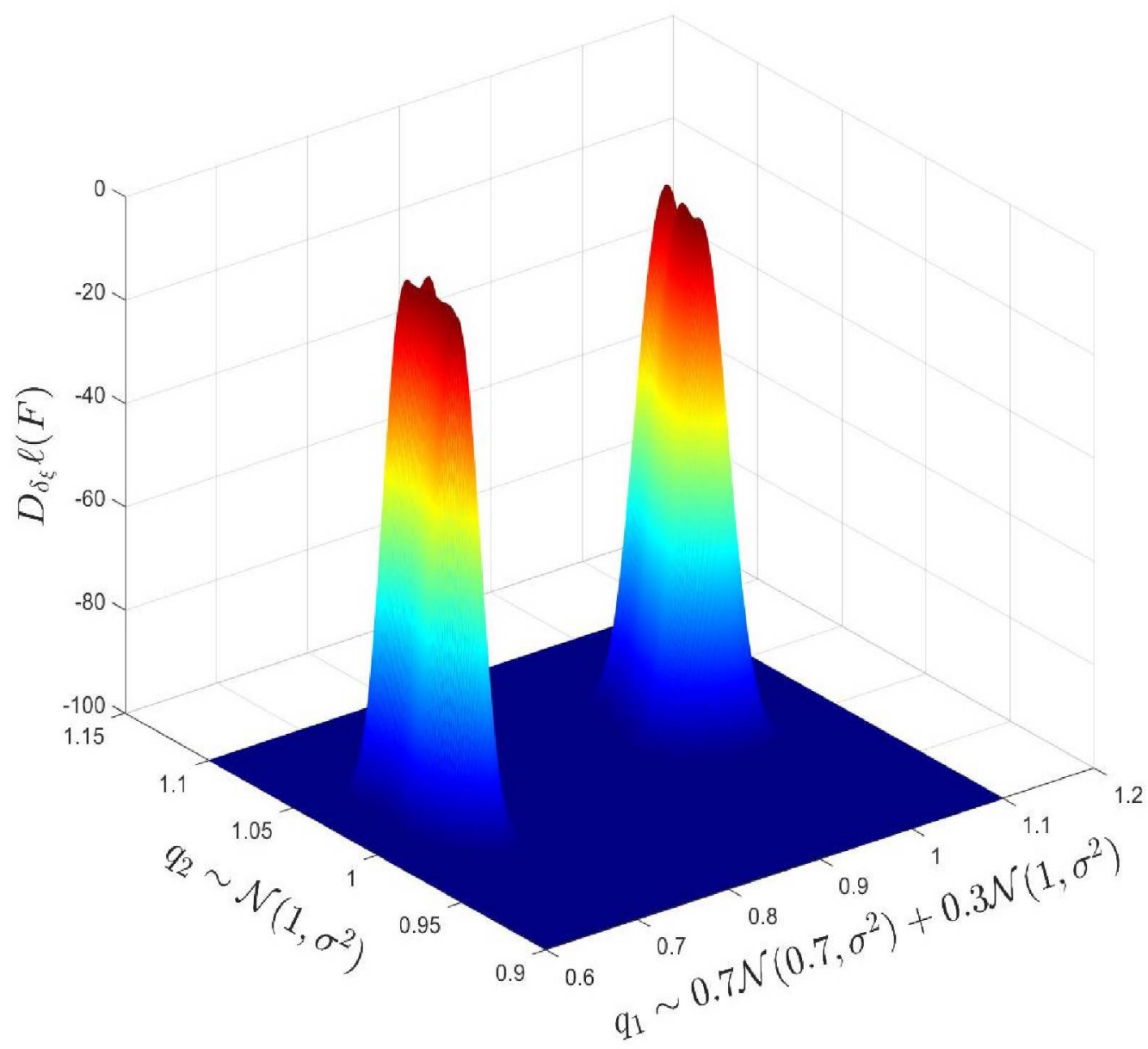

Figure 4.5: Plot of $D$-Function: The Directional Derivative of $\ell(F)$ in the Direction of $\delta_{\xi}$ given $q_{1} \sim 0.7 \mathcal{N}\left(0.7, \sigma^{2}\right)+0.3 \mathcal{N}\left(1, \sigma^{2}\right)$ and $q_{2} \sim \mathscr{N}\left(1, \sigma^{2}\right)$

D-Function $\max =-0.0026$ 


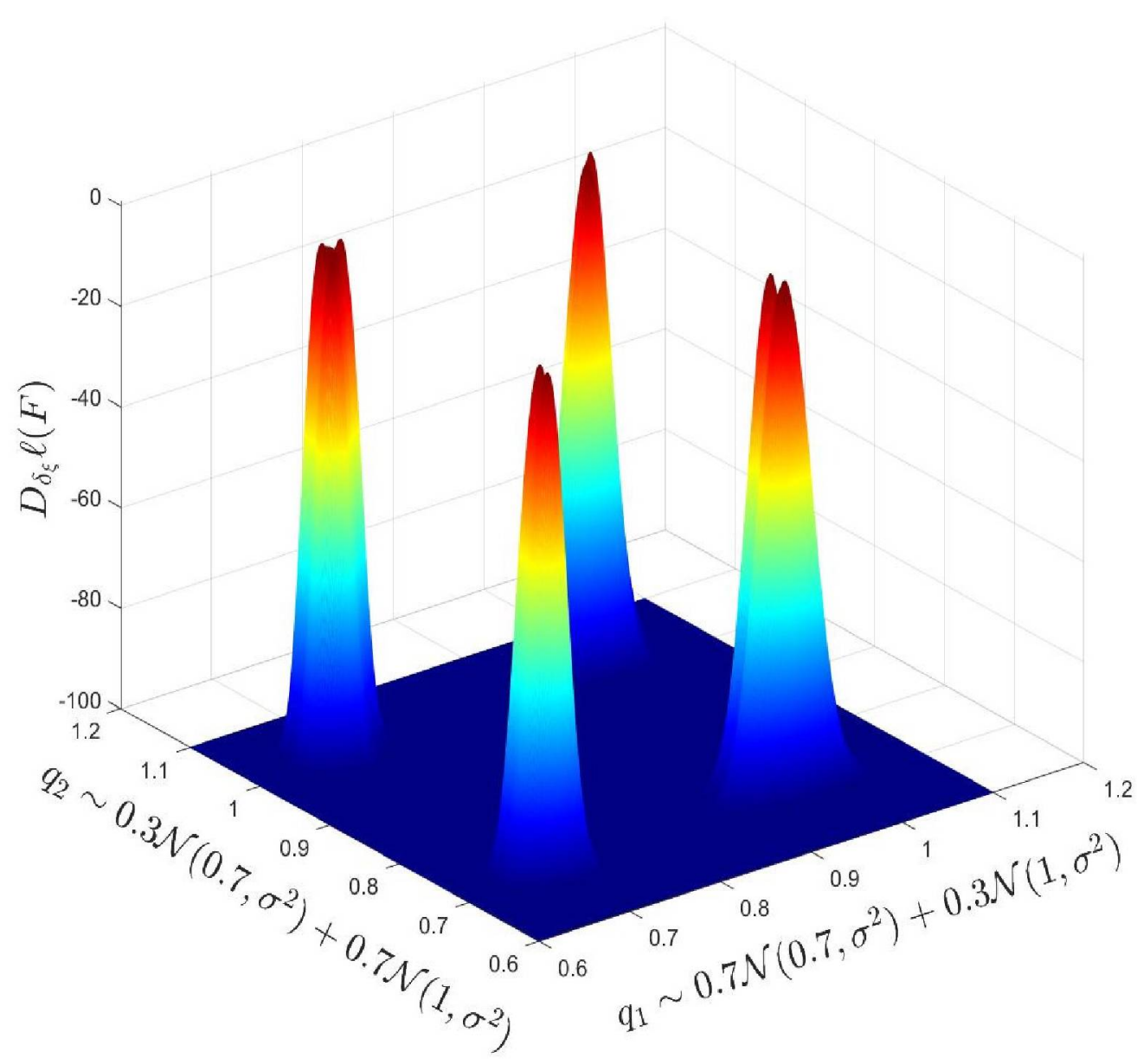

Figure 4.6: Plot of $D$-Function: The Directional Derivative of $\ell(F)$ in the Direction of $\delta_{\xi}$ given $q_{1} \sim 0.7 \mathscr{N}\left(0.7, \sigma^{2}\right)+0.3 \mathscr{N}\left(1, \sigma^{2}\right)$ and $q_{2} \sim 0.3 \mathscr{N}\left(0.7, \sigma^{2}\right)+0.7 \mathscr{N}\left(1, \sigma^{2}\right)$

D-Function $\max =0.0029$ 
As an extra method of verification that the NPAG algorithm is working as expected, the additional plots were created for each of the three chosen joint mixing distribution pairs. Each parameter in each pair has the following plots:

Plot 1: The weighted support points for the maximum likelihood estimate of $F$ compared side-by-side with the density function for the true distribution of $F$ from which the respective parameter was originally sampled.

Plot 2: The density function for the true distribution of $F$ from which the respective parameter was originally sampled overlaid with the weighted support points for the maximum likelihood estimate of $F$.

The figures show very accurate results for the NPAG algorithm estimate for the joint mixing distribution $F$. It is important to observe that the actual estimate for $q_{1}$ or $q_{2}$ is the linear combination of all of these weights at their respective support point. One visual obstacle occurs in Plot 2 when comparing the maximum likelihood estimate of $F$ with the true distribution of $F$. The weights are proportional to probabilities and as such they fall far below the peak of 
the true distribution of $F$. This is somewhat remedied in the respective Plot 1 where each plot is emphasized on an appropriately scaled axis. 


\section{Pair 1: Plot 1 for $\mathrm{q}_{1}$}

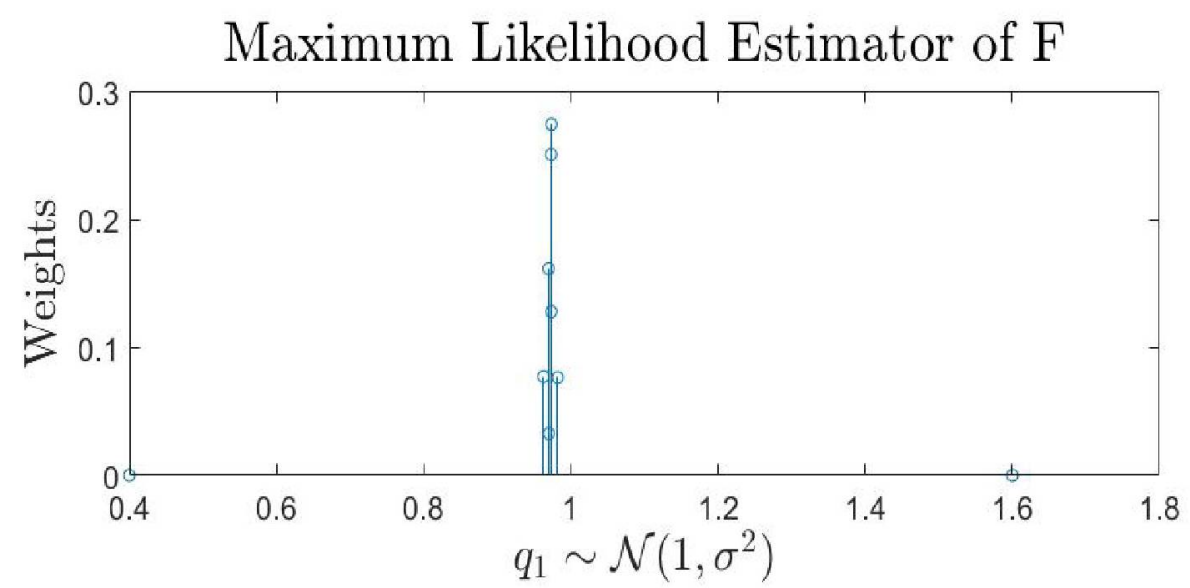

Density Function of the True Distribution F

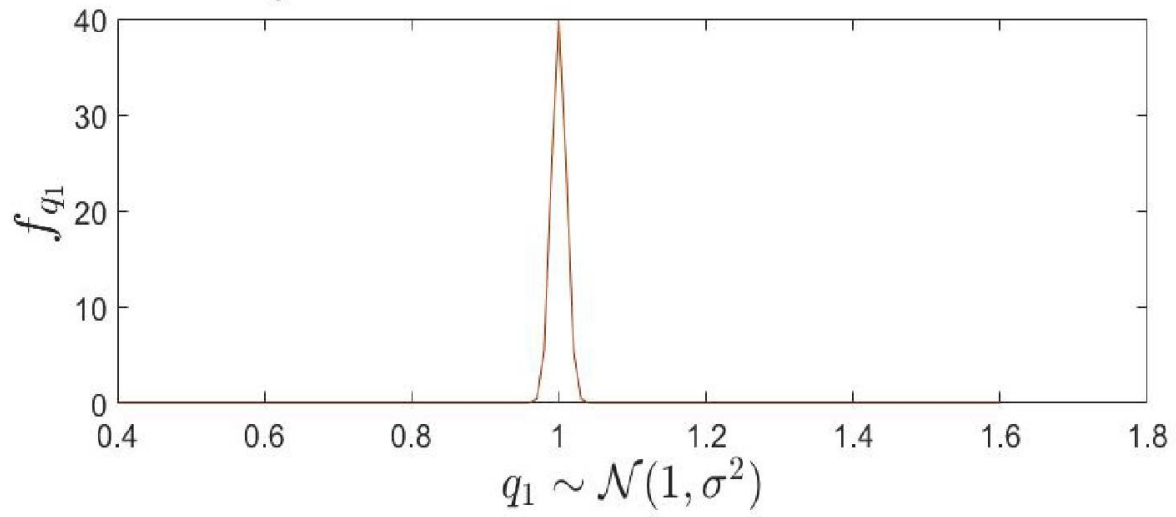

Figure 4.7: Comparison plot of the Maximum Likelihood Estimator of $F$ and the Density Function $f_{q_{1}}$ of the True Distribution of $F$ given $q_{1} \sim \mathscr{N}\left(1, \sigma^{2}\right)$ and $q_{2} \sim \mathscr{N}\left(1, \sigma^{2}\right)$

Weighted Estimate: $q_{1}=0.9724$ 


\section{Pair 1: Plot 2 for $q_{1}$}

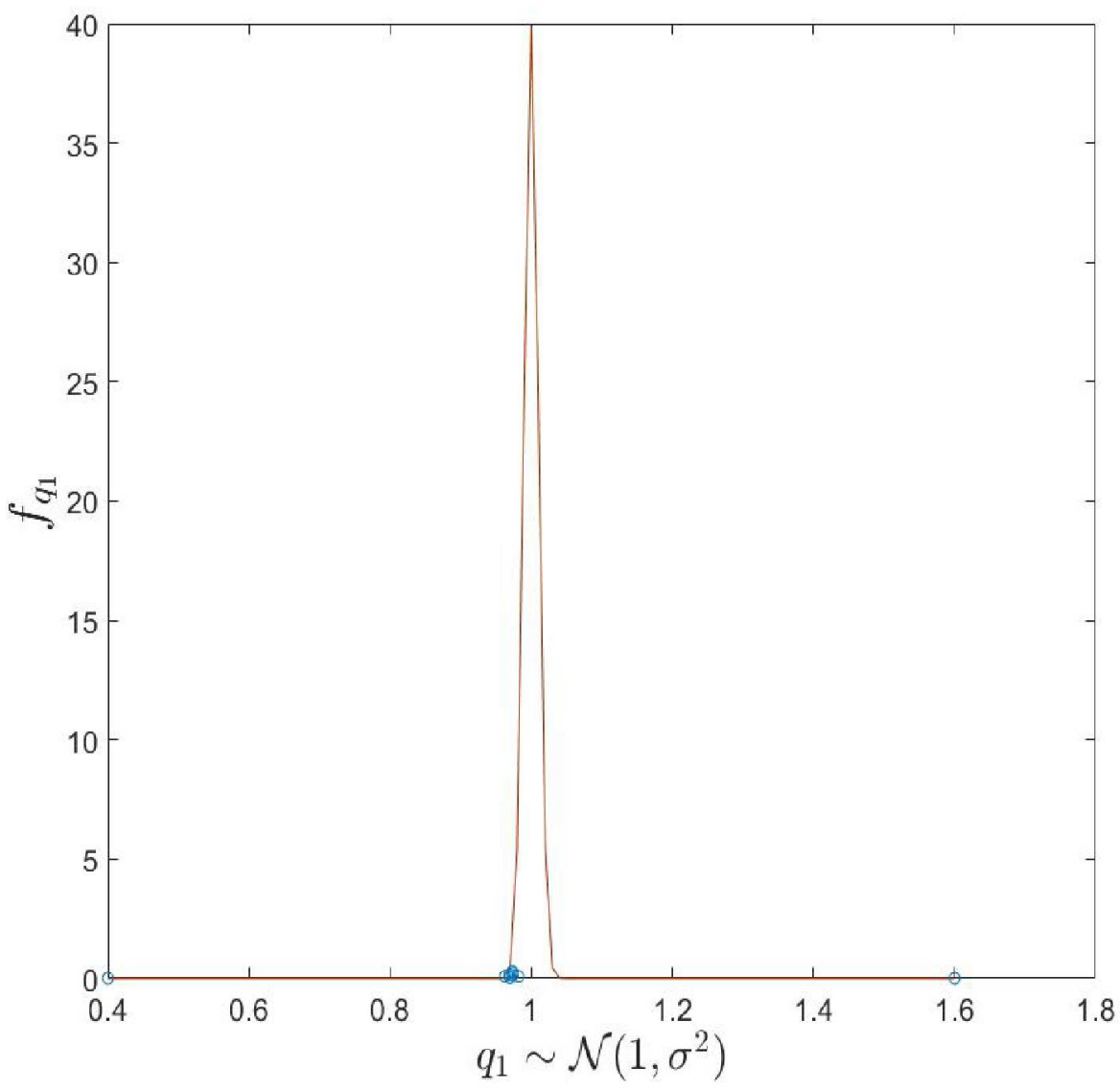

Figure 4.8: Plot of the Density Function $f_{q_{1}}$ of the True Distribution of $F$ Overlaid with the Maximum Likelihood Estimator of $F$ given $q_{1} \sim \mathscr{N}\left(1, \sigma^{2}\right)$ and $q_{2} \sim \mathscr{N}\left(1, \sigma^{2}\right)$

Weighted Estimate: $q_{1}=0.9724$ 


\section{Pair 1: Plot 1 for $\mathrm{q}_{2}$}

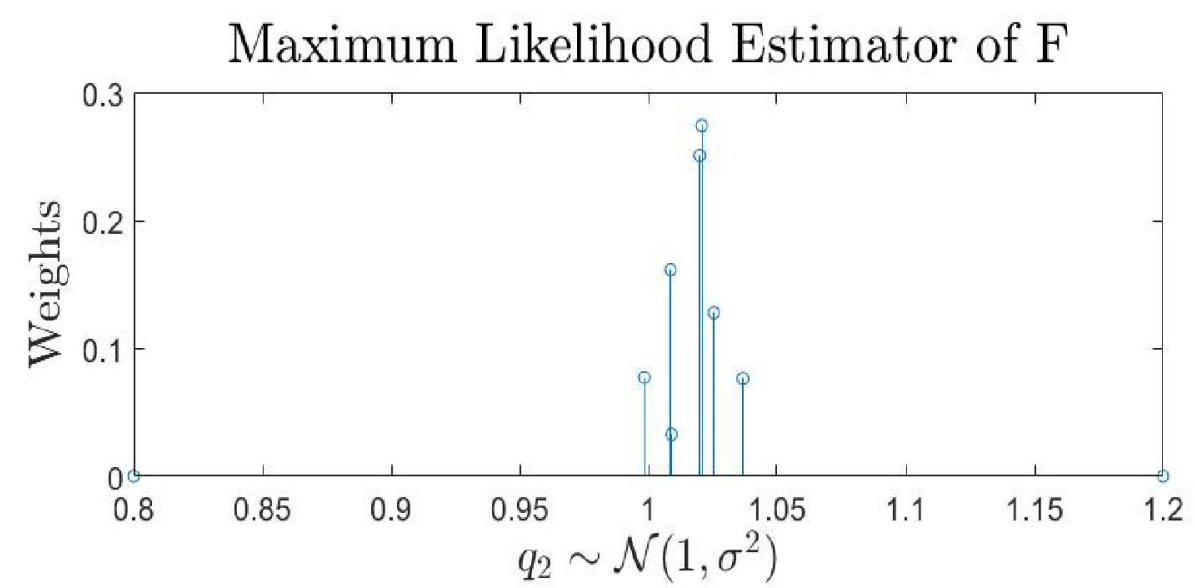

Density Function of the True Distribution F

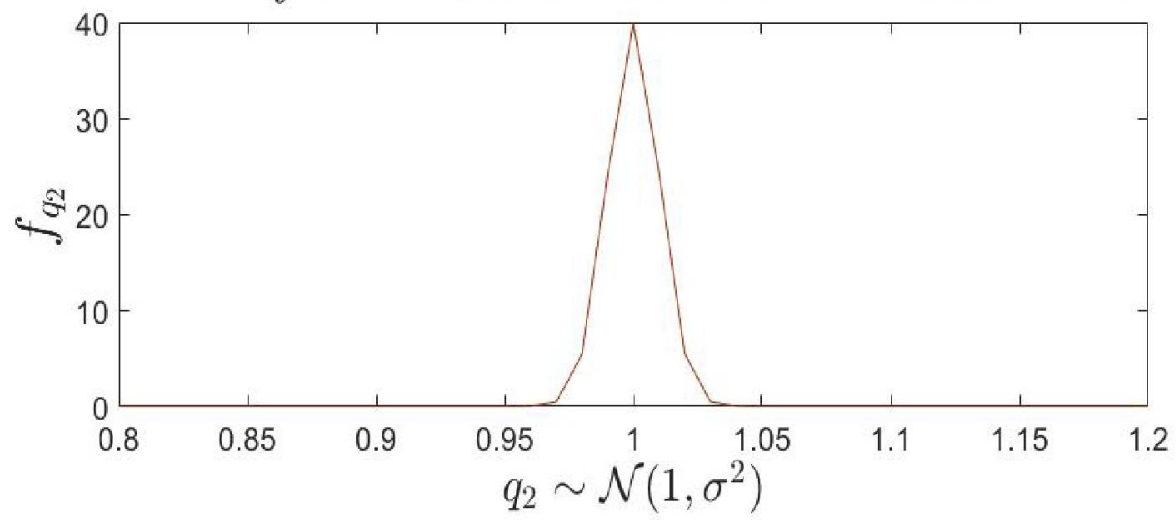

Figure 4.9: Comparison plot of the Maximum Likelihood Estimator of $F$ and the Density Function $f_{q_{2}}$ of the True Distribution of $F$ given $q_{1} \sim \mathscr{N}\left(1, \sigma^{2}\right)$ and $q_{2} \sim \mathscr{N}\left(1, \sigma^{2}\right)$

Weighted Estimate: $q_{2}=1.0183$ 


\section{Pair 1: Plot 2 for $\mathrm{q}_{2}$}

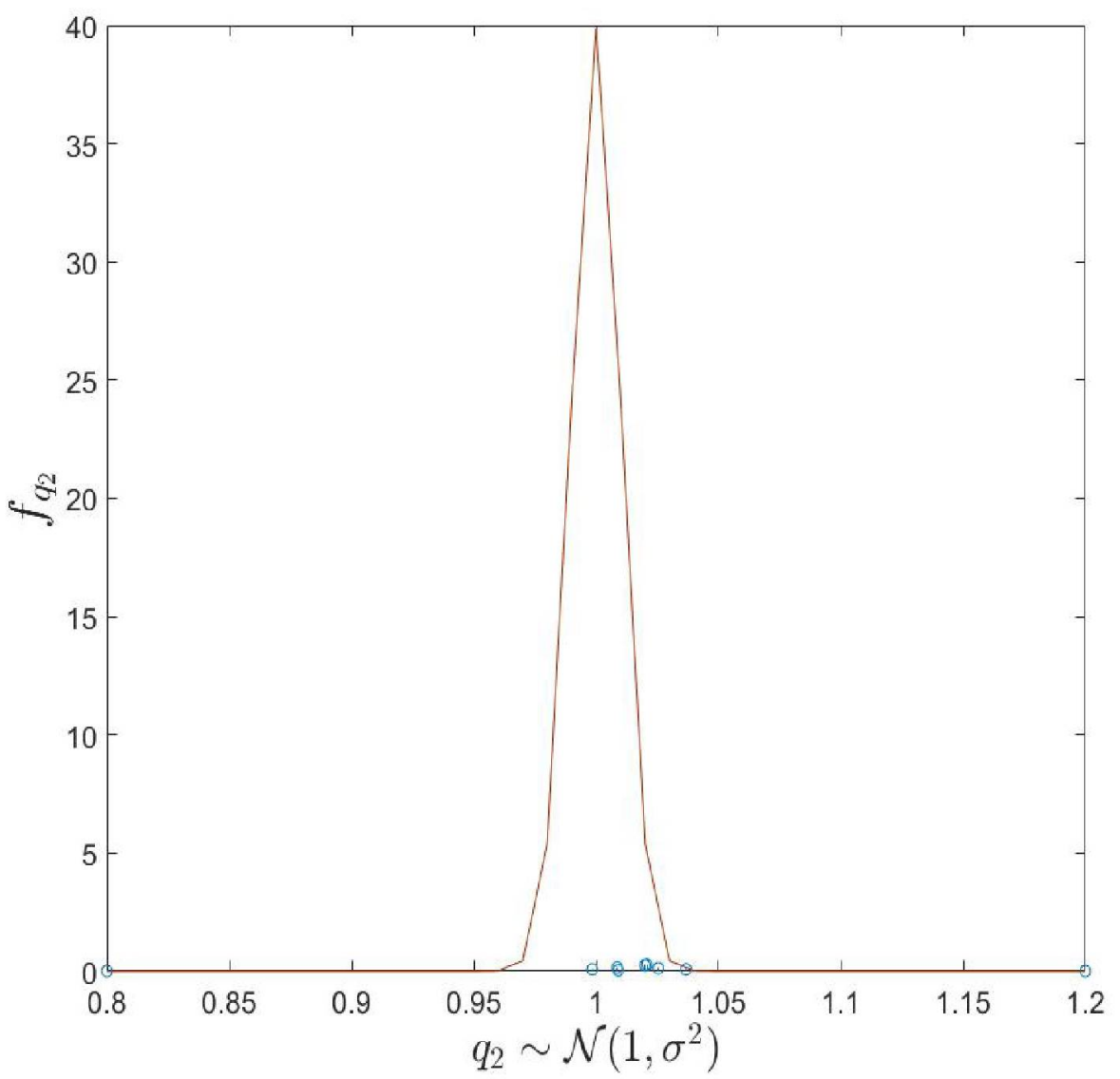

Figure 4.10: Plot of the Density Function $f_{q_{2}}$ of the True Distribution of $F$ Overlaid with the Maximum Likelihood Estimator of $F$ given $q_{1} \sim \mathscr{N}\left(1, \sigma^{2}\right)$ and $q_{2} \sim \mathscr{N}\left(1, \sigma^{2}\right)$

Weighted Estimate: $q_{2}=1.0183$ 


\section{Pair 2: Plot 1 for $\mathrm{q}_{1}$}

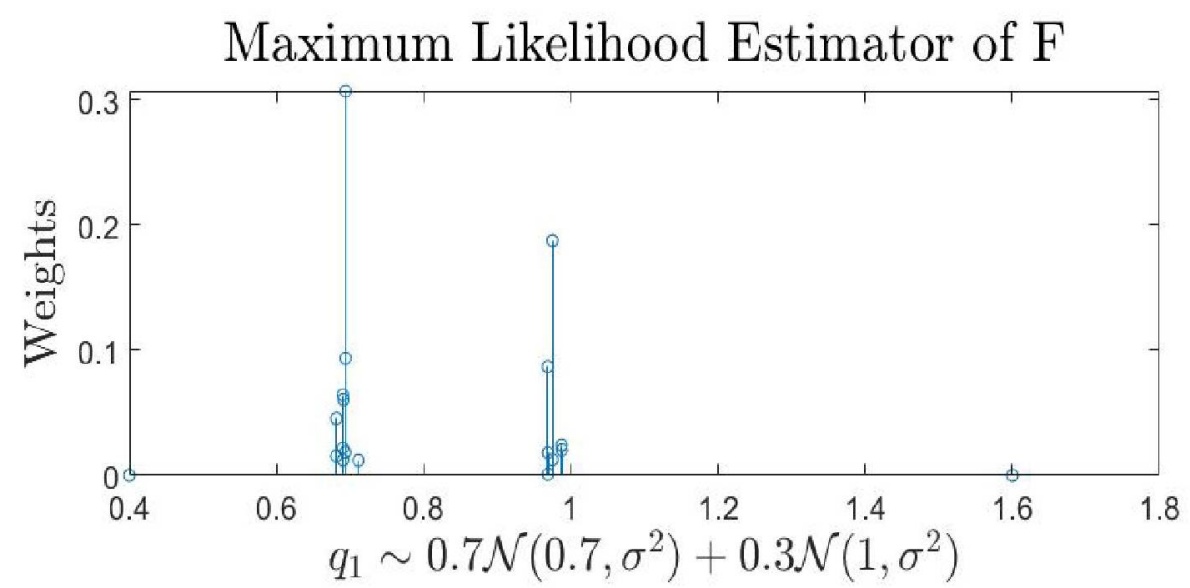

Density Function of the True Distribution F

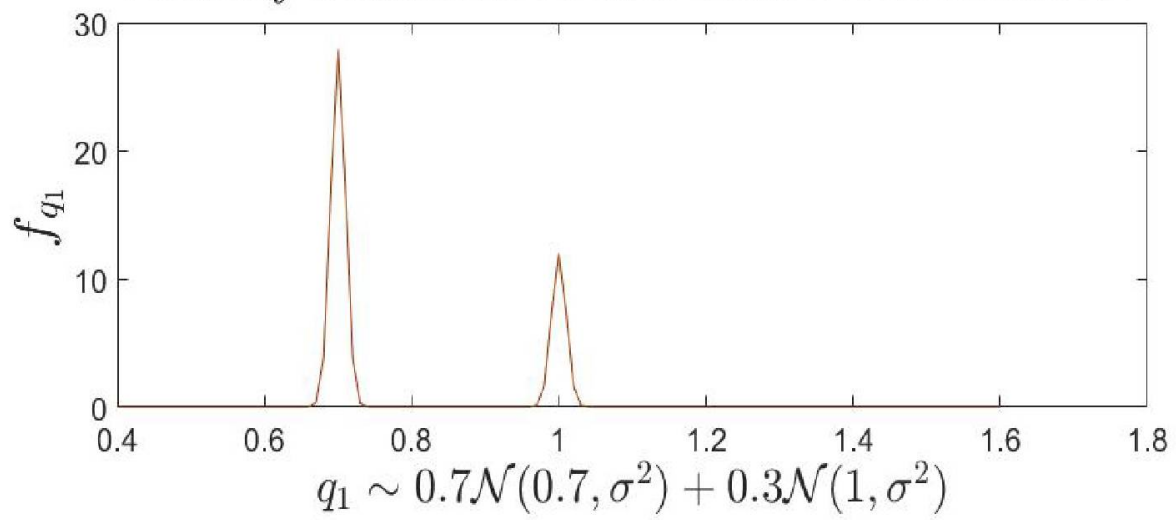

Figure 4.11: Comparison plot of the Maximum Likelihood Estimator of $F$ and the Density Function $f_{q_{1}}$ of the True Distribution of $F$ given $q_{1} \sim 0.7 \mathscr{N}\left(0.7, \sigma^{2}\right)+0.3 \mathscr{N}\left(1, \sigma^{2}\right)$ and $q_{2} \sim \mathcal{N}\left(1, \sigma^{2}\right)$

Weighted Estimate: $q_{1}=0.7909$ 
Pair 2: Plot 2 for $\mathrm{q}_{1}$

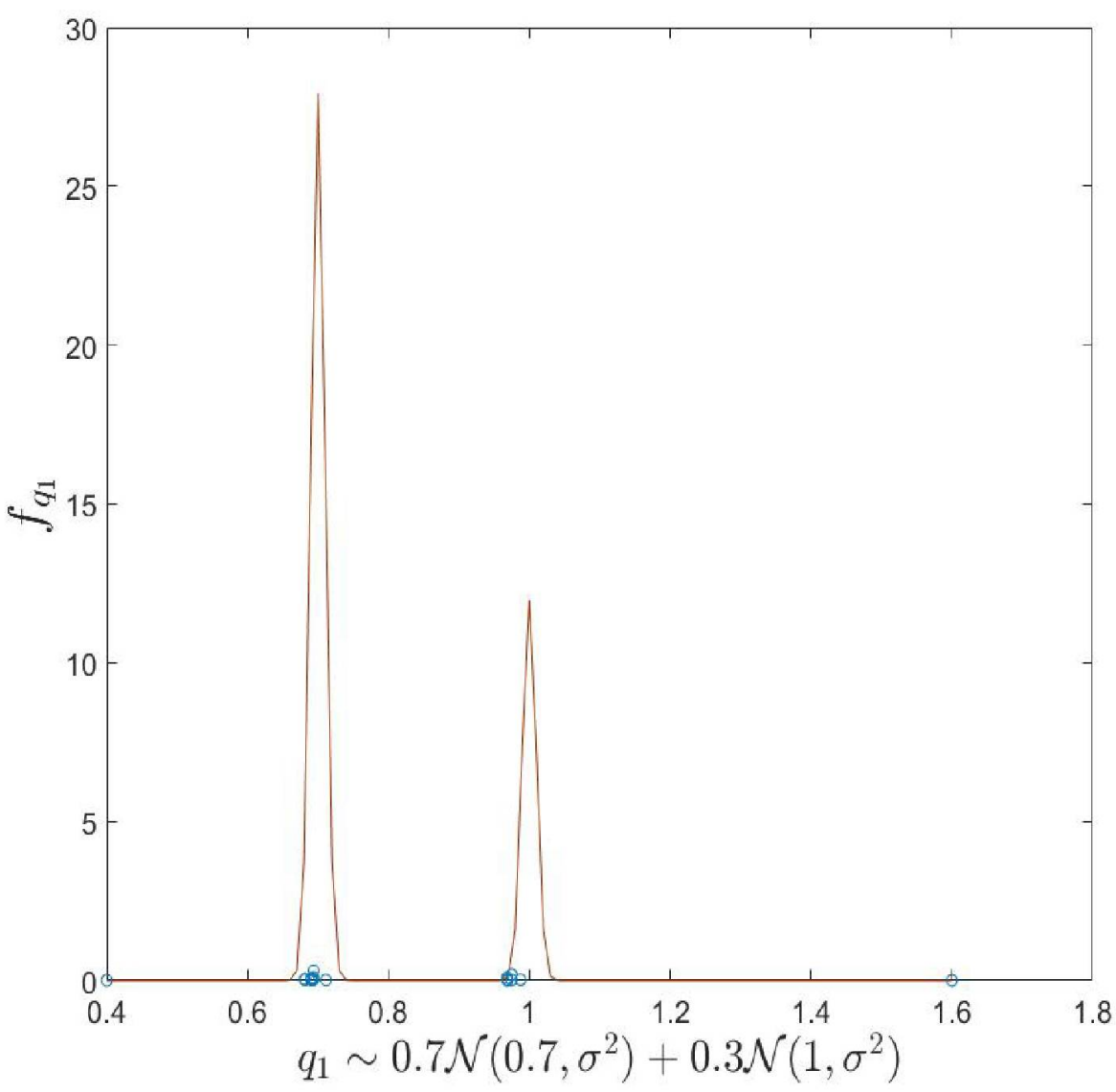

Figure 4.12: Plot of the Density Function $f_{q_{1}}$ of the True Distribution of $F$ Overlaid with the Maximum Likelihood Estimator of $F$ given $q_{1} \sim 0.7 \mathscr{N}\left(0.7, \sigma^{2}\right)+0.3 \mathscr{N}\left(1, \sigma^{2}\right)$ and $q_{2} \sim \mathscr{N}\left(1, \sigma^{2}\right)$

Weighted Estimate: $q_{1}=0.7909$ 


\section{Pair 2: Plot 1 for $\mathrm{q}_{2}$}

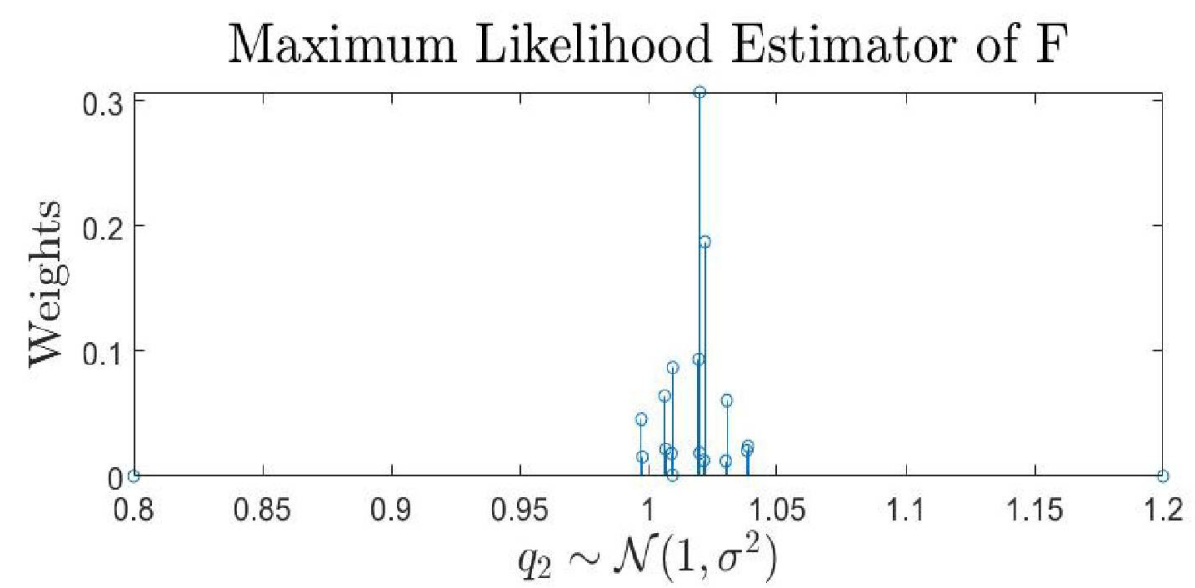

Density Function of the True Distribution F

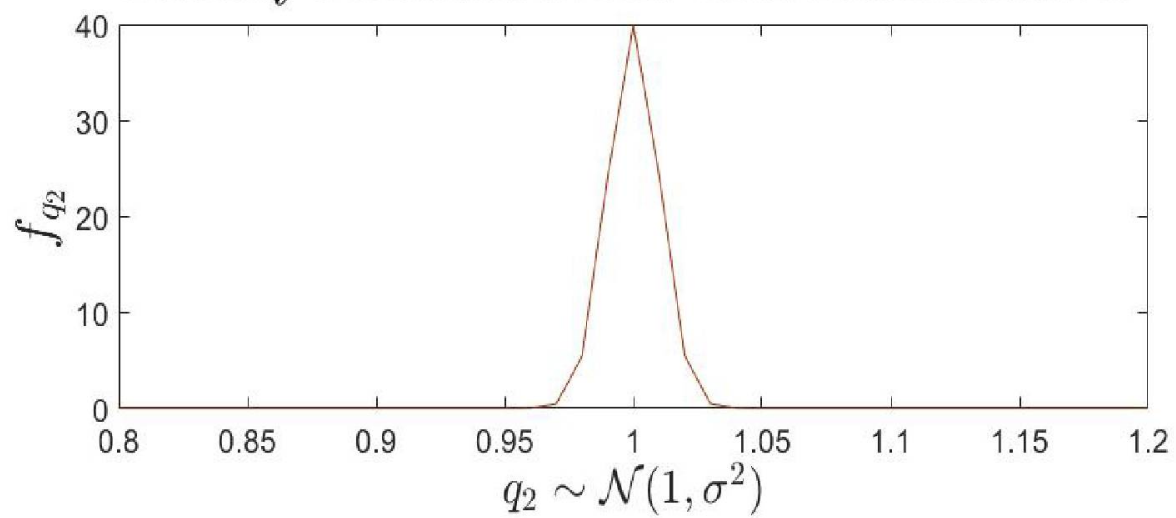

Figure 4.13: Comparison plot of the Maximum Likelihood Estimator of $F$ and the Density Function $f_{q_{2}}$ of the True Distribution of $F$ given $q_{1} \sim 0.7 \mathscr{N}\left(0.7, \sigma^{2}\right)+0.3 \mathscr{N}\left(1, \sigma^{2}\right)$ and $q_{2} \sim \mathscr{N}\left(1, \sigma^{2}\right)$

Weighted Estimate: $q_{2}=1.0183$ 


\section{Pair 2: Plot 2 for $q_{2}$}

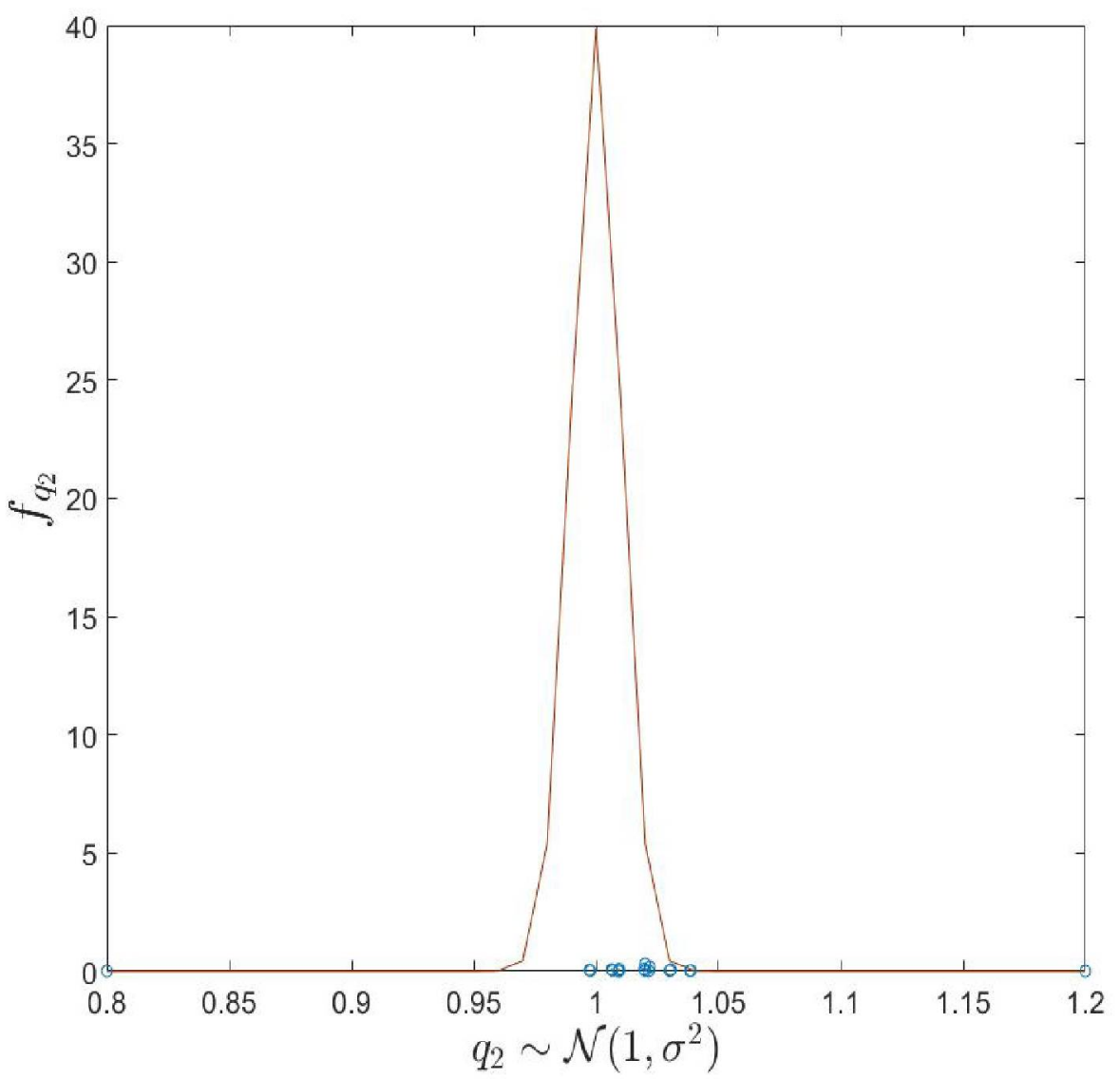

Figure 4.14: Plot of the Density Function $f_{q_{2}}$ of the True Distribution of $F$ Overlaid with the Maximum Likelihood Estimator of $F$ given $q_{1} \sim 0.7 \mathscr{N}\left(0.7, \sigma^{2}\right)+0.3 \mathscr{N}\left(1, \sigma^{2}\right)$ and $q_{2} \sim \mathscr{N}\left(1, \sigma^{2}\right)$

Weighted Estimate: $q_{2}=1.0183$ 
Pair 3: Plot 1 for $\mathrm{q}_{1}$

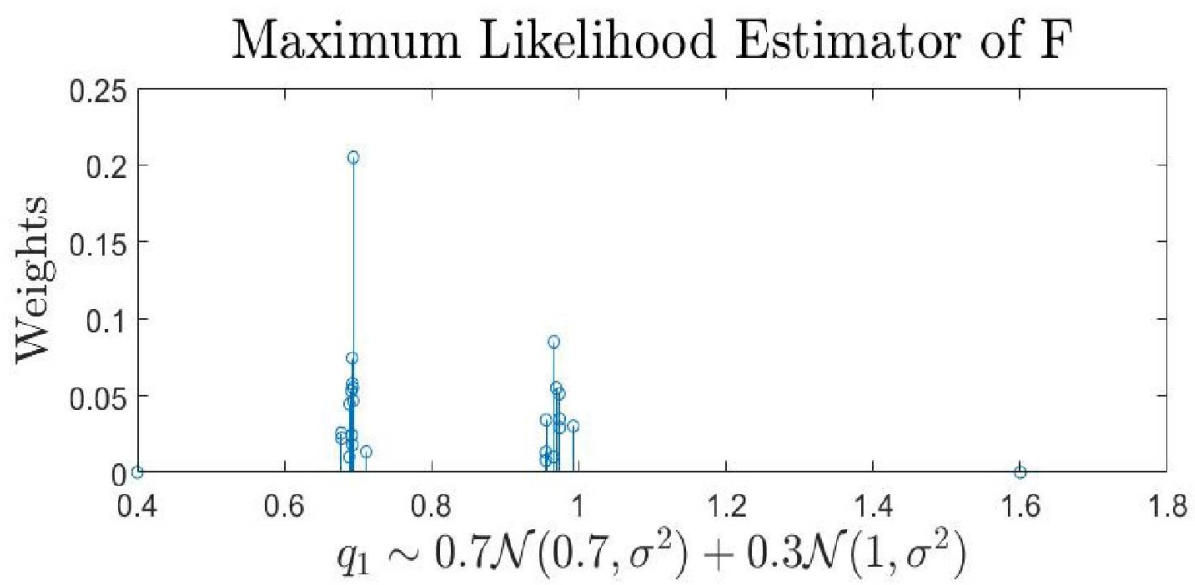

Density Function of the True Distribution F

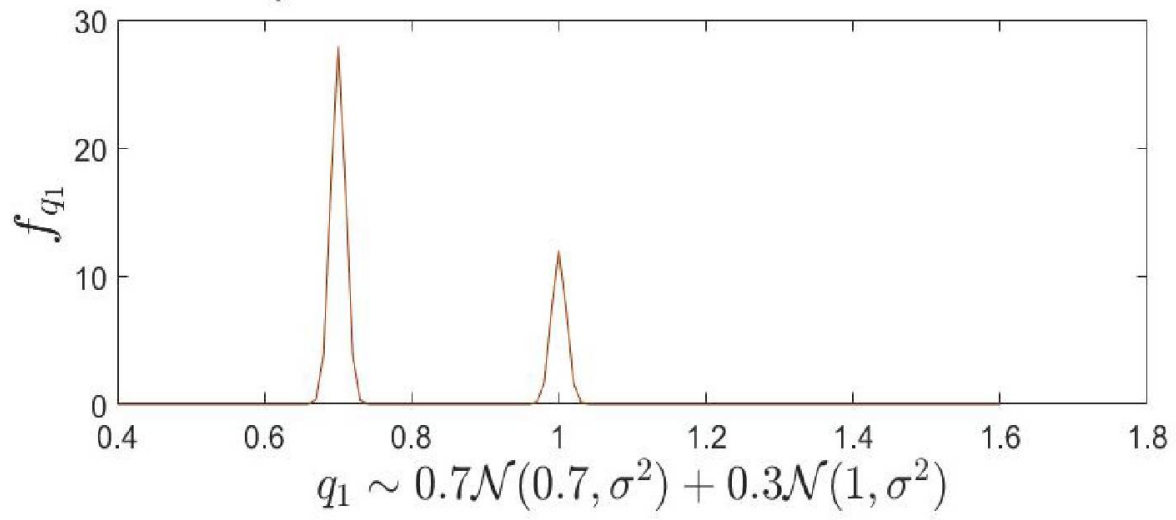

Figure 4.15: Comparison plot of the Maximum Likelihood Estimator of $F$ and the Density Function $f_{q_{1}}$ of the True Distribution of $F$ given $q_{1} \sim 0.7 \mathscr{N}\left(0.7, \sigma^{2}\right)+0.3 \cdot \mathcal{N}\left(1, \sigma^{2}\right)$ and $q_{2} \sim 0.3 \mathscr{N}\left(0.7, \sigma^{2}\right)+0.7 \mathscr{N}\left(1, \sigma^{2}\right)$

Weighted Estimate: $q_{1}=0.7888$ 
Pair 3: Plot 2 for $\mathrm{q}_{1}$

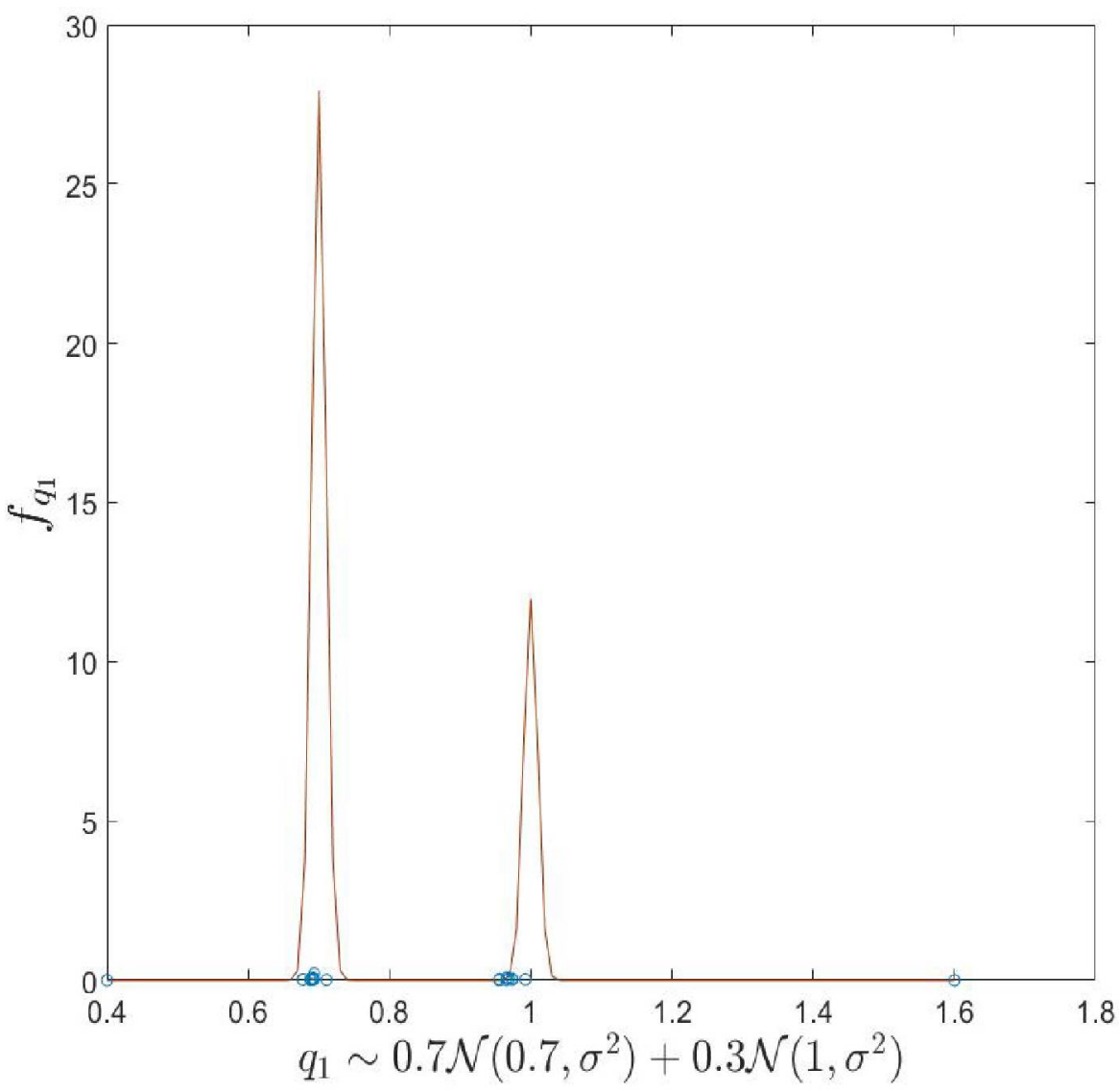

Figure 4.16: Plot of the Density Function $f_{q_{1}}$ of the True Distribution of $F$ Overlaid with the Maximum Likelihood Estimator of $F$ given $q_{1} \sim 0.7 \mathscr{N}\left(0.7, \sigma^{2}\right)+0.3 \mathscr{N}\left(1, \sigma^{2}\right)$ and $q_{2} \sim 0.3 \mathscr{N}\left(0.7, \sigma^{2}\right)+0.7 \mathscr{N}\left(1, \sigma^{2}\right)$

Weighted Estimate: $q_{1}=0.7888$ 


\section{Pair 3: Plot 1 for $\mathrm{q}_{2}$}

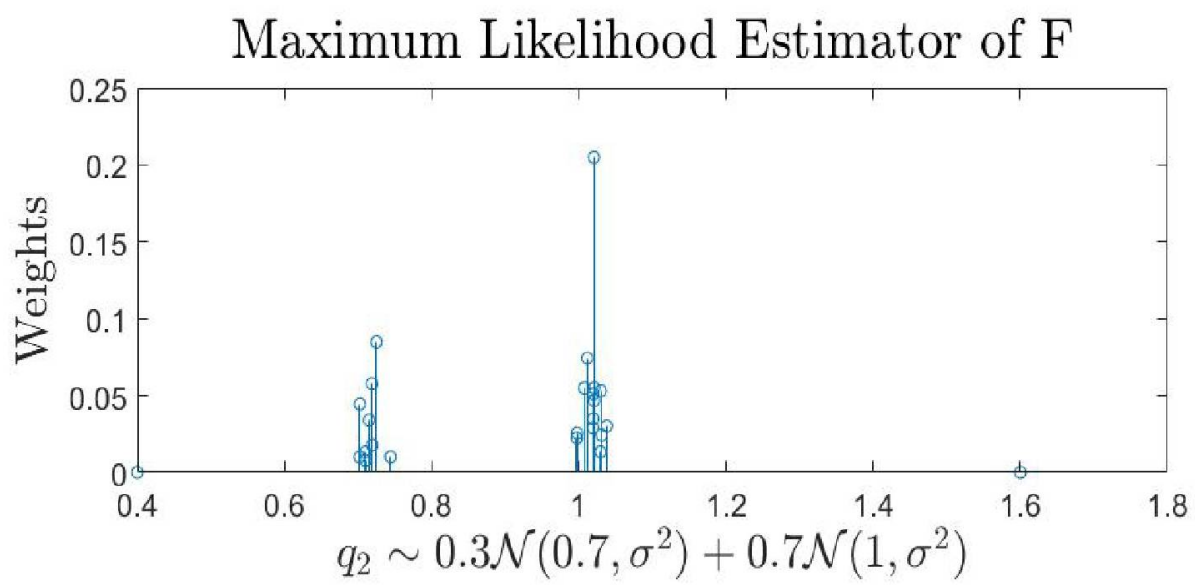

Density Function of the True Distribution F

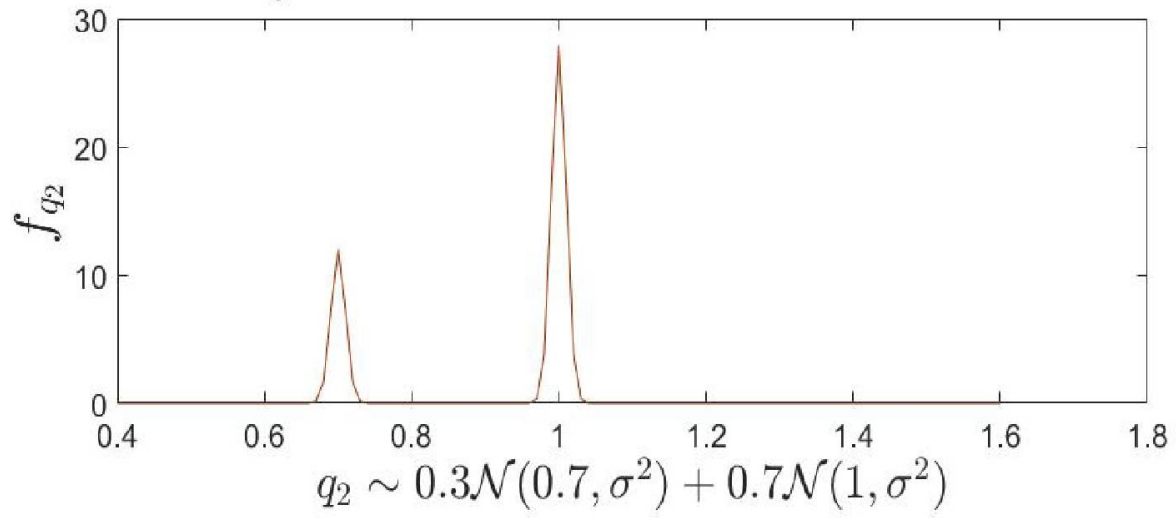

Figure 4.17: Comparison plot of the Maximum Likelihood Estimator of $F$ and the Density Function $f_{q_{2}}$ of the True Distribution of $F$ given $q_{1} \sim 0.7 \mathscr{N}\left(0.7, \sigma^{2}\right)+0.3 \mathscr{N}\left(1, \sigma^{2}\right)$ and $q_{2} \sim 0.3 \mathcal{N}\left(0.7, \sigma^{2}\right)+0.7 \mathcal{N}\left(1, \sigma^{2}\right)$

Weighted Estimate: $q_{2}=0.9343$ 
Pair 3: Plot 2 for $\mathrm{q}_{2}$

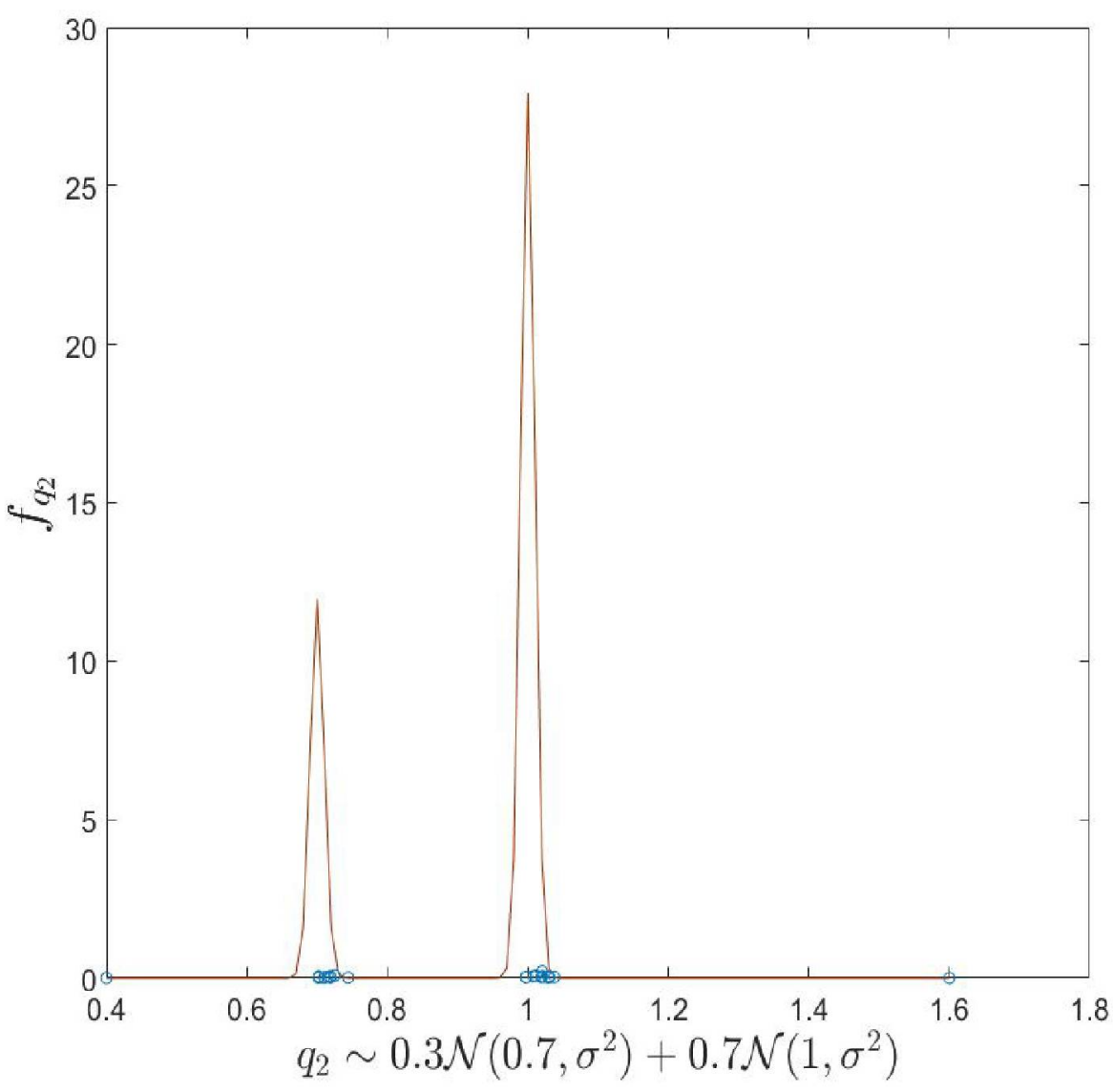

Figure 4.18: Plot of the Density Function $f_{q_{2}}$ of the True Distribution of $F$ Overlaid with the Maximum Likelihood Estimator of $F$ given $q_{1} \sim 0.7 \mathscr{N}\left(0.7, \sigma^{2}\right)+0.3 \mathscr{N}\left(1, \sigma^{2}\right)$ and $q_{2} \sim 0.3 \mathscr{N}\left(0.7, \sigma^{2}\right)+0.7 \mathscr{N}\left(1, \sigma^{2}\right)$

Weighted Estimate: $q_{2}=0.9343$ 


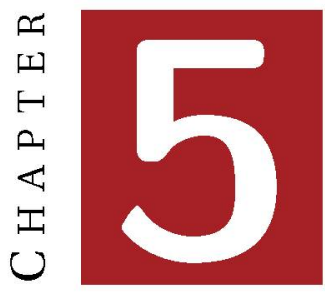

\section{Conclusions}

This paper developed a nonparametric estimation algorithm that calculated the joint mixing distribution of the parameters of a heat equation model via a maximum likelihood method which accounted for measurement error. The results show that there is a relation between blood alcohol concentration and transdermal alcohol concentration.

A relevant summary of the research history of transdermal alcohol concentration was provided in Chapter 1. The most prevalent alcohol biosensors were listed. Furthermore, we highlighted the benefits and challenges that face the technology. The role of the NIAAA government institute in the development of new technology was discussed showing how close we are to hav- 
ing a sufficiently advanced option introduced into the market within the next decade.

In Chapter 2 a heat equation diffusion model was developed to simulate the transdermal alcohol concentration observations needed in subsequent chapters. This model accounted for normal measurement error due to things like sweat and oil at the epidermis. In this model, the drinking schedule and parameters of diffusivity for an individual were identified as the main factors that determine the simulated values. Matlab programs were written to calculate these algorithms which can be read in Appendix B under Algorithm 1 through 3 .

The theory established in Chapter 3 allowed the problem of finding a maximum likelihood estimate of the joint mixing distribution for the parameters of diffusivity to be reduced to the space of distributions with at most $K$ points of support; with $K \leq P$ where $P$ is the population size.

We carried out the simulation in Chapter 4 from data set up in Chapter 2 and verified convergence for three joint mixing distributions for $F$ that fit various biological scenarios. These TAC simulations were then analyzed with an assumption that all 100 instances had identical drinking schedules. Next a convex optimization problem was formulated to calculate the maximum like- 
lihood in a local convex hull of support points. This was accomplished using the nonparametric adapted grid (NPAG) algorithm implemented in Algorithms 4 through 8 . This effective algorithm used a primal-dual interior-point method to calculate the probability of a specific point of support (see Algorithm 5). Ultimately, the joint mixing distribution for the parameters of diffusivity is estimated and graphs are provided that display the success of the calculation for each of the three aforementioned distributions. The questions of the consistency of the maximum likelihood estimator and convergence of feasible distributions to $F^{M L}$ are addressed.

We compiled the relevant definitions in Appendix A. This is of great value since the research spans several fields of study. Each algorithm is explained in detail with any relevant variables or constants in Appendix B. We expended a significant amount of time learning the necessary probability, statistics, functional analysis, convex analysis, numerical analysis, and partial differential equation theory. 


\subsection{Future Work}

There remains many possible expansions to this research. Certainly alternative heat equation discretization methods can and should be investigated for possible reductions in algorithm complexity or increases in accuracy. Use of the finite difference method was considered, but the finite element method was chosen since much of the mathematics was already established in Dr. Melike Sirlanci's dissertation. Plans to return to this mathematically rich method are ongoing for further research if only to corroborate the findings of the finite element method.

The error added to the simulated alcohol measurements in Algorithm 1 was chosen to be normally distributed to fit simple biological variation. There is a plan to substitute this for a stochastic error model, probably using Brownian motion or some variation on that. It is thought that this may better introduce natural randomness seen in complex biological and or environmental systems.

A natural subsequent step will be to consider human subject data in the place of simulated data. Not only will this be a strong test of the NPAG algorithm, but it will also allow for discovering some insight into the real joint mixing distribution for a large population. Once the human subject data for 
a large enough population is provided to the NPAG algorithm, it will provide a good estimate for the larger human populace. Essentially this is a type of machine learning where this human subject data trains the system and more random data can be used to verify the model's accuracy and update the estimate for the parameters of diffusivity. It is an important observation that population data could also take the form of multiple drinking episodes from a single individual.

A Bayesian approach can then be employed as well. Suppose we have a new subject and we would like to calibrate the device to their personal diffusion constants. The maximum likelihood estimate acts as a prior distribution while the new data taken for this subject can be used to calculate their likelihood. This likelihood can be multiplied by the prior distribution to ascertain a posterior distribution for the individual. The mean of this new posterior distribution could act as the new calibrated diffusion constants. See Figure 5.1. 


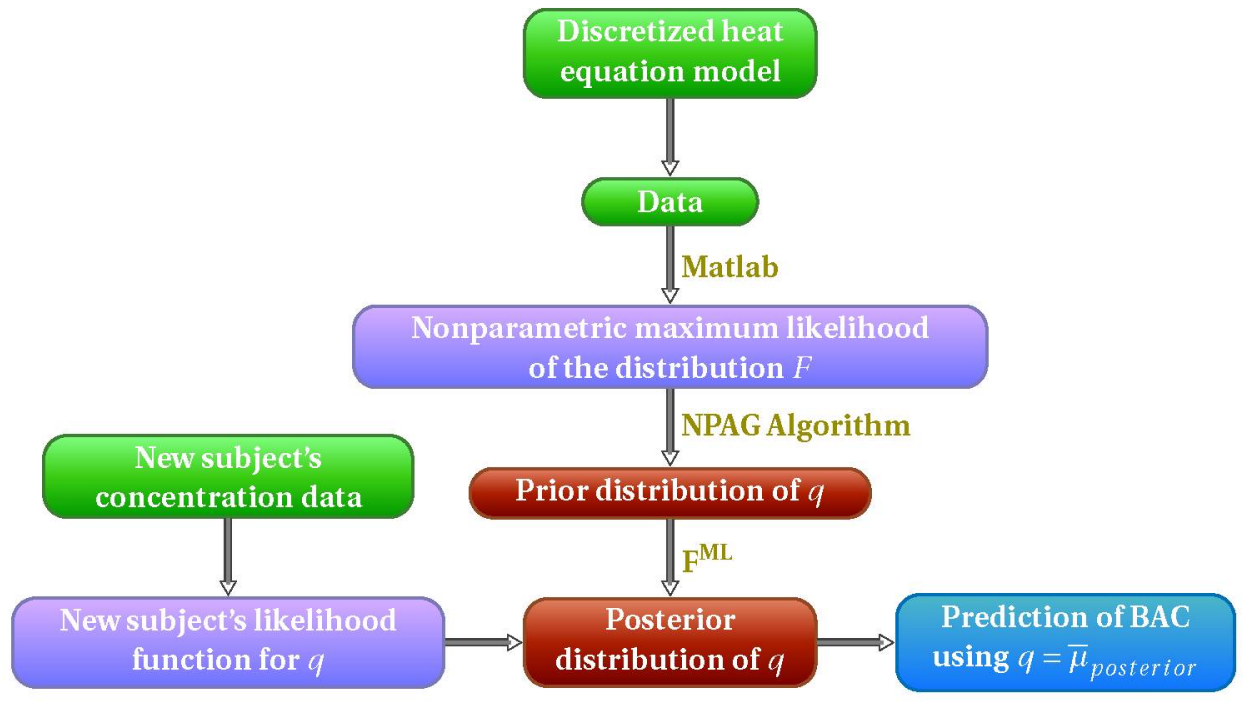

Figure 5.1: Bayesian Calibration for an Individual 


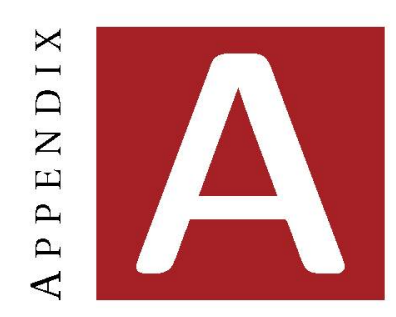

\section{BACKGROUND DEFINITIONS}

This chapter provides a relevant framework of selected definitions, from a variety of well-referenced sources, that gives the necessary applied and theoretical mathematical and statistical background necessary for this thesis.

\section{A.1 Probability and Statistics}

Definition A.1.1 (Joint Probability Distribution) (pg. 177 [1])

The random vector $\mathbf{X}=\left(X_{1}, X_{2}, \ldots, X_{n}\right)$ has a sample space that is a subset of $\mathbb{R}^{n}$

If $\mathbf{X}$ is a discrete random vector, then the joint probability mass function 
(pmf) of $\mathbf{X}$ is the function defined by

$$
f(\boldsymbol{x})=f\left(x_{1}, x_{2}, \ldots, x_{n}\right)=\mathbb{P}\left(X_{1}=x_{1}, X_{2}=x_{2}, \ldots, X_{n}=x_{n}\right)
$$

for any $\mathbf{x} \in \mathbb{R}^{n}$. Then for any $A \subset \mathbb{R}^{n}$,

$$
\mathbb{P}(\boldsymbol{X} \in A)=\sum_{\boldsymbol{x} \in A} f(\boldsymbol{x})
$$

If $\mathbf{X}$ is a continuous random vector, then the joint probability density function (pdf) of $\mathbf{X}$ is the function $f(\mathbf{x})=f\left(x_{1}, x_{2}, \ldots, x_{n}\right)$, with $\mathbf{x} \in \mathbb{R}^{n}$ that satisfies

$$
\mathbb{P}(\boldsymbol{X} \in A)=\int \cdots \int_{A} f(\boldsymbol{x}) \mathrm{d} \boldsymbol{x}
$$

for any $A \subset \mathbb{R}^{n}$. These integrals are $n$-fold integrals whose region of integration is over all points $\mathbf{x} \in A$.

Definition A.1.2 (Marginal Probability Distribution) (pg. 178 [1])

The marginal pmf or pdf of any subset of the coordinates of $\mathbf{X}=\left(X_{1}, X_{2}, \ldots, X_{n}\right)$ can be computed by summing or integrating the joint pmf or pdf over all possible values of the other coordinates. Thus the marginal distribution of $\left(X_{1}, X_{2}, \ldots, X_{k}\right)$, the first $k$ coordinates of $\mathbf{X}$, is given by the pmf or pdf

$$
f\left(x_{1}, x_{2}, \ldots, x_{k}\right)=\sum_{\left(x_{k+1}, \ldots, x_{n}\right) \in \mathbb{R}^{n-k}} f\left(x_{1}, x_{2}, \ldots, x_{n}\right)
$$


or

$$
f\left(x_{1}, x_{2}, \ldots, x_{k}\right)=\int_{-\infty}^{\infty} \cdots \int_{-\infty}^{\infty} f\left(x_{1}, x_{2}, \ldots, x_{n}\right) \mathrm{d} x_{k+1} \cdots \mathrm{d} x_{n}
$$

for every $\left(x_{1}, x_{2}, \ldots, x_{k}\right) \in \mathbb{R}^{k}$.

Definition A.1.3 (Mixture Distribution) (pg. 165 [1])

A random variable $X$ is said to have a mixture distribution if the distribution of $X$ depends on a quantity that also has a distribution.

Definition A.1.4 (Likelihood Function) (pg. $290[1]$ )

Let $f(\mathbf{x} \mid \theta)$ denote the joint pdf or pmf of the sample $\mathbf{X}=\left(X_{1}, X_{2}, \ldots, X_{n}\right)$. Then, given that $\mathbf{X}=\mathbf{x}$ is observed, the function of $\theta$ defined by

$$
L(\theta \mid \boldsymbol{x})=f(\boldsymbol{x} \mid \theta)
$$

is called the likelihood function. If $\mathbf{X}$ is a discrete random vector, then

$$
L(\theta \mid \boldsymbol{x})=\mathbb{P}_{\theta}(\boldsymbol{X}=\boldsymbol{x}) .
$$

Remark A.1.5 (Comparison of Possible Parameter Values) (pg. 290 [1])

Suppose $\mathbf{X}$ is a discrete random vector. If we compare the likelihood function at two parameter points and find that

$$
\mathbb{P}_{\theta_{1}}(\boldsymbol{X}=\boldsymbol{x})=L\left(\theta_{1} \mid \boldsymbol{x}\right)>L\left(\theta_{2} \mid \boldsymbol{x}\right)=\mathbb{P}_{\theta_{2}}(\boldsymbol{X}=\boldsymbol{x})
$$


then the sample we actually observed is more likely to have occurred if $\theta=\theta_{1}$ than if $\theta=\theta_{2}$, which can be interpreted as saying that $\theta_{1}$ is a more plausible value for the true value of $\theta$ than is $\theta_{2}$.

\section{Theorem A.1.6 (Likelihood Principle) (pg. 291 [1])}

If $\mathbf{x}$ and $\mathbf{y}$ are two sample points such that $L(\theta \mid \mathbf{x})$ is proportional to $L(\theta \mid \mathbf{y})$, that is, there exists a constant $C(\mathbf{x}, \mathbf{y})$ such that

$$
L(\theta \mid \boldsymbol{x})=C(\boldsymbol{x}, \boldsymbol{y}) L(\theta \mid \boldsymbol{y}) \quad \text { for all } \theta,
$$

then the conclusions drawn from $\mathbf{x}$ and $\mathbf{y}$ should be identical.

Definition A.1.7 (Maximum-Likelihood Estimator) (pg. 314 [1])

If $X_{1}, X_{2}, \ldots, X_{n}$ are an independent identically distributed sample from a population with pdf or pmf $f\left(x \mid \theta_{1}, \theta_{2}, \ldots, \theta_{k}\right)$, the likelihood function is defined by

$$
L(\theta \mid \boldsymbol{x})=L\left(\theta_{1}, \ldots, \theta_{k} \mid x_{1}, \ldots, x_{n}\right)=\prod_{i=1}^{n} f\left(x_{i} \mid \theta_{1}, \ldots, \theta_{k}\right) .
$$

For each sample point $\mathbf{x}$, let $\hat{\theta}(\mathbf{x})$ be a parameter value at which $L(\theta \mid \mathbf{x})$ attains its maximum as a function of $\theta$, with $\mathbf{x}$ held fixed. A maximum likelihood estimator (MLE) of the parameter $\theta$ based on a sample $\mathbf{X}$ is $\hat{\theta}(\mathbf{X})$. 
Definition A.1.8 (Maximum-Likelihood Function) (pg. 145 [21])

Let $\mathscr{F}$ be a class of probability distributions on $\Omega$. Then $F^{M L} \in \mathscr{F}$ will be called a maximum likelihood estimate of $F^{*}$ relative to $\mathscr{F}$ if

$$
L\left(F^{M L}\right) \geq L(F) \quad \text { for all } F \in \mathscr{F}
$$

In this case we write

$$
F^{M L}=\operatorname{argmax}\{L(F): F \in \mathscr{F}\}
$$

\section{Definition A.1.9 (Convergence in Probability) (pg. 54 [12])}

A sequence of random variables $Y_{n}$ defined over sample spaces $\left(\mathscr{Y}_{n}, \mathscr{B}_{n}\right)$ tends in probability to a constant $c$ if for every $\epsilon>0$

$$
\lim _{n \rightarrow \infty} \mathbb{P}\left(\left|Y_{n}-c\right| \geq \epsilon\right)=0 \quad \text { or } \quad Y_{n} \stackrel{\mathbb{P}}{\rightarrow} c
$$

Definition A.1.10 (Consistent Estimator) (pg. 54 [12])

A sequence of estimators $\delta_{n}$ of $g(\theta)$ is consistent if for every $\theta \in \Omega$ and for every $\epsilon>0$

$$
\lim _{n \rightarrow \infty} \mathbb{P}\left(\left|\delta_{n}-g(\theta)\right| \geq \epsilon\right)=0 \quad \text { or } \quad \delta_{n} \stackrel{\mathbb{P}_{\theta}}{\rightarrow} g(\theta)
$$




\section{A.2 Functional Analysis}

Definition A.2.1 (Hilbert Space) (pg. 77 [19])

If $H$ is a complete metric space, i.e. if every Cauchy sequence converges in $H$, then $H$ is called a Hilbert space.

Definition A.2.2 (Dual Space) (pg. 56 [20])

The dual space of a topological vector space $X$ is the vector space $X^{*}$ whose elements are the continuous linear functionals on $X$.

Definition A.2.3 (The C $(\Omega)$ Space) (pg. $33[20]$ )

If $\Omega$ is a nonempty open set in some euclidean space, then $\Omega$ is the union of countably many compact sets $K_{n} \neq \varnothing$ which can be chosen so that $K_{n}$ lies in the interior of $K_{n+1}(n=1,2, \ldots) . C(\Omega)$ is the vector space of all complexvalued continuous functions on $\Omega$.

Definition A.2.4 (The $\mathrm{C}^{\infty}(\Omega)$ Space) $\quad$ (pg. $\left.34[20]\right)$

A complex function $f$ defined in some nonempty open set $\Omega \subset \mathbb{R}^{n}$ is said to belong to $C^{\infty}(\Omega)$ if $D^{\alpha} f \in C(\Omega)$ for every multi-index $\alpha=\left(\alpha_{1}, \alpha_{2}, \ldots, \alpha_{n}\right)$ of non-negative integers. 
Definition A.2.5 (Support) (pg. $35[20]$ )

The support of a complex function $f$ (on any topological space) is the closure of $\{x: f(x) \neq 0\}$.

Definition A.2.6 (The $\mathscr{D}_{\mathbf{K}}$ Space) (pg. 35 [20])

If $K$ is a compact set in $\mathbb{R}^{n}$, then $\mathscr{D}_{K}$ denotes the space of all $f \in C^{\infty}\left(\mathbb{R}^{n}\right)$ whose support lies in $K$. If $K \subset \Omega$, then $\mathscr{D}_{K}$ may be identified with a subspace of $C^{\infty}(\Omega)$

Definition A.2.7 (Locally Integrable) (pg. $150[20]$ )

A complex function $f$ is said to be locally integrable if $f$ is measurable and $\int_{K}|f|<\infty$ for every compact $K \subset \mathbb{R}$. The idea is to reinterpret $f$ as being something that assigns the number $\int f \varphi$ to every suitably chosen "test function" $\varphi$, rather than as being something that assigns the number $f(x)$ to each $x \in \mathbb{R}$.

Definition A.2.8 (The $\mathscr{D}$ Space) (pg. $150[20]$ )

We let $\mathscr{D}=\mathscr{D}(\mathbb{R})$ be the vector space of all $\varphi \in C^{\infty}(\mathbb{R})$ whose support is compact. Then $\int f \varphi$ exists for every locally integrable $f$ and every $\varphi \in \mathscr{D}$. Moreover, $\mathscr{D}$ is sufficiently large to assure that $f$ is determined (a.e.) by the integrals $\int f \varphi$. 
Definition A.2.9 (The $\mathscr{D}(\Omega)$ Test Function Space) (pg. $151[20]$ )

Consider a nonempty open set $\Omega \subset \mathbb{R}^{n}$. The union of the spaces $\mathscr{D}_{K}$, as $K$ ranges over all compact subsets $\Omega$, is the test function space $\mathscr{D}(\Omega)$. It is clear that $\mathscr{D}(\Omega)$ is a vector space, with respect to the usual definitions of addition and scalar multiplication of complex functions. Explicitly, $\varphi \in \mathscr{D}(\Omega)$ if and only if $\varphi \in C^{\infty}(\Omega)$ and the support of $\varphi$ is a compact subset of $\Omega$. (See test function A.5.1.)

Definition A.2.10 (The $\tau$ Topology) (pg. $152[20]$ )

Let $\Omega$ be a nonempty open set in $\mathbb{R}^{n}$.

a) For every compact $K \subset \Omega, \tau_{K}$ denotes the Fréchet space topology of $\mathscr{D}_{K}$, as described in sections 1.46 and 6.2 (in [20]).

b) $\beta$ is the collection of all convex balanced sets $W \subset \mathscr{D}(\Omega)$ such that $\mathscr{D}_{K} \cap$ $W \in \tau_{K}$ for every compact $K \subset \Omega$.

c) $\tau$ is the collection of all unions of sets of the form $\varphi+W$, with $\varphi \in \mathscr{D}(\Omega)$ and $W \in \beta$.

Theorem A.2.11 (pg. $152[20]$ )

a) $\tau$ is a topology in $\mathscr{D}(\Omega)$, and $\beta$ is a local base for $\tau$.

b) $\tau$ makes $\mathscr{D}(\Omega)$ into a locally convex topological vector space. 
Definition A.2.12 (Distribution) (pg. $156[20]$ )

A linear functional on $\mathscr{D}(\Omega)$ which is continuous with respect to the topology $\tau$ (Definition A.2.10) is called a distribution in $\Omega$.

\section{Remark A.2.13 (Distribution Features) (pg. $149[20]$ )}

Here are some features that any such extension ought to have in order to be useful; our setting is some open subset of $\mathbb{R}^{n}$ :

a) Every continuous function should be a distribution.

b) Every distribution should have partial derivatives which are again distributions. For differentiable functions, the new motion of derivative should coincide with the old one. (Every distribution should therefore be infinitely differentiable.)

c) The usual formal rules of calculus should hold.

d) There should be a supply of convergence theorems that is adequate for handling the usual limit process.

Definition A.2.14 (The $\mathscr{D}^{\prime}(\Omega)$ Space) (pg. $156[20]$ )

The space of all distributions in $\Omega$ is denoted $\mathscr{D}^{\prime}(\Omega)$.

Definition A.2.15 (Support of a Distribution Functional) (pg. 164 [20])

Suppose $\Lambda \in \mathscr{D}^{\prime}(\Omega)$ is a functional. If $\omega$ is an open subset of $\Omega$ and if $\Lambda \varphi=0$ 
for every $\varphi \in \mathscr{D}(\omega)$, we say that $\Lambda$ vanishes in $\omega$. Let $W$ be the union of all open $\omega \subset \Omega$ in which $\Lambda$ vanishes. The complement of $W$ (relative to $\Omega$ ) is the support of $\Lambda$. 


\section{A.3 Convex Analysis}

Definition A.3.1 (Convex Function) (pg. 2 [18])

A function $f: I \rightarrow \mathbb{R}$ is called convex if

$$
f[\lambda x+(1-\lambda) y] \leq \lambda f(x)+(1-\lambda) f(y)
$$

for all $x, y \in I$ and $\lambda \in(0,1)$.

Definition A.3.2 (Strictly Convex Function) (pg. 2 [18])

A function $f: I \rightarrow \mathbb{R}$ is called strictly convex provided that

$$
f[\lambda x+(1-\lambda) y]<\lambda f(x)+(1-\lambda) f(y)
$$

for all $x, y \in I, x \neq y$, and $\lambda \in(0,1)$.

Definition A.3.3 (Concave Function) (pg. 2 [18])

If $-f: I \rightarrow \mathbb{R}$ is convex, then we say $f: I \rightarrow \mathbb{R}$ is concave.

Definition A.3.4 (Linear Function) (pg. 2 [18])

We say $f: \mathbb{R} \rightarrow \mathbb{R}$ is linear if

$$
f(\alpha x+\beta y)=\alpha f(x)+\beta f(y)
$$

for all $\alpha, \beta, x, y \in \mathbb{R}$. It is also known and is easy to show that $f$ is linear if and only if $f(x)=m x$ for some constant $m$. 
Definition A.3.5 (Affine Function) (pg. 2 [18])

We say that $f: I \rightarrow \mathbb{R}$ is affine if it is of the form $f(x)=m x+b$ on $I$. It is clear that any affine function is convex, but not strictly convex.

Theorem A.3.6 (pg. 10 [18])

Suppose $f$ is differentiable on $(a, b)$. Then $f$ is convex [strictly convex] if and only if $f^{\prime}$ is increasing [strictly increasing].

Definition A.3.7 (Support Point) (pg. $12[18]$ )

We say that a function $f$ defined on $I$ has support at $x_{0} \in I$ if there exists an affine function $A(x)=f\left(x_{0}\right)+m\left(x-x_{0}\right)$ such that $A(x) \leq f(x)$ for every $x \in I$. The graph of the support function $A$ is called a line of support for $f$ at $x_{0}$.

Theorem A.3.8 (pg. 15 [18])

If $f: I \rightarrow \mathbb{R}$ and $g: I \rightarrow \mathbb{R}$ are convex and $\alpha \geq 0$, then $f+g$ and $\alpha f$ are convex on $I$.

Theorem A.3.9 (pg. $16[18]$ )

Let $f: I \rightarrow \mathbb{R}$ and $g: J \rightarrow \mathbb{R}$ where $f(I) \subseteq J$. If $f$ and $g$ are convex and $g$ is increasing, then the composite function $g \circ f$ is convex on $I$. 
Theorem A.3.10 (pg. 16 [18])

If $f: I \rightarrow \mathbb{R}$ and $g: I \rightarrow \mathbb{R}$ are both non-negative, decreasing [increasing], and convex, then $h(x)=f(x) g(x)$ also exhibits these properties.

Definition A.3.1 1 (Convex Set) (pg. 73 [18])

Let $U$ be a subset of a linear space $\mathbf{L}$. We say that $U$ is convex if $\mathbf{x}, \mathbf{y} \in U$ implies that $\mathbf{z}=[\lambda x+(1-\lambda) y] \in U$ for all $\lambda \in[0,1]$.

Definition A.3.12 (Affine Set) (pg. 73 [18])

$U$ is affine if $\mathbf{x}, \mathbf{y} \in U$ implies that $\mathbf{z}=[\lambda x+(1-\lambda) y] \in U$ for all $\lambda \in \mathbb{R}$.

Definition A.3.13 (Affine Combination) (pg. 75 [18])

Let $\mathbf{L}$ be a linear space. If $\lambda_{i} \in \mathbb{R}$ and $\sum_{i=1}^{n} \lambda_{i}=1$, then

$$
\boldsymbol{x}=\sum_{i=1}^{n} \lambda_{i} x_{i}
$$

is called an affine combination of $\mathbf{x}_{1}, \mathbf{x}_{2}, \ldots, \mathbf{x}_{n}$, the latter being elements of $\mathbf{L}$.

Definition A.3.14 (Convex Combination) (pg. 75 [18])

In Definition A.3.13, if in addition $\lambda_{i} \geq 0$ for all $i$, then

$$
\boldsymbol{x}=\sum_{i=1}^{n} \lambda_{i} x_{i}
$$

is called a convex combination of $\mathbf{x}_{1}, \mathbf{x}_{2}, \ldots, \mathbf{x}_{n}$. 
Theorem A.3.15 (pg. 75 [18])

Let $\mathbf{L}$ be a linear space. A set $U \subseteq \mathbf{L}$ is convex [affine] if and only if every convex [affine] combination of points of $U$ lies in $U$.

Definition A.3.16 (Convex Hull) (pg. 75 [18])

We call the intersection of all convex sets containing a given set $U$ the convex hull of $U$, denoted by $H(U)$.

Definition A.3.17 (Affine Hull) (pg. 75 [18])

We call the intersection of all affine sets containing a given set $U$ the affine hull of $U$.

Theorem A.3.18 (pg. 76 [18])

Let $\mathbf{L}$ be a linear space. For any $U \subseteq \mathbf{L}$, the convex [affine] hull of $U$ consists precisely of all convex [affine] combinations of elements of $U$.

Theorem A.3.19 (Carathéodory Theorem) (pg. 76 [18])

Let $\mathbf{L}$ be a linear space. If $U \subseteq \mathbf{L}$ and its convex hull $H(U)$ has dimension $m$, then for each $\mathbf{z} \in H(U)$, there exists $m+1$ points $\mathbf{x}_{0}, \mathbf{x}_{1}, \ldots, \mathbf{x}_{m}$ of $U$ such that $\mathbf{z}$ is a convex combination of these points. 
Definition A.3.20 (Mixture Density) (pg. 86 [13])

Let $\left\{f_{\theta}: \theta \in \Omega\right\}$ be a parametric family of densities with respect to some sigmafinite measure. Let the parameter space $\Omega$ have a sigma field of measurable sets which contains all atomic sets $\{\theta\}$. Let $\mathscr{M}$ be the class of all probability measures on $\Omega$. Define the function

$$
f_{Q}(x)=\int f_{\theta}(x) \mathrm{d} Q(\theta), \quad Q \in \mathscr{M}
$$

to be the mixture density corresponding to mixing distribution $Q$.

Definition A.3.21 (Atomic Density) (pg. 86 [13])

Let $M \subseteq \Omega$. Since the densities $f_{\theta}$ correspond to the atomic mixing distributions

$$
\delta(\theta)= \begin{cases}1, & \text { if } \quad \theta \in M \\ 0, & \text { else }\end{cases}
$$

which assign probability one to any set $\operatorname{containing} \theta$, they are called the atomic densities.

Definition A.3.22 (Likelihood Curve) (pg. 87 [13])

Suppose the observation vector $\left(x_{1}, x_{2}, \ldots, x_{n}\right)$ has $K$ distinct data points $y_{1}, y_{2}, \ldots, y_{K}$. Let $n_{k}$ be the number of $x$ 's which equal $y_{k}$. Define the atomic and mixture 
likelihood vectors to be $f_{\theta}=\left(f_{\theta}\left(y_{1}\right), \ldots, f_{\theta}\left(y_{K}\right)\right)$ and $f_{Q}=\left(f_{Q}\left(y_{1}\right), \ldots, f_{Q}\left(y_{K}\right)\right)$ respectively. The likelihood curve is the function from $\Omega$ to $\mathbb{R}$ defined by $\theta \rightarrow f_{\theta}$. The trace of this curve, given by $\Gamma=\left\{f_{\theta}: \theta \in \Omega\right\}$, represents all possible fitted values of the atomic likelihood vector.

\section{Theorem A.3.23 (Existence of Finite Support) (pg. 89 [13])}

If $\Gamma$, the likelihood curve, as in Definition A.3.22, is closed and bounded (compact), then there exists a unique vector $\hat{f}$ on the boundary of the convex hull, $H(\Gamma)$, which maximizes the log likelihood $\phi(\cdot)$ on that set. The point $\hat{f}$ can be expressed as $f_{Q}$, where $Q$ has $K$ or fewer points of support.

Definition A.3.24 (Heaviside Function) (pg. 335 [22])

The Heaviside function (or step function) is defined by

$$
H(x)=\left\{\begin{array}{lcc}
1 & \text { if } \quad x \geq 0, \\
0 & \text { if } \quad x<0 .
\end{array} .\right.
$$

For any test function,

$$
\left(H^{\prime}, \phi\right)=-\left(H, \phi^{\prime}\right)=-\int_{-\infty}^{\infty} H(x) \phi^{\prime}(x) \mathrm{d} x=-\int_{0}^{\infty} \phi^{\prime}(x) \mathrm{d} x=-\left.\phi(x)\right|_{0} ^{\infty}=\phi(0) .
$$


Thus

$$
H^{\prime}=\delta
$$

Definition A.3.25 (Primal Problem) (pg. 127, 223 [2])

We use the notation

$$
\begin{aligned}
& \text { minimize } f_{0}(x) \\
& \text { subject to } \quad f_{i}(x) \leq 0, \quad i=1,2, \ldots, m \\
& h_{i}(x)=0, \quad i=1,2, \ldots, p
\end{aligned}
$$

to describe the problem of finding an $x$ that minimizes $f_{0}(x)$ among all $x$ that satisfy the conditions $f_{i}(x) \leq 0, i=1,2, \ldots, m$ and $h_{i}(x)=0, i=1,2, \ldots, p$. We call $x \in \mathbb{R}^{n}$ the optimization variable and the function $f_{0}: \mathbb{R}^{n} \rightarrow \mathbb{R}$ the objective function or cost function. The inequalities $f_{i}(x) \leq 0$ are called inequality constraints and the corresponding functions $f_{i}: \mathbb{R}^{n} \rightarrow \mathbb{R}$ are called the inequality constraint functions. The equations $h_{i}(x)=0$ are called equality constraints and the corresponding functions $h_{i}: \mathbb{R}^{n} \rightarrow \mathbb{R}$ are called the equality constraint functions. If there are no constraints (i.e. $m=p=0$ ) we say the problem in Equation (3.25) is unconstrained. This is the standard form of the optimization problem. In this context, the problem is sometimes called the primal problem. 
Definition A.3.26 (Domain of the Optimization Problem) (pg. 127 [2])

The set of points for which the objective and all constraint functions are defined,

$$
\mathscr{D}=\bigcap_{i=0}^{m} \operatorname{dom}\left(f_{i}\right) \cap \bigcap_{i=0}^{p} \operatorname{dom}\left(h_{i}\right),
$$

is called the domain of the optimization problem.

Definition A.3.27 (Feasible) (pg. 127 [2])

A point $x \in \mathscr{D}$ is feasible if it satisfies the constraints $f_{i}(x) \leq 0, i=1,2, \ldots, m$ and $h_{i}(x)=0, i=1,2, \ldots, p$. The problem in Definition A.3.25 is said to be feasible if there exists at least one feasible point, and infeasible otherwise. The set of all feasible points is called the feasible set or constraint set.

Definition A.3.28 (Optimal Value) (pg. 127 [2])

A optimal value $p^{*}$ of the problem in Definition A.3.25 is defined as

$$
p^{*}=\inf \left\{f_{0}(x) \mid f_{i}(x) \leq 0, i=1,2, \ldots, m, h_{i}(x)=0, i=1,2, \ldots, p\right\}
$$

We allow $p^{*}$ to take on the extended values $\pm \infty$. If the problem is infeasible, we have $p^{*}=\infty$ (following the standard convention that the infimum of the empty set is $\infty$ ). If there are feasible points $x_{k}$ with $f\left(x_{k}\right) \rightarrow-\infty$ as $k \rightarrow \infty$, then $p^{*}=-\infty$, and we say the problem in Definition A.3.25 is unbounded below. 
Definition A.3.29 (Optimal Point) (pg. $128[2]$ )

We say $x^{*}$ is an optimal point, or solves the problem in Definition A.3.25 if $x^{*}$ is feasible and $f_{0}\left(x^{*}\right)=p^{*}$. The set of all optimal points is the optimal set, denoted

$$
X_{o p t}=\left\{x \mid f_{i}(x) \leq 0, i=1,2, \ldots, m, h_{i}(x)=0, i=1,2, \ldots, p, f_{0}(x)=p^{*}\right\}
$$

If there exists an optimal point for the problem in Definition A.3.25, we say the optimal value is attained or achieved, and the problem is solvable.

Theorem A.3.30 (Equivalent Problems) (pg. 130 [2])

We call two problems equivalent if from a solution of one, a solution of the other is readily found, and visa versa.

Definition A.3.31 (Convex Optimization Problem) (pg. 136 [2])

A convex optimization problem is one of the form

$$
\begin{aligned}
& \text { minimize } f_{0}(x) \\
& \text { subject to } \quad f_{i}(x) \leq 0, \quad i=1,2, \ldots, m \\
& a_{i}^{T} x=b_{i}, \quad i=1,2, \ldots, p
\end{aligned}
$$

where $f_{0}, f_{1}, \ldots, f_{m}$ are convex functions. Comparing this with the standard form problem in Definition A.3.25, the convex problem has three additional requirements 
- the objective function must be convex,

- the inequality constraint functions must be convex,

- the equality constraint functions $h_{i}(x)=a_{i}^{T} x-b_{i}$ must be affine.

Definition A.3.32 (Feasible Set and Domain of the Convex Problem) (pg. 137 [2])

The feasible set of a convex optimization problem is convex, since it is the intersection of the domain of the problem

$$
\mathscr{D}=\bigcap_{i=0}^{m} \operatorname{dom}\left(f_{i}\right)
$$

which is a convex set, with $m$ (convex) sublevel sets $\left\{x \mid f_{i}(x) \leq 0\right\}$ and $p$ hyperplanes $\left\{x \mid a_{i}^{T} x=b_{i}\right\}$. Thus, in a convex optimization problem, we minimize a convex objective function over a convex set.

\section{Theorem A.3.33 (Local and Global Optimal Point) (pg. 138 [2])}

A fundamental property of convex optimization problems is that any locally optimal point is also globally optimal.

Definition A.3.34 (Lagrangian) (pg. 215 [2])

We consider an optimization problem in the standard form (see Definition A.3.25) with $x \in \mathbb{R}^{n}$. We do not assume that the problem is convex. We define 
the Lagrangian $L: \mathbb{R}^{n} \times \mathbb{R}^{m} \times \mathbb{R}^{p} \rightarrow \mathbb{R}$ as

$$
L(x, \lambda, v)=f_{0}(x)+\sum_{i=1}^{m} \lambda_{i} f_{i}(x)+\sum_{i=1}^{p} v_{i} h_{i}(x)
$$

with $\operatorname{dom}(L)=\mathscr{D} \times \mathbb{R}^{m} \times \mathbb{R}^{p}$. We refer to $\lambda_{i}$ as the Lagrange multiplier associated with the $i^{\text {th }}$ inequality constraint $f_{i}(x) \leq 0$; similarly we refer to $v_{i}$ as the Lagrange multiplier associated with the $i^{\text {th }}$ equality constraint $h_{i}(x)=0$. The vectors $\lambda$ and $v$ are called the dual variables or Lagrange multiplier vectors associated with the problem.

Definition A.3.35 (Lagrangian Dual Function) (pg. 216 [2])

We define the Lagrange dual function (or just dual function) $g: \mathbb{R}^{m} \times \mathbb{R}^{p} \rightarrow \mathbb{R}$ as the minimum value of the Lagrangian over $\mathrm{x}$ : for $\lambda \in \mathbb{R}^{n}, v \in \mathbb{R}^{p}$,

$$
g(\lambda, v)=\inf _{x \in \mathscr{D}} L(x, \lambda, v)
$$

\section{Definition A.3.36 (Lagrangian Dual Problem) (pg. 223 [2])}

For each pair $(\lambda, v)$ with $\lambda \geq 0$, the Lagrangian dual function gives us a lower bound on the optimal value $p^{*}$ of the optimization problem in Definition A.3.25. Thus we have a lower bound that depends on some parameters $\lambda, v$. A natural question is: What is the best lower bound that can be obtained from 
the Lagrangian dual function? This leads to the problem

$$
\begin{array}{cl}
\text { maximize } & g(\lambda, v) \\
\text { subject to } & \lambda \geq 0 .
\end{array}
$$

This problem is called the Lagrangian dual problem associated with the problem in Definition A.3.25.

Definition A.3.37 (Dual Feasible) (pg. 223 [2])

The term dual feasible, to describe the pair $(\lambda, v)$ with $\lambda \geq 0$ and $g(\lambda, v)>-\infty$, now makes sense. It means, as the name implies, that $(\lambda, v)$ is feasible for the dual problem in Definition A.3.34. We refer to $\left(\lambda^{*}, v^{*}\right)$ as dual optimal or optimal Lagrangian multipliers if they are optimal for the problem. The Lagrange dual problem is a convex optimization problem, since the objective to be maximized is concave and the constraint is convex. This is the case whether or not the primal problem in Definition A.3.25 is convex.

Definition A.3.38 (Weak Duality) (pg. 225 [2])

The optimal value of the Lagrange dual problem, which we denote $d^{*}$, is by definition, the best lower bound on $p^{*}$ that can be obtained from the Lagrange dual function. In particular, we have the simple but important inequality

$$
d^{*} \leq p^{*}
$$


which holds even if the original problem is not convex. This property is called weak duality. The weak duality inequality holds when $d^{*}$ and $p^{*}$ are infinite. For example, if the primal problem is unbounded below, so that $p^{*}=-\infty$, we must have $d^{*}=-\infty$, i.e. the Lagrange dual problem is infeasible. Conversely, if the dual problem is unbounded above, so that $d^{*}=\infty$, we must have $p^{*}=$ $\infty$, i.e. the primal problem is infeasible.

\section{Definition A.3.39 (Optimal Duality Gap) (pg. 226 [2])}

We refer to the difference $d^{*}-p^{*}$ as the optimal duality gap of the original problem, since it gives the gap between the optimal value of the primal problem and the best (i.e. greatest) lower bound on it that can be obtained from the Lagrangian dual function. The optimal duality gap is always nonnegative.

\section{Definition A.3.40 (Karush-Kuhn-Tucker Conditions) (pg. 243 [2])}

Assume that the functions $f_{0}, f_{1}, \ldots, f_{m}, h_{1}, h_{2}, \ldots, h_{p}$ are differentiable (and therefore have open domains), but we make no assumptions yet about convexity. Let $x^{*}$ and $\left(\lambda^{*}, v^{*}\right)$ be any dual optimal points with zero duality gap. Since $x^{*}$ minimizes $L\left(x, \lambda^{*}, v^{*}\right)$ over $x$, it follows that its gradient must vanish at $x^{*}$, i.e.,

$$
\nabla f_{0}\left(x^{*}\right)+\sum_{i=1}^{m} \lambda_{i}^{*} \nabla f_{i}\left(x^{*}\right)+\sum_{i=1}^{p} v_{i}^{*} \nabla h_{i}\left(x^{*}\right)=0
$$


Thus we have

$$
\begin{aligned}
& f_{i}\left(x^{*}\right) \leq 0, \quad i=1,2, \ldots, m \\
& h_{i}\left(x^{*}\right)=0, \quad i=1,2, \ldots, p \\
& \lambda_{i}^{*} \geq 0, \quad i=1,2, \ldots, m \\
& \lambda_{i}^{*} f_{i}\left(x^{*}\right)=0, \quad i=1,2, \ldots, m \\
& \nabla f_{0}\left(x^{*}\right)+\sum_{i=1}^{m} \lambda_{i}^{*} \nabla f_{i}\left(x^{*}\right)+\sum_{i=1}^{p} v_{i}^{*} \nabla h_{i}\left(x^{*}\right)=0,
\end{aligned}
$$

which are called the Karush-Kuhn-Tucker (KKT) conditions. To summarize, for any optimization problem with differentiable objective and constraint functions for which strong duality obtains, any pair of primal and dual optimal points must satisfy the KKT conditions.

Definition A.3.41 (Convex KKT Conditions) (pg. 243 [2])

When the primal problem is convex, the KKT conditions are also sufficient for the points to be primal and dual optimal. In other words, if $f_{i}$ are convex and 
$h_{i}$ are affine, and $\bar{x}, \bar{\lambda}, \bar{v}$ are any points that satisfy the KKT conditions

$$
\begin{array}{rlrl}
f_{i}(\bar{x}) & \leq 0, & i=1,2, \ldots, m \\
h_{i}(\bar{x}) & =0, & i=1,2, \ldots, p \\
\overline{\lambda_{i}} \geq 0, & i=1,2, \ldots, m \\
\overline{\lambda_{i}} f_{i}(\bar{x}) & =0, & i=1,2, \ldots, m \\
\nabla f_{0}(\bar{x})+\sum_{i=1}^{m} \overline{\lambda_{i}} \nabla f_{i}(\bar{x})+\sum_{i=1}^{p} \overline{v_{i}} \nabla h_{i}(\bar{x}) & =0, &
\end{array}
$$

then $\bar{x}$ and $(\bar{\lambda}, \bar{v})$ are primal and dual optimal, with zero duality gap.

\section{Definition A.3.42 (Primal Problem in LP) (pg. 2 [24])}

Let the optimization problem be a linear programming problem and thus a convex optimization problem. The standard form (primal problem) is

$$
\min c^{T} x \text { subject to } A x=b, x \geq 0,
$$

where $c$ and $x$ are vector in $\mathbb{R}^{n}, b$ is a vector in $\mathbb{R}^{m}$, and $A$ is an $m \times n$ matrix.

\section{Definition A.3.43 (Dual Problem in LP) (pg. 3 [24])}

The dual problem is:

$$
\max b^{T} \lambda \text { subject to } A^{T} \lambda+s=c, s \geq 0,
$$

where $\lambda$ is a vector in $\mathbb{R}^{m}$ and $s$ is a vector in $\mathbb{R}^{n}$. The vector $s$ is called the dual slacks. 
Theorem A.3.44 (pg. 4 [24])

The vector $x^{*} \in \mathbb{R}^{n}$ is a solution of Equation 3.51 if and only if there exist vectors $s^{*} \in \mathbb{R}^{n}$ and $\lambda^{*} \in \mathbb{R}^{m}$ for which the following conditions hold for $(x, \lambda, s)=$ $\left(x^{*}, \lambda^{*}, s^{*}\right):$

$$
\begin{aligned}
A^{T} \lambda+s & =c, \\
A x & =b, \\
x_{i} s_{i} & =0, \quad i=1,2, \ldots, n \\
(x, s) & \geq 0 .
\end{aligned}
$$

(Note: in this construction, $\lambda$ and $s$ are the Lagrange multipliers for the constraints $A x=b$ and $x \geq 0$, respectively.)

Theorem A.3.45 (pg. 4 [24])

The vector $\left(\lambda^{*}, s^{*}\right) \in \mathbb{R}^{m} \times \mathbb{R}^{n}$ is a solution of Equation 3.52 if and only if there exists a vector $x^{*} \in \mathbb{R}^{n}$ such that the conditions in Equations 3.53 through 3.56 hold for $(x, \lambda, s)=\left(x^{*}, \lambda^{*}, s^{*}\right)$.

Definition A.3.46 (Primal-Dual Method in LP) (pg. 4 [24])

Examining the conditions in Equations 3.53 through 3.56 from both the primal viewpoint in Definition A.3.42 and the dual viewpoint in Definition A.3.43, 
we can conclude that a vector $\left(x^{*}, \lambda^{*}, s^{*}\right)$ solve the system if and only if $x^{*}$ solves the primal problem and $\left(\lambda^{*}, s^{*}\right)$ solves the dual problem. The vector $\left(x^{*}, \lambda^{*}, s^{*}\right)$ is called a primal-dual solution.

\section{Definition A.3.47 (Interior-Point Method in LP) (pg. 4, 5 [24])}

Let us restate the optimality conditions in Equations 3.53 through 3.56 in a slightly different form by means of a mapping $F$ from $\mathbb{R}^{2 n+m}$ to $\mathbb{R}^{2 n+m}$ :

$$
\begin{gathered}
F(x, \lambda, s)=\left[\begin{array}{c}
A^{T} \lambda+s-c \\
A x-b \\
X S e
\end{array}\right]=0 \\
(x, s) \geq 0
\end{gathered}
$$

where

$$
\begin{aligned}
& X=\operatorname{diag}\left(x_{1}, x_{2}, \ldots, x_{n}\right) \\
& S=\operatorname{diag}\left(s_{1}, s_{2}, \ldots, s_{n}\right) \\
& e=(1,1, \ldots, 1)^{T} .
\end{aligned}
$$

Note that $F$ is actually linear in its first two terms and only mildly nonlinear in the remaining term. All primal-dual methods generate iterates $\left(x^{k}, \lambda^{k}, s^{k}\right)$ that satisfy the bounds in Equation 3.58 strictly, that is $x^{k}>0$ and $s^{k}>0$. This 
property is the origin of the term interior-point. By respecting these bounds, the methods avoid spurious solutions, which are points that satisfy $F(x, \lambda, s)=$ 0 but not $(x, s) \geq 0$.

Remark A.3.48 (Primal-Dual Interior-Point Method) (pg. 5 [24])

Like most iterative algorithms in optimization, primal-dual interior-point methods have two basic ingredients:

- a procedure for determining the step

- a measure of the desirability of each point in the search space.

The search direction procedure has its origins in Newton's method for the nonlinear equations in Equation 3.57. 


\section{A.4 Differential Equations}

\section{Definition A.4.1 (Heat Equation) (pg. 16 [22])}

Let $u(x, y, z, t)$ be the temperature and let $H(t)$ be the amount of heat contained in region $D$. Then

$$
H(t)=\iiint_{D} c \rho u \mathrm{~d} x \mathrm{~d} y \mathrm{~d} z
$$

where $c$ is the specific heat of the material and $\rho$ is the density (mass per unit volume). The change in heat is

$$
\frac{\mathrm{d} H}{\mathrm{~d} t}=\iiint_{D} c \rho u_{t} \mathrm{~d} x \mathrm{~d} y \mathrm{~d} z
$$

Fourier's law says that the heat flows from hot to cold regions proportionally to the temperature gradient. But the heat cannot be lost from $D$ except by leaving through the boundary. This is law of conservation of energy. Therefore, the change in energy in $D$ also equals the heat flux across the boundary,

$$
\frac{\mathrm{d} H}{\mathrm{~d} t}=\iint_{\partial D} \kappa(\mathbf{n} \cdot \nabla u) \mathrm{d} S,
$$

where $\kappa$ is a proportionality factor (heat conductivity). By the divergence theorem,

$$
\iiint_{D} c \rho \frac{\partial u}{\partial t} \mathrm{~d} x \mathrm{~d} y \mathrm{~d} z=\iiint_{D} \nabla \cdot(\kappa \nabla u) \mathrm{d} x \mathrm{~d} y \mathrm{~d} z
$$


and we get the heat equation

$$
c \rho \frac{\partial u}{\partial t}=\nabla \cdot(\kappa \nabla u)
$$

If $c, \rho$, and $\kappa$ are constants, it is exactly the same as the diffusion equation. Note: In this paper, $c, \rho$, and $\kappa$ are constant and we are solely concerned with the depth so the heat equation does reduce

$$
\frac{\partial u}{\partial t}=\frac{\kappa}{c \rho} \nabla^{2} u
$$

to

$$
\frac{\partial u}{\partial t}(z, t)=q \frac{\partial^{2} u}{\partial z^{2}}(z, t)
$$

where $q=\frac{\kappa}{c \rho}$.

Definition A.4.2 (Classical Solution) (pg. 5 [16])

By a classical solution we mean a sufficiently smooth function $u$ of the independent variables that satisfies the differential equation at every point of its domain of definition. We do not necessarily require that the solution be defined for all possible values of the independent variables. Indeed, usually the differential equation is imposed on some domain $D$ contained in the space of independent variables, and we seek a solution defined only on $D$. In general, the domain $D$ will be an open subset, usually connected and, particularly in 
equilibrium equations, often bounded, with a reasonably nice boundary, denoted by $\partial D$.

Lemma A.4.3 (pg. $428[16])$

Let $V$ be an inner product space with inner product $\langle\cdot, \cdot\rangle$. An element $v_{*} \in V$ satisfies $\left\langle\left\langle v_{*}, v\right\rangle\right\rangle=0$ for all $\nu \in V$ if and only if $\nu_{*}=0$.

Definition A.4.4 (Weak Formulation) (pg. 428 [16])

Let $F: U \rightarrow V$ be a linear or nonlinear system. One method to solve $F[u]=0$, see Lemma A.4.3, is to write it in the form

$$
\langle\langle F[u], v\rangle\rangle=0 \text {, for all } v \in V \text {. }
$$

In particular, for an inhomogeneous linear system, $L[u]=f$, with $L: U \rightarrow V$ a linear operator between inner product spaces, the condition in Equation (4.64) takes the form

$$
0=\langle\langle L[u]-f, v\rangle\rangle=\langle\langle L[u], v\rangle\rangle-\langle\langle f, v\rangle\rangle \text {, for all } v \in V
$$

or, equivalently,

$$
\left\langle u, L^{*}[u]\right\rangle-\langle\langle f, v\rangle\rangle, \text { for all } v \in V
$$

where $L^{*}: V \rightarrow U$ denotes the adjoint of the operator $L$. We will call Equation (4.66) the weak formulation of the original linear system. Note: Thanks to 
the integration by parts argument to determine the adjoint operator, the solution $u$ to the weak form in Equation (4.66) is not restricted by the degree of smoothness required of a classical solution (see Definition A.4.2).

Definition A.4.5 (Weak Solution) (pg. $428[16])$

Suppose an arbitrary smooth test function $v$ satisfies the boundary condition on a function $u$ and the integral of the product of this test function with equation in the original system converges in the weak topology. A function $u$ that satisfies this integral condition is called the weak solution to the original boundary value problem. 


\section{A.5 Numerical Analysis}

Definition A.5.1 (Test Function) (pg. 331, 336 [22])

A test function $\varphi(\mathbf{x})=\varphi\left(x_{1}, x_{2}, \ldots, x_{n}\right)$ is a real $C^{\infty}$ function that vanishes outside some ball (interval of support A.2.5). (See test function space A.2.9.)

Definition A.5.2 (Spline) (pg. 58 [17])

A curve $s(u)$ is called a spline of degree $n$ with knots $a_{0}, a_{1}, \ldots, a_{m}$, where $a_{i} \leq$ $a_{i+1}$ and $a_{i}<a_{i+n+1}$ for all possible $i$ if $s(u)$ is $n-r$ times differentiable at any $r$-fold knot, and $s(u)$ is a polynomial of degree $\leq n$ over each knot interval $\left[a_{i}, a_{i+1}\right]$, for $i=0,1, \ldots, m-1$. It is also common to refer to a spline of degree $n$ as a spline of order $n+1$.

(Note: A knot $a_{i+1}$ is called $\mathbf{r}$-fold if $a_{i}<a_{i+1}=\cdots=a_{i+r}<a_{i+r+1}$ )

Definition A.5.3 (B-Spline) (pg. 58,61 [17])

As with the Bézier representation of polynomial curves, it is desirable to write a spline $s(u)$ as an affine combination (see A.3.13) of some control points $c_{i}$, namely

$$
s(u)=\sum c_{i} N_{i}^{n}(u)
$$

where the $N_{i}^{n}(u)$ are basis spline (B-spline) functions with minimal support and certain continuity properties. 
To define B-splines, let $\left(a_{i}\right)$ be, for simplicity, a bi-infinite and strictly increasing sequence of knots (which means $a_{i}<a_{i+1}$, for all $i$ ). We define the B-splines $N_{i}^{n}$ with these knots by the recursion formula

$$
N_{i}^{0}(u)=\left\{\begin{array}{l}
1 \text { if } u \in\left[a_{i}, a_{i+1}\right) \\
0 \text { otherwise }
\end{array}\right.
$$

and

$$
N_{i}^{n}(u)=\alpha_{i}^{n-1} N_{i}^{n-1}(u)+\left(1-\alpha_{i+1}^{n-1}\right) N_{i+1}^{n-1}(u),
$$

where

$$
\alpha_{i}^{n-1}=\frac{u-a_{i}}{a_{i+n}-a_{i}}
$$

is the local parameter with respect to the support of $N_{i}^{n-1}$. The following properties of B-splines are evident:

(i) $N_{i}^{n}$ is a piecewise polynomial of degree $n$.

(ii) $N_{i}^{n}$ is positive in $\left(a_{i}, a_{i+n+1}\right)$.

(iii) $N_{i}^{n}$ is zero outside of $\left[a_{i}, a_{i+n+1}\right]$.

(iv) $N_{i}^{n}$ is right side continuous. 
Definition A.5.4 (Galerkin Method) (pg. $635[10]$ )

Suppose we are confronted with a problem of the form

$$
L u=f
$$

in which $L$ is a linear operator, $f$ is a given function, and $u$ is a function to be determined from the equation. In the Galerkin method, we select a set of basic functions or test functions $u_{1}, u_{2}, \ldots, u_{n}$. We then attempt to solve Equation (5.71) with a suitable linear combination of these basic functions. Setting

$$
u=\sum_{j=1}^{n} c_{j} u_{j}
$$

and using the linearity of $L$, we obtain

$$
\sum_{j=1}^{n} c_{j} L u_{j}=f
$$

In a typical situation, this equation is inconsistent because $f$ does generally not lie in the vector space spanned by the functions $L u_{j}$. We therefore solve Equation (5.73) approximately and obtain thereby an approximate solution to Equation (5.71). The approximate solving of Equation (5.73) can be carried out according to many different criteria, each of which leads to a different approximate solution $u$. 
The most natural approach is to select $c_{1}, c_{2}, \ldots, c_{n}$ so that some norm is minimized:

$$
\left\|\sum_{j=1}^{n} c_{j} L u_{j}-f\right\|=\min .
$$

This is a problem in best approximation. We are approximating $f$ by the nearest element in the subspace generated by the functions $L u_{j}$. This is relatively easy in an inner-product space because the technique of orthogonal projection is available.

\section{Definition A.5.5 (Collocation) (pg. $635[10])$}

A very general way of obtaining an approximate solution of Equation (5.73) is to select a set of linear functionals $\varphi_{1}, \varphi_{2}, \ldots, \varphi_{n}$ and impose the condition

$$
\varphi_{i}\left(\sum_{j=1}^{n} c_{j} L u_{j}-f\right)=0,(1 \leq i \leq n)
$$

By linearity of the functionals, this becomes

$$
\sum_{j=1}^{n} c_{j} \varphi_{i}\left(L u_{j}\right)=\varphi_{i}(f),(1 \leq i \leq n)
$$

Equation (5.76) is a system of $n$ linear equations in the $n$ unknowns $c_{j}$. If the functionals are point-evaluation functionals, defined by

$$
\varphi_{i}(v)=v\left(x_{i}\right),(1 \leq i \leq n)
$$

then the method above is called collocation. 
Definition A.5.6 (Classical Galerkin Method) (pg. 636 [10])

The classical Galerkin method is a particular case of Equation (5.76) in a Hilbert space, where $\varphi_{i}(v)=\left\langle u_{i}, v\right\rangle$. Thus the equations to be solved in this case are

$$
\sum_{j=1}^{n} c_{j}\left\langle u_{i}, L u_{j}\right\rangle=\left\langle u_{i}, f\right\rangle,(1 \leq i \leq n)
$$

Definition A.5.7 (Finite Element Method) (pg. 641 [10])

When the Galerkin method (see Definition A.5.4) employs basic (test) functions that are piecewise polynomials, the procedure is called the finite-element method.

(Note: B-splines (see A.5.3) are the basic functions used in the FEM in Chapter 3.)

(Note: This is the method used in Chapter 3 of this thesis to numerically approximate alcohol concentration values.)

Definition A.5.8 (Faure Grid) $\quad$ (p. $363[5])$

A Faure grid is constructed from two quasirandom Faure sequences. This grid has increased properties of accuracy, low discrepancy, and speed as opposed to a standard uniform grid when used in global optimization. 


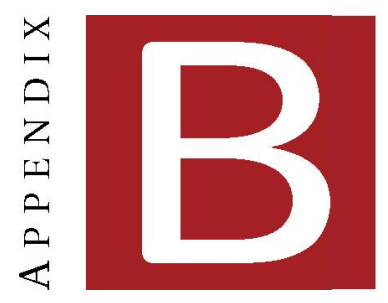

\section{ALGORITHMS}

This appendix outlines several algorithms for the tasks of

(i) simulating transdermal alcohol concentration data while accounting for bodily variation in that TAC simulation,

(ii) generating TAC data for population size $P$,

(iii) computing the maximum likelihood estimator for the joint mixing distribution of the diffusivity parameters.

For this thesis research, several Matlab functions were written to carry out these three main tasks and any necessary subtasks. A wrapper program titled Run_NPAG_clone.m (recall NPAG is an acronym for Nonparametric Adaptive 
Grid) provides appropriate initialization values and runs functions in the appropriate order to complete items (i) through (iii). See Figure B.1 for the program flow diagram. The numbering of the diagram arrows provides the precise order of file execution.

Figure B.1: Matlab Program Flow Diagram

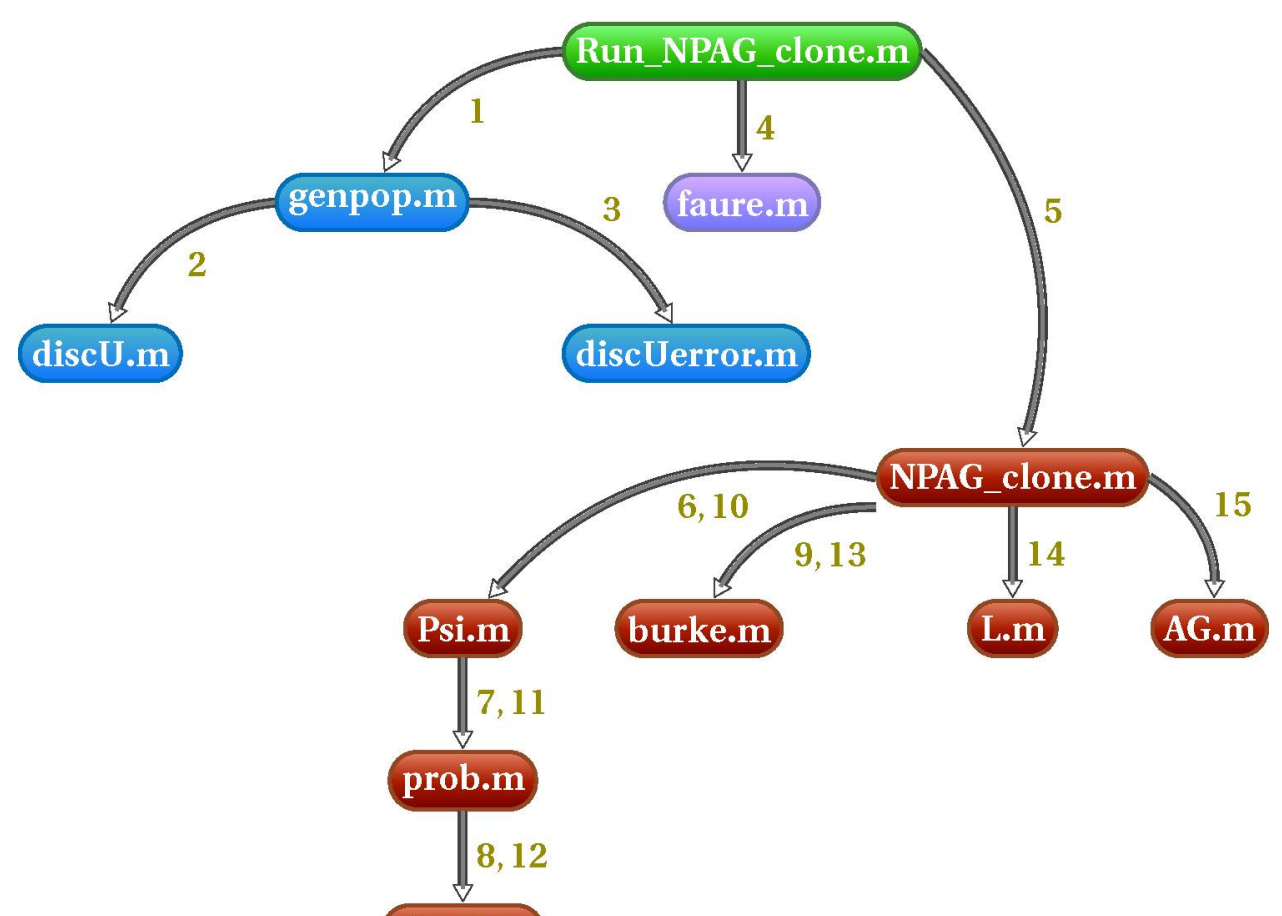

discU.m

To be clear, the Matlab code is not provided in this document, but the majority of the programs are transcribed into algorithms in this appendix as pseudocode, for your convenience, in the event that you do not wish to use 
or do not have access to the Matlab proprietary license. The Matlab functions do not necessarily perfectly align with the algorithms defined here since some subroutines are possibly redesigned to optimize Matlab efficiency. When specific Matlab code does directly align with an algorithm in this Appendix, the two will share names. For further detail, read the next paragraph.

The blue subroutines in the diagram implement item (i) via discUerror.m and item (ii) via genpop.m. The red subroutines in the diagram implement item (iii) via NPAG_clone.m and all of its supporting functions. The function L.m takes a vector and applies the log to each entry and then returns the sum of these values. The function AG.m executes the Adaptive Grid protocol to isolate the most probable support points for the joint mixing distribution. These two processes executed in L.m and AG.m are incorporated into the NPAG algorithm in Algorithm 4 so their respective algorithms are not provided separately as in the Matlab wrapper. The lavender subroutine in the diagram is the faure.m Matlab function, originally a FORTRAN method, that generates the initial grid used in the Adaptive Grid protocol via a quasirandom sequence (see YouTube video by Dr. Hayes [7]); which is tested to be extremely efficient in optimization algorithms. I am not aware of publicly available algorithm for this function. However, other grid initializations are also completely viable. 
Please note that burke.m, developed by James Burke, will not be provided in this paper, but a thorough description is contained in the article titled, "An algorithm for nonparametric estimation of a multivariate mixing distribution with applications to population pharmacokinetics" [25]. The burke.m executes an implementation of the primal-dual interior-point (PDIP) algorithm appropriate for this specific problem.

Algorithms 1, 2, and 3 were orignally developed by Dr. Melike Sirlanci [23] for her doctoral dissertation. Algorithms 4 through 8 were developed by a research group and are outlined in the aforementioned research paper [25]. These eight algorithms were all modified to some degree in order to interface with the goals of this research. 


\section{B.1 Variables, Constants, and Parameters}

The algorithms in the following sections utilize certain variables, constants, and parameters. In an effort for each algorithm to display definiteness, this section gives a brief overview of how each of these is defined, implemented, and provides notes, when necessary, to enlighten the reader to required characteristics or connections to certain algorithms. In the following sections, the name of any algorithm defined in this appendix will be highlighted in red for easy identification.

\begin{tabular}{|c|l|}
\hline $\mathbf{P}$ & The number of individuals in the experiment population. \\
\hline $\mathbf{N}$ & $\begin{array}{l}\text { The number of partitions of the skin depth. Note: There are } N+1 \\
\text { levels of skin depth. }\end{array}$ \\
\hline$\eta$ & The desired level of skin depth. \\
\hline Tint & $\begin{array}{l}\text { The total interval of time for the drinking episode. Note: All drinking } \\
\text { episodes are assumed to be identically long. }\end{array}$ \\
\hline$\tau$ & $\begin{array}{l}\text { The uniform length of a sub-interval of time. Note: } \tau \text { must evenly } \\
\text { divide Tint. }\end{array}$ \\
\hline $\mathbf{T}$ & $\begin{array}{l}\text { The number of partitions of the time interval Tint given by } \frac{\operatorname{Tint}}{\tau} . \\
\text { Note: There are } T+1 \text { points of time. }\end{array}$ \\
\hline
\end{tabular}




\begin{tabular}{|c|c|}
\hline $\mathbf{q}=\left\langle\mathbf{q}_{1}, \mathbf{q}_{2}\right\rangle$ & The vector of the parameters of diffusivity. \\
\hline$\alpha(\mathbf{t})$ & $\begin{array}{l}\text { The alcohol input into the system at the bottom }(\eta=1) \text { of the } \\
\text { dermal layer. It is currently being directly generated in the } \\
\text { discU algorithm. Note 1: For simplicity, every person in the } \\
\text { population is assumed to have the same drinking episode. Note } \\
\text { 2: The discU algorithm could easily be adjusted so that this } \\
\text { function could be passed directly. }\end{array}$ \\
\hline$\hat{\mathbf{c}}(\eta)$ & $\begin{array}{l}\text { A vector function which interpolates rows which correspond to } \\
\text { a specific } \eta \text { level of the dermal layer of the } U^{N, T} \text { matrix. (Note: } \\
\text { The choice of } N \text { determines the available } \frac{i}{N} \text { levels.) }\end{array}$ \\
\hline $\mathbf{q}_{\mu}$ & $\begin{array}{l}\text { The vector of mean values of the normal distribution for } q= \\
\left\langle q_{1}, q_{2}\right\rangle \text {. }\end{array}$ \\
\hline $\mathbf{q}_{\sigma}$ & $\begin{array}{l}\text { The vector of standard deviation values of the normal distri- } \\
\text { bution for } q=\left\langle q_{1}, q_{2}\right\rangle \text {. Note: This should be sufficiently small } \\
\text { since the dermal tissue of the majority of members in the pop- } \\
\text { ulation is assumed to exhibit low biological variation. }\end{array}$ \\
\hline $\mathbf{y}$ & $\begin{array}{l}\text { The } 1 \times(T+1) \text { vector of alcohol concentration data at time } t_{0}=0 \\
\text { to } t_{T}=T \tau \text { for a given } \eta \text { depth from discU. }\end{array}$ \\
\hline
\end{tabular}




\begin{tabular}{|c|c|}
\hline$\sigma$ & $\begin{array}{l}\text { The standard deviation of the normally distributed biological error in } \\
\text { discUerror. }\end{array}$ \\
\hline$\epsilon$ & $\begin{array}{l}\text { The biological error vector associated with an individual at each dis- } \\
\text { cretized point of time at } j \tau \text { for } j=0, \ldots, T \text {. }\end{array}$ \\
\hline $\mathbf{y}_{\epsilon}$ & $\begin{array}{l}\text { The } 1 \times(T+1) \text { vector of alcohol concentration data at time } t_{0}=0 \text { to } \\
t_{T}=T \tau \text { for a given } \eta \text { depth from disc } U \text { which also accounts for bio- } \\
\text { logical error } \epsilon \text {. Note: This is for one individual. }\end{array}$ \\
\hline $\mathbf{Y}_{e}$ & $\begin{array}{l}\text { The } P \times(T+1) \text { matrix of alcohol concentration values for a size } P \text { pop- } \\
\text { ulation. Note: Each row correlates to an individual's measurements } \\
\text { simulated for a fixed } \eta \text { depth input over time points } t_{0}=0 \text { to } t_{T}=T \tau \text {. }\end{array}$ \\
\hline $\mathbf{U}^{\mathbf{N}, \mathbf{T}}$ & $\begin{array}{l}\text { The matrix of alcohol concentration values discretizing the } x(t, \eta ; q) \\
\text { function with } N+1 \text { rows corresponding to each } \frac{i}{N} \text { depth for } i= \\
0, \ldots, N \text { and } T+1 \text { columns corresponding to each } j \tau \text { time interval for } \\
j=0, \ldots, T \text {. }\end{array}$ \\
\hline$\Omega$ & The parameter space for the support of $q=\left\langle q_{1}, q_{2}\right\rangle$. \\
\hline $\mathbf{f}_{q_{1}}$ & The probability density function of $q_{1}$. \\
\hline $\mathbf{f}_{\mathbf{q}_{2}}$ & The probability density function of $q_{2}$. \\
\hline
\end{tabular}




\begin{tabular}{|c|c|}
\hline $\mathbf{F}^{\mathrm{ML}}$ & $\begin{array}{l}\text { The maximum likelihood estimator for the joint mixing distribution } \\
\text { of the parameters of diffusivity. }\end{array}$ \\
\hline$\ell(\bullet)$ & The log-likelihood of $\bullet$. \\
\hline$\xi$ & The grid of support points (as a vector) for $F^{M L}$. \\
\hline$\xi^{0}$ & The initial grid of support points (as a vector) for $F^{M L}$. \\
\hline$\xi^{\mathbf{c}}$ & The condensed grid of support points (as a vector) for $F^{M L}$. \\
\hline$\xi \mathbf{e}$ & The expanded grid of support points (as a vector) for $F^{M L}$. \\
\hline $\mathbf{w}$ & $\begin{array}{l}\text { The vector of weights of each support point in the grid of support } \\
\text { points for } F^{M L} \text {. Note: } \sum_{k=1}^{K} w_{k}=1 \text { and } w_{k} \text { is proportional to } \mathbb{P}\left(q=\xi_{k}\right) \text {. }\end{array}$ \\
\hline $\mathbf{a}$ & $\begin{array}{l}\text { The lower bounds of possible values of support points in the } q_{1} \text { and } \\
q_{2} \text { directions of the grid of support points. }\end{array}$ \\
\hline $\mathbf{b}$ & $\begin{array}{l}\text { The upper bounds of possible values of support points in the } q_{1} \text { and } \\
q_{2} \text { directions of the grid of support points. }\end{array}$ \\
\hline $\operatorname{eps}, \Delta_{\mathbf{G}}$ & The accuracy variable of the NPAG algorithm. \\
\hline$\Delta_{\mathbf{e}}$ & The upper bound of the allowable value of the accuracy variable eps. \\
\hline$\Delta_{\mathrm{D}}$ & $\begin{array}{l}\text { The lower bound of the allowable distance between any two grid sup- } \\
\text { port points. }\end{array}$ \\
\hline
\end{tabular}




\begin{tabular}{|c|c|}
\hline$\Delta_{\mathbf{L}}$ & $\begin{array}{l}\text { The upper bound of the allowable difference between two iteration } \\
\text { of log-likelihood calculations. This is utilized to check if the log- } \\
\text { likelihood between two iterations has reduced to be less than this } \\
\text { value. This is one criterion for stopping the algorithm after the grid of } \\
\text { support is condensed. }\end{array}$ \\
\hline$\Delta_{\mathbf{F}}$ & $\begin{array}{l}\text { The upper bound of the allowable difference between two iterations } \\
\text { of log-likelihood calculations. This is one of the criterion used to de- } \\
\text { termine whether the NPAG will terminate or not. The main algorithm } \\
\text { loop will continue as long as the difference of log-likelihood values is } \\
\text { larger than } \Delta_{F} \text { OR the accuracy variable eps value is greater than } \Delta_{e} \text {. }\end{array}$ \\
\hline$\Delta_{\mathbf{w}}$ & The lower bound of the probabilities of the weights $w$. \\
\hline MAXCYCLES & $\begin{array}{l}\text { The upper bound of the number of allowable iterations of the NPAG } \\
\text { algorithm. Note that this should be set to a high positive integer value } \\
\text { to ensure convergence to the local maximum likelihood. This should } \\
\text { only function as a fail-safe from an untenable run-time. }\end{array}$ \\
\hline
\end{tabular}




\section{B.2 Data Simulation Algorithms}

\section{B.2.1 Algorithm 1: genpop}

The simulated TAC data for a population of $P$ subjects is needed for the MLE NPAG algorithm. The following algorithm generates the data vector for $P$ subjects and outputs a $P \times(T+1)$ matrix of values. This algorithm is called in the wrapper program Run_NPAG_clone.

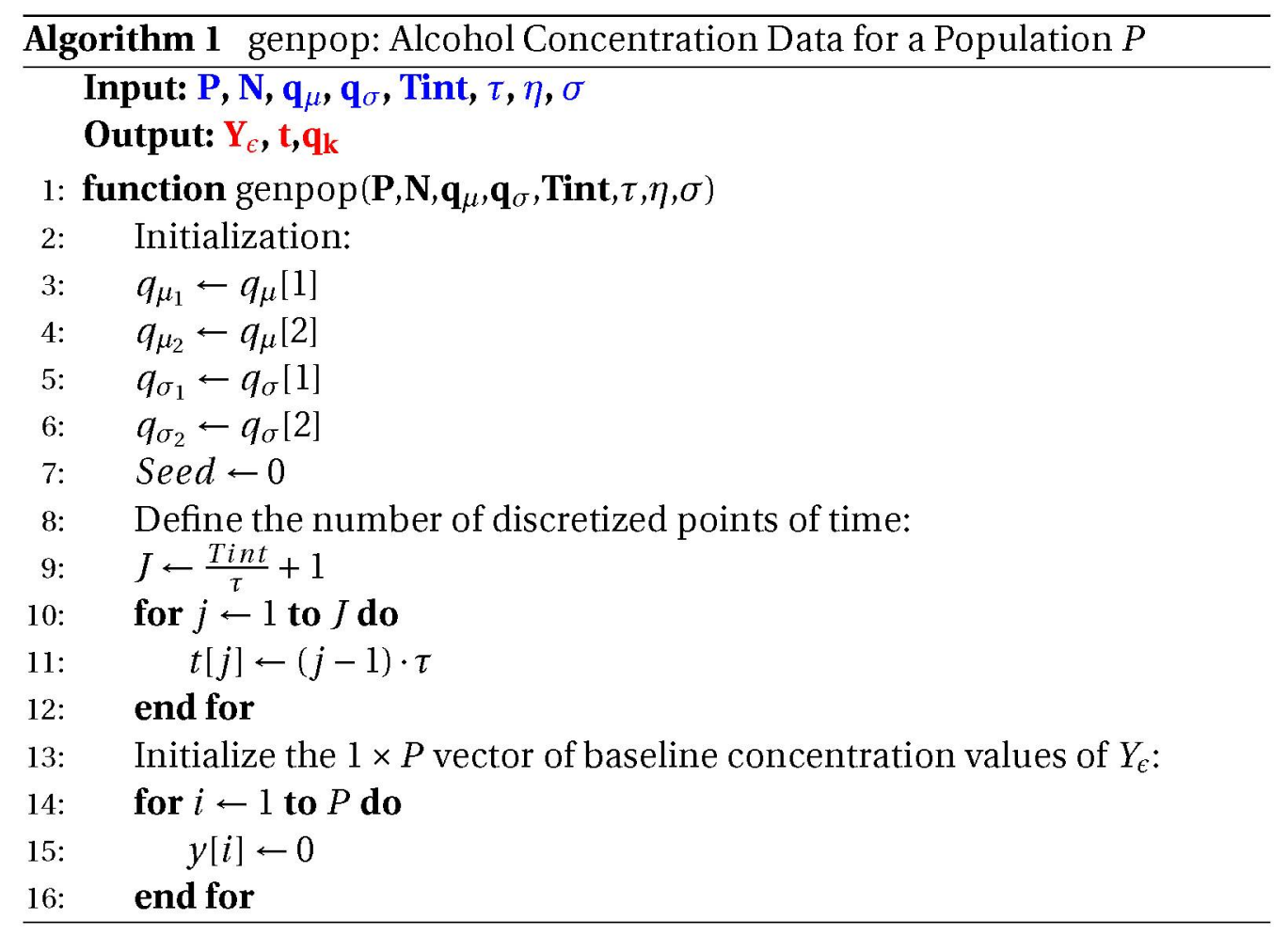




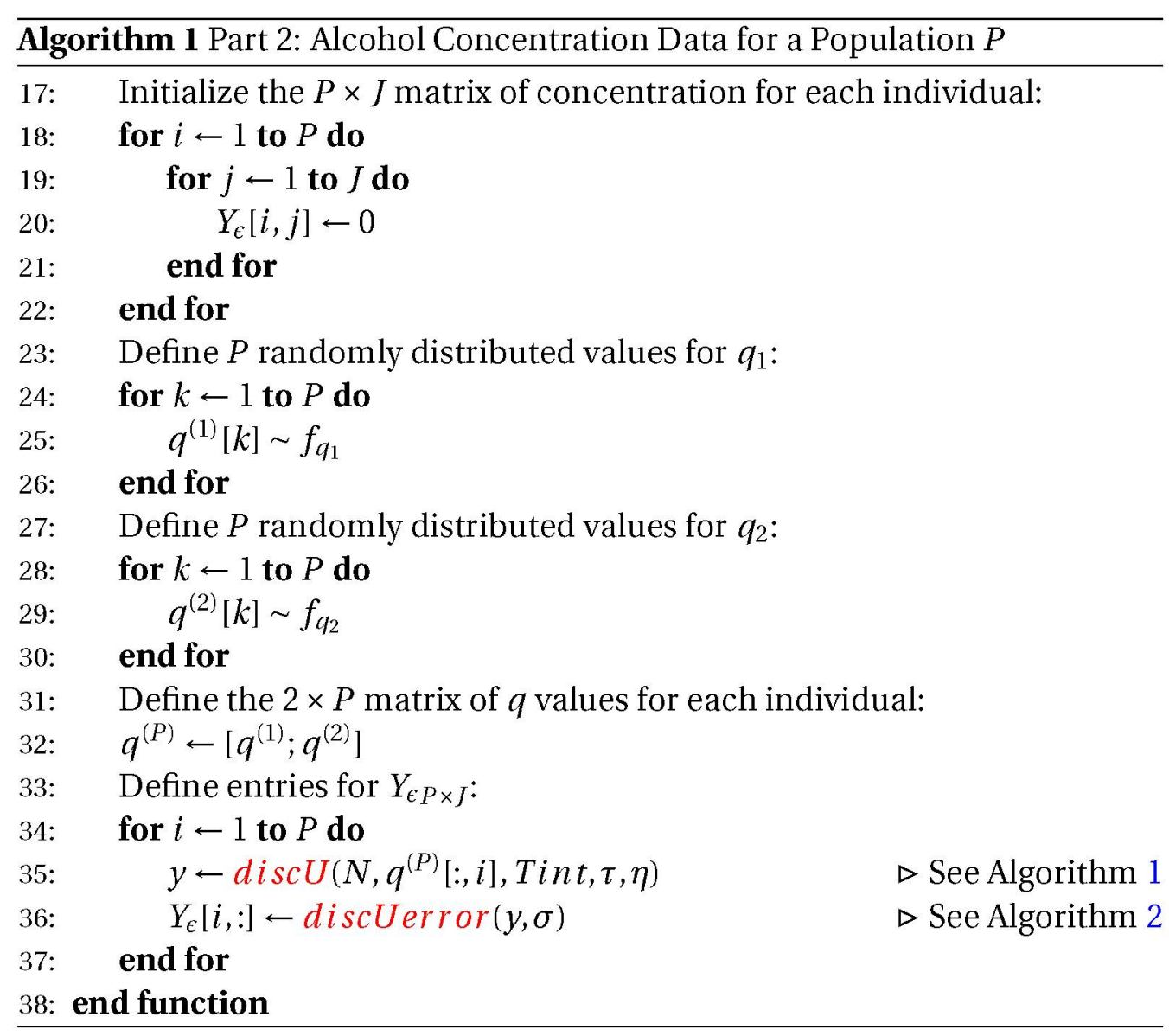




\section{B.2.2 Algorithm 2: discU}

The algorithm in this subsection details the process of discretizing the heat equation with only one spacial depth dimension via the finite element method. This version of the algorithm supposes two parameters of diffusivity for the proportional relationships of how alcohol diffuses across dermal tissue boundaries. Chapter 2 carefully explains the development of each step below. This algorithm is called by the genpop and prob algorithms in Algorithm 1 and Algorithm 6 respectively. It is also called indirectly by discUerror in Algorithm 3 since that algorithm is directly reliant on the data generated by discU.

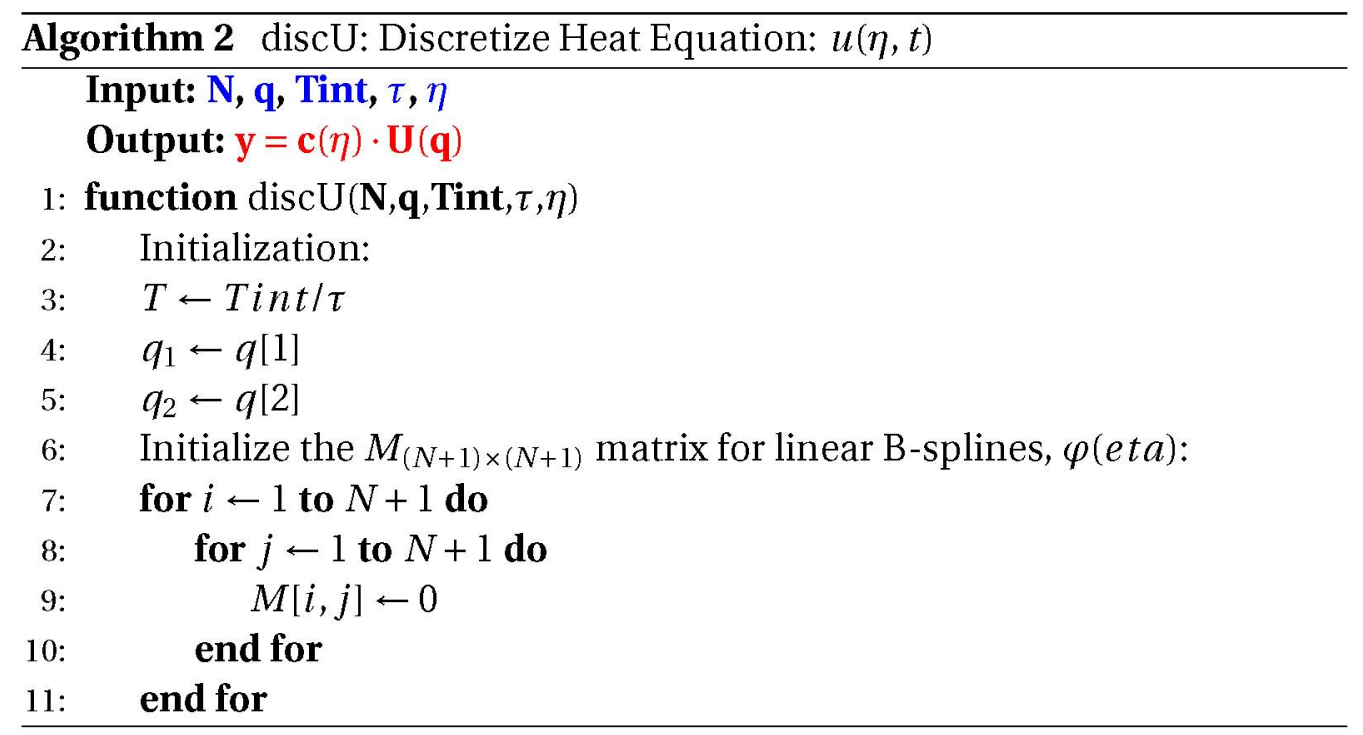




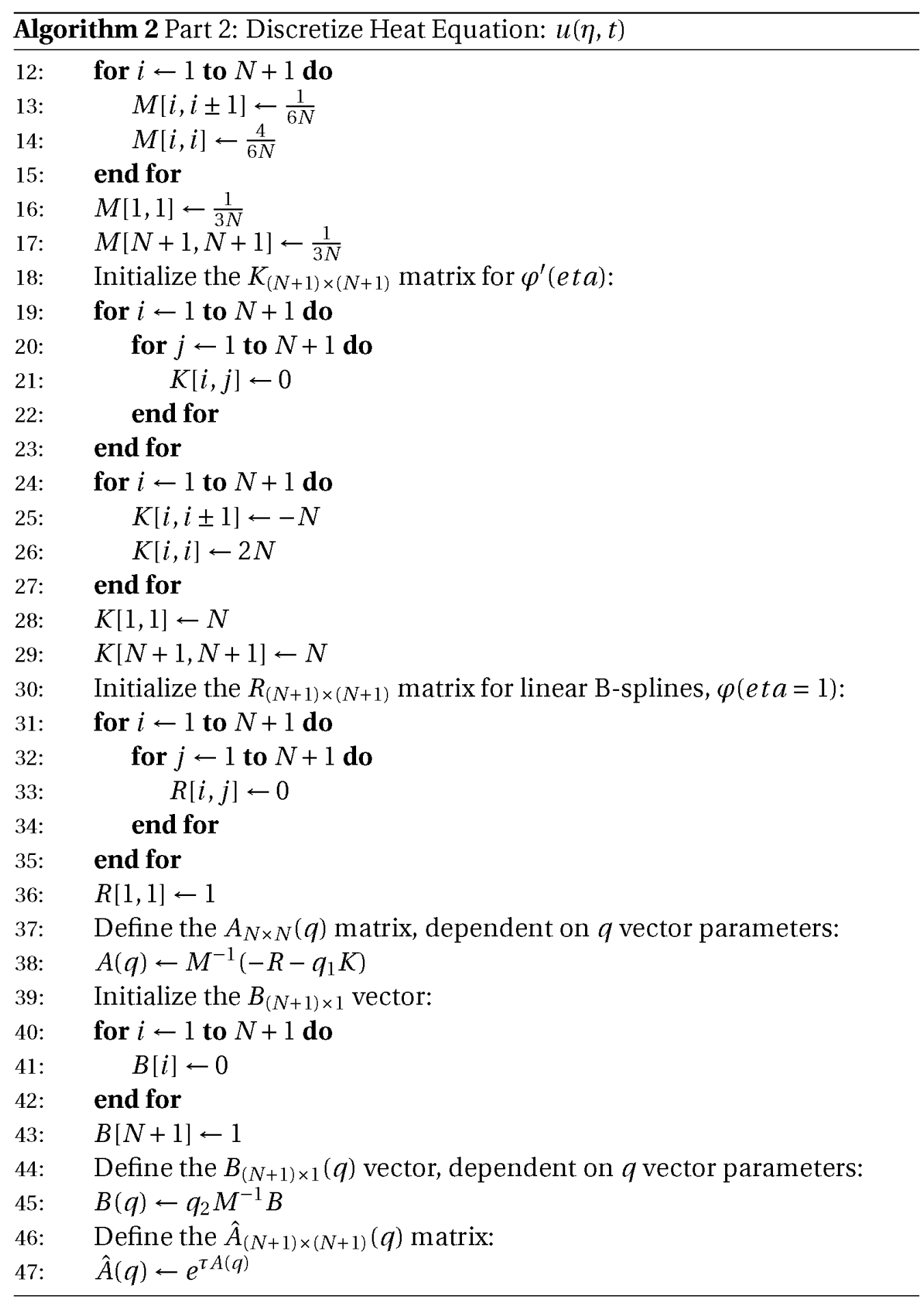




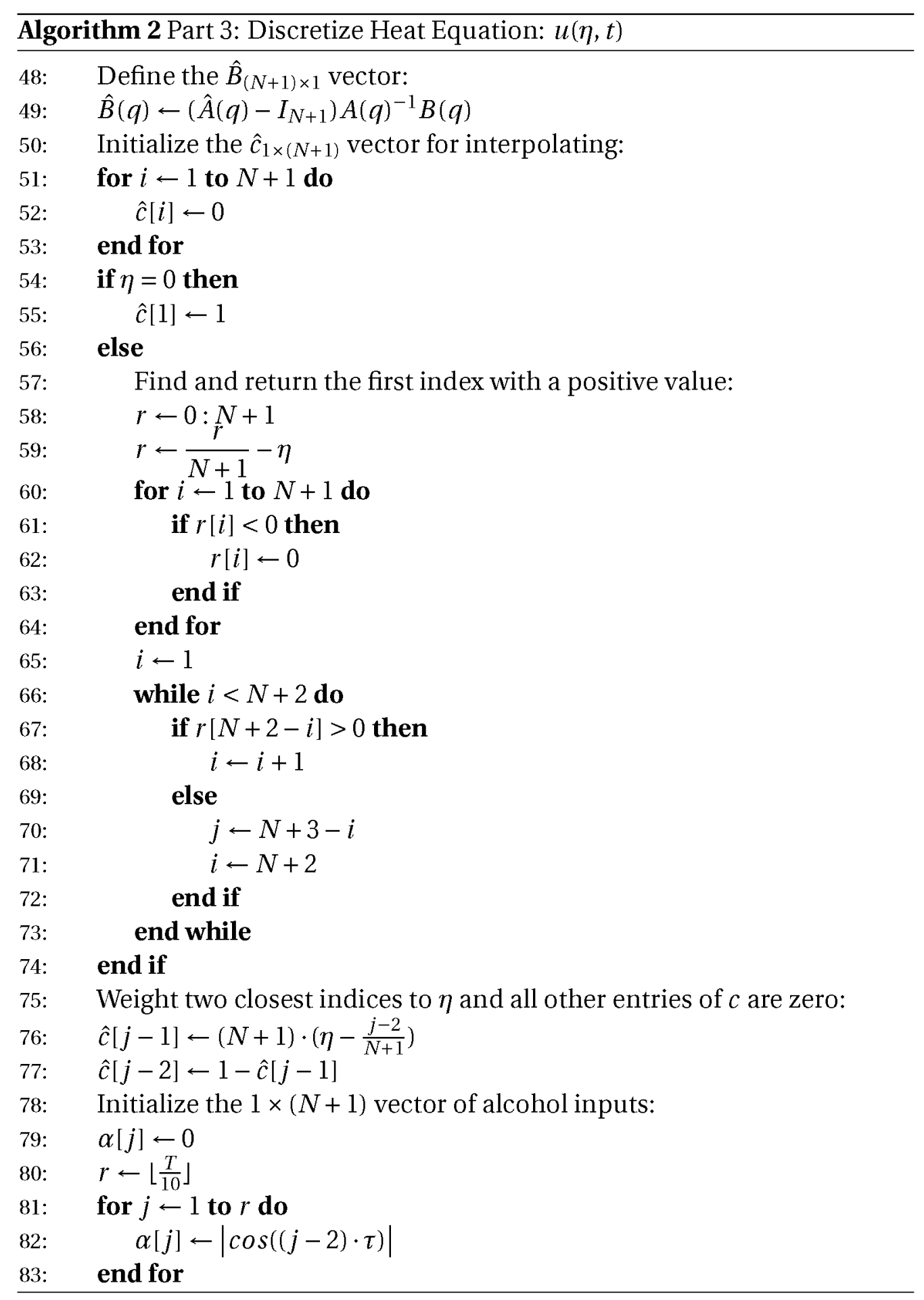




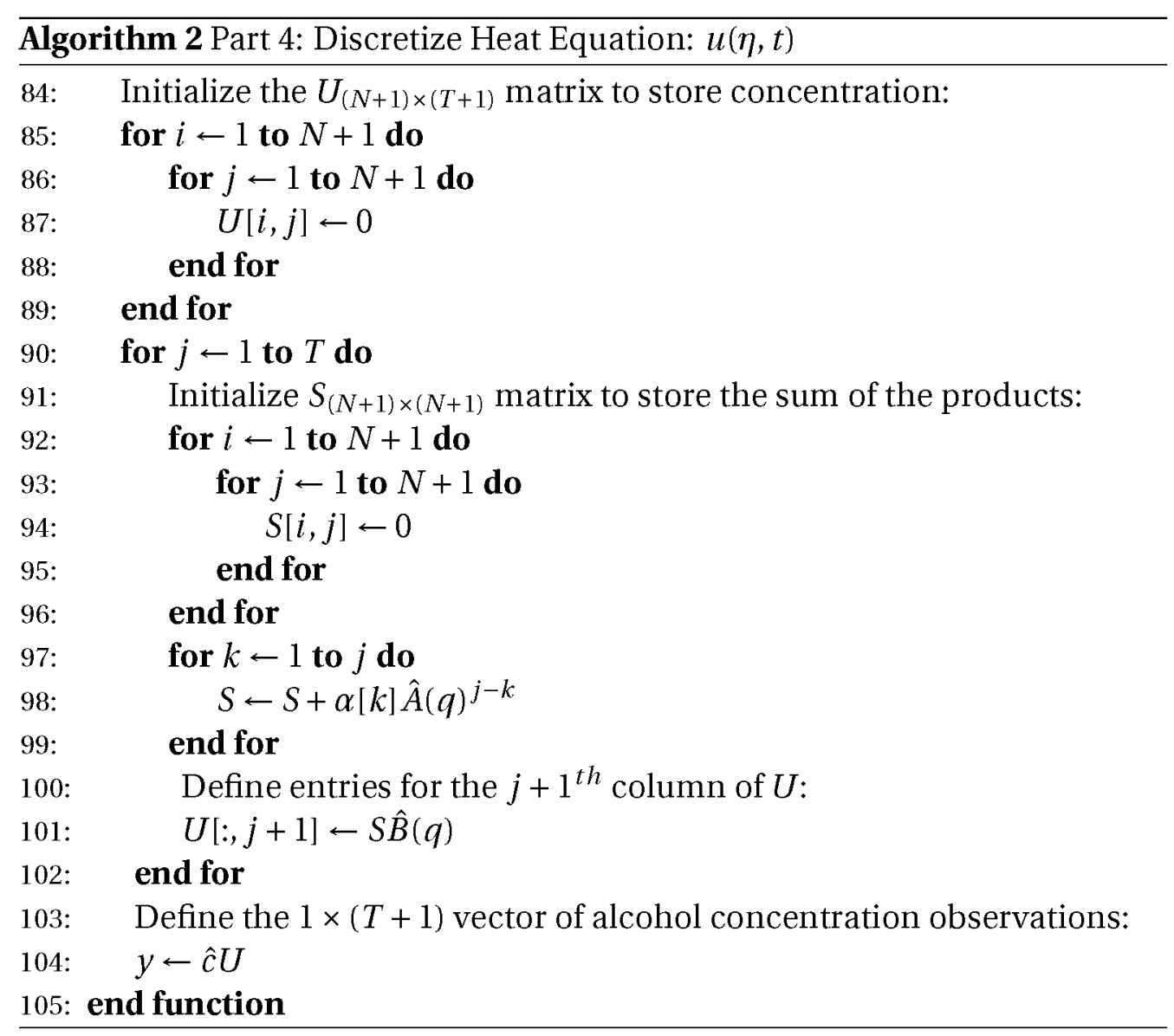




\section{B.2.3 Algorithm 3: discUerror}

This algorithm takes the discretized heat equation data from the discU algorithm (see Algorithm 2) which is calculated at a specific $\eta$ depth and adds an appropriate error sampled from a normal distribution with mean 0 and standard deviation $\sigma$. This is an attempt accurately account for biological variation in the dermal layer of an individual. This algorithm is called by the genpop algorithm in Algorithm 1.

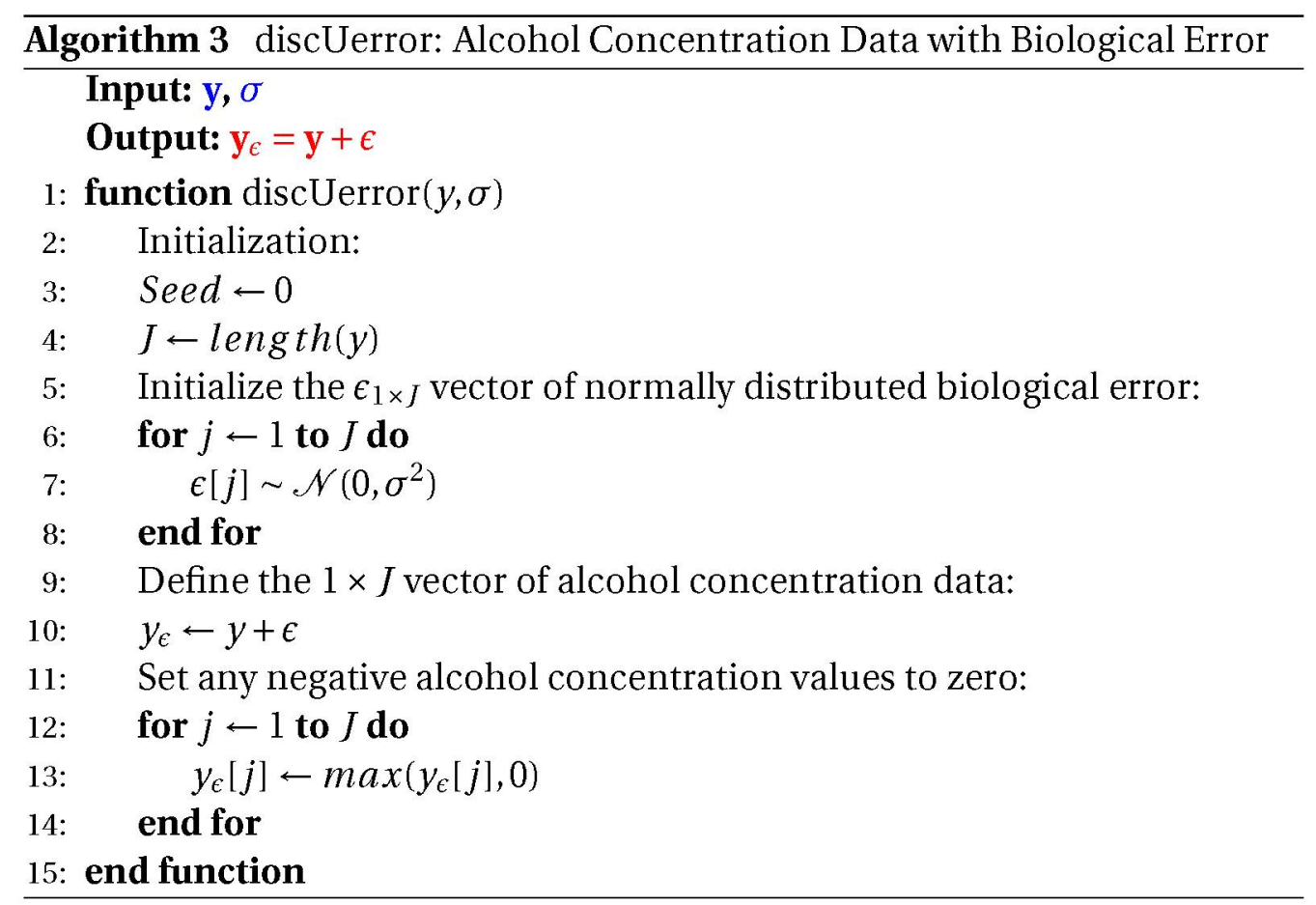




\section{B.3 Maximum Likelihood Estimator Algorithms}

\section{B.3.1 Algorithm 4: Nonparametric Adaptive Grid Algorithm}

This algorithm inputs population data, simulated or experimental, and calculates the maximum likelihood estimator for the joint mixing distribution of the parameters of diffusivity. Helper algorithms are defined in the following subsections. Please see the paper titled "An algorithm for nonparametric estimation of a multivariate mixing distribution with applications to population pharmacokinetics" [25] for a more complete explanation.

The acronym PDIP means primal-dual interior-point method. Essentially, this is the burke algorithm, developed by James Burke, which will not be provided here, but a thorough description is provided in the aforementioned paper. This algorithm is called in the wrapper program Run_NPAG_clone. 


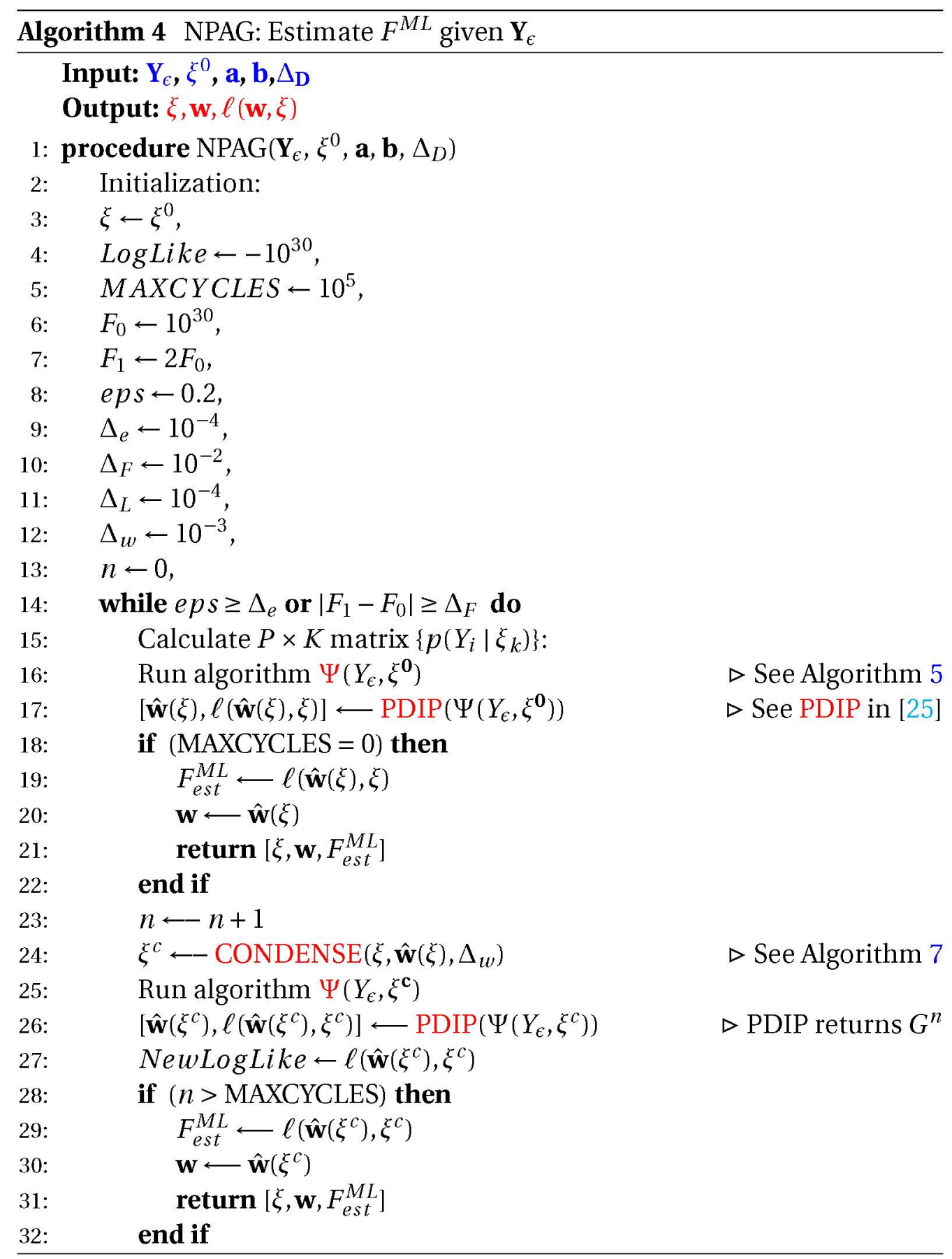




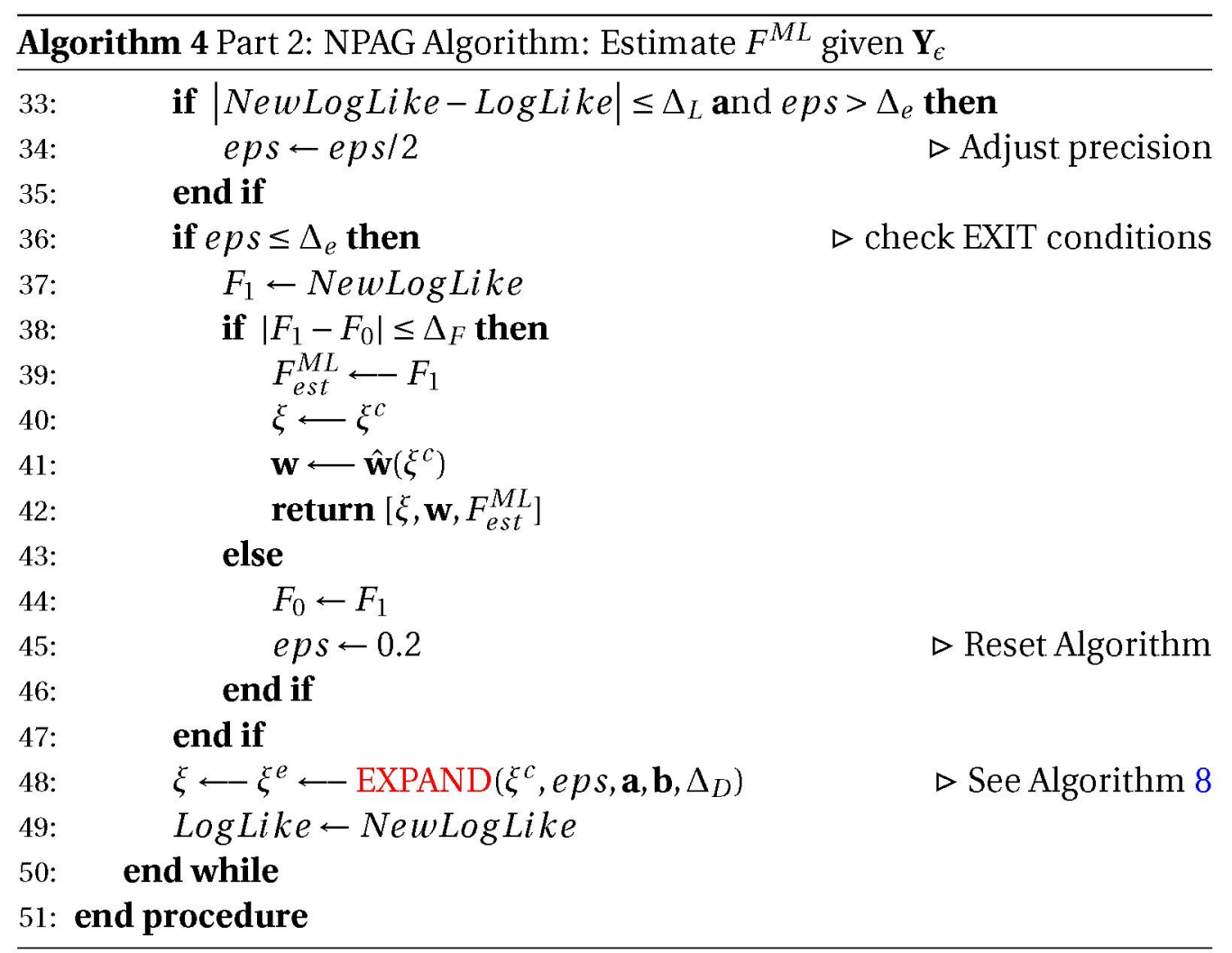




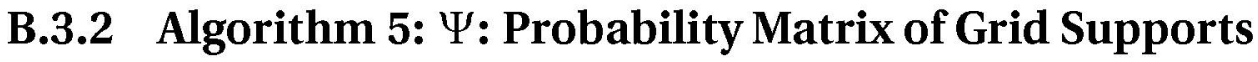

The algorithm in this subsection completes a vital subtask of the NPAG algorithm. It accepts a grid of support points and data observations and calculates the probability of each $Y_{i}$ observation given each support point; in notation, $p\left(Y_{i} \mid \xi_{j}\right)$. This algorithm is called by the NPAG algorithm in Algorithm 4 .

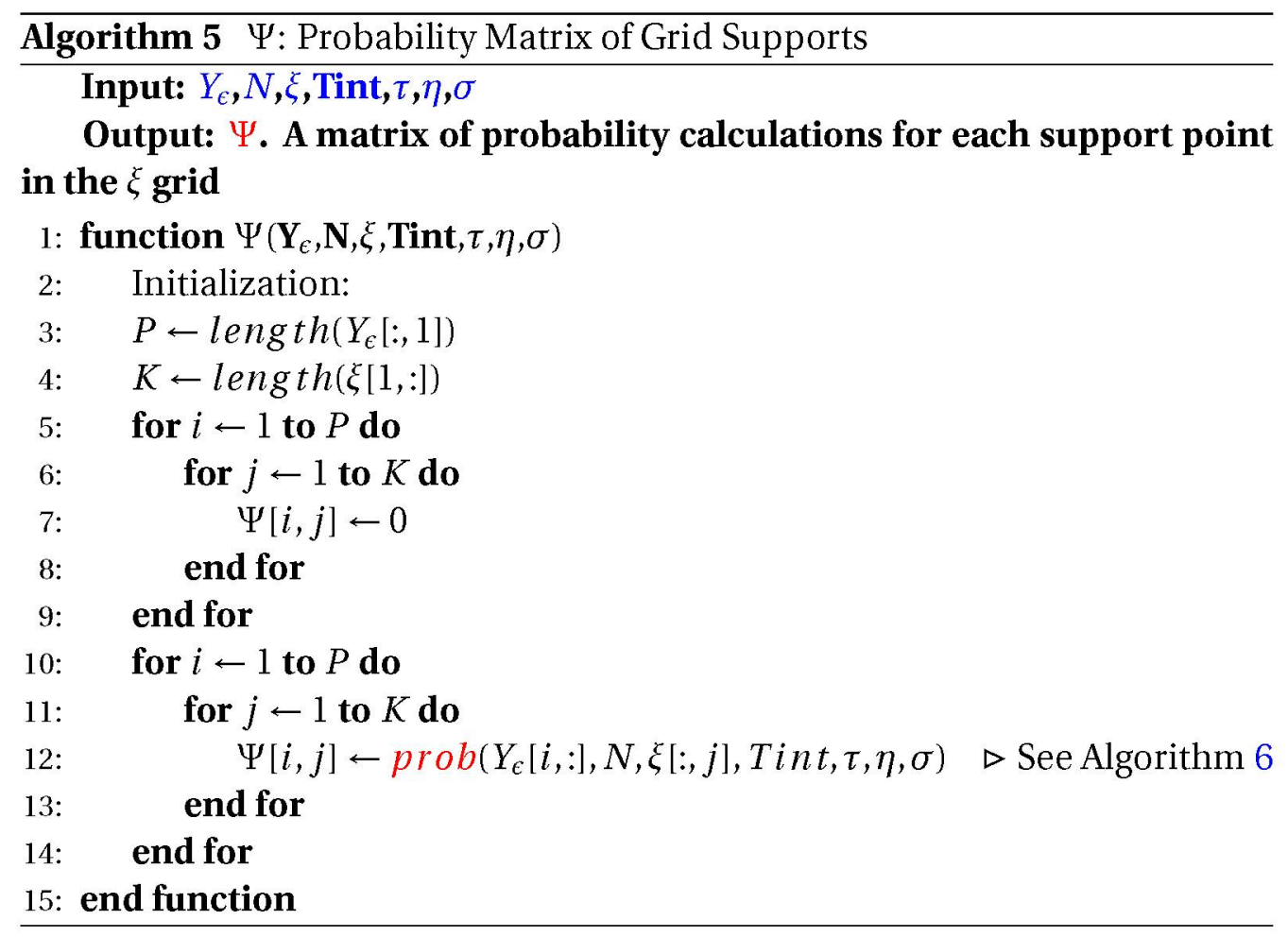




\section{B.3.3 Algorithm 6: Probability of $Y_{i}$ Given the $\xi_{j}$ Support}

\section{Point}

This algorithm calculates the probability of a $Y_{i}$ observation given a support point; in notation, $p\left(Y_{i} \mid \xi_{j}\right)$. This algorithm is called by the $\Psi$ algorithm in Algorithm 5.

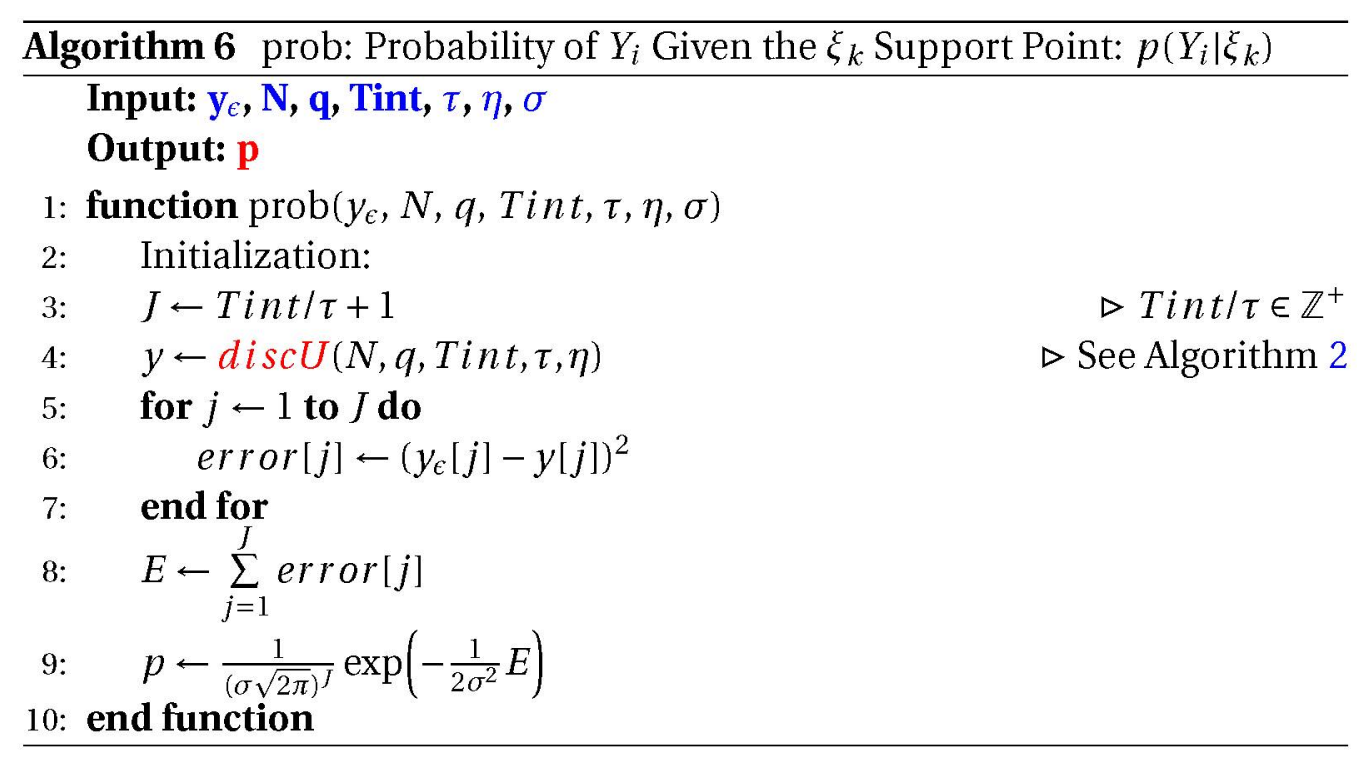




\section{B.3.4 Algorithm 7: Condense Grid of Support Points}

This subsection describes the Condense Grid protocol of the Adaptive grid portion of the NPAG algorithm in Algorithm 4. Essentially, this removes all support points from the possible grid of support points when a given support point has sufficiently low probability.

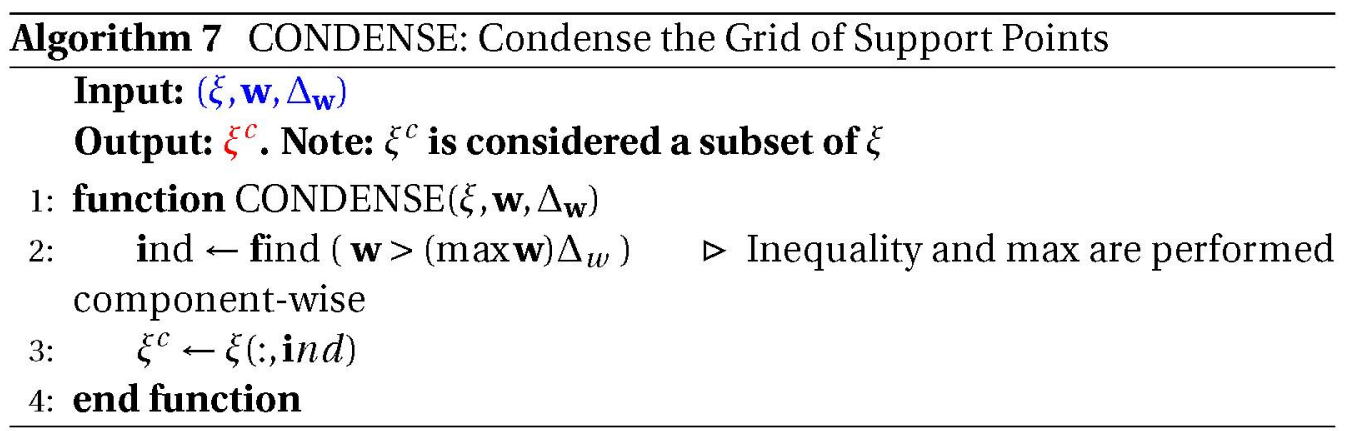




\section{B.3.5 Algorithm 8: Expand Grid of Support Points}

This subsection describes the Expand Grid protocol of the Adaptive grid portion of the NPAG algorithm in Algorithm 4. Essentially, this adds additional support points within a prescribed distance of the current grid of condensed support points (see Algorithm 7). This occurs after the likelihood difference has been calculated for the entire condensed grid of support points, but the absolute difference between two iterations of the log-likelihood does not shrink to a sufficiently small value $\Delta_{L}$.

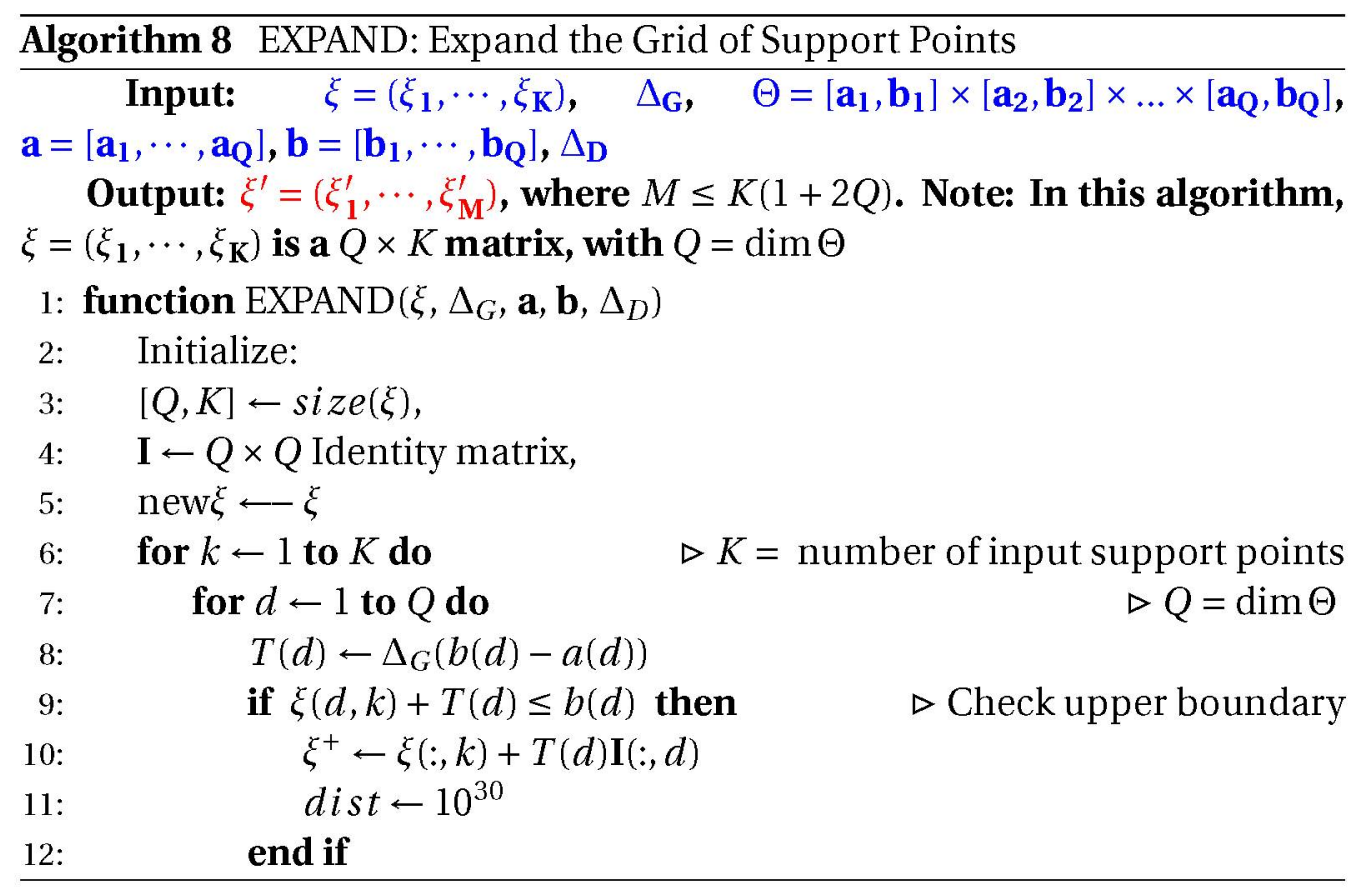




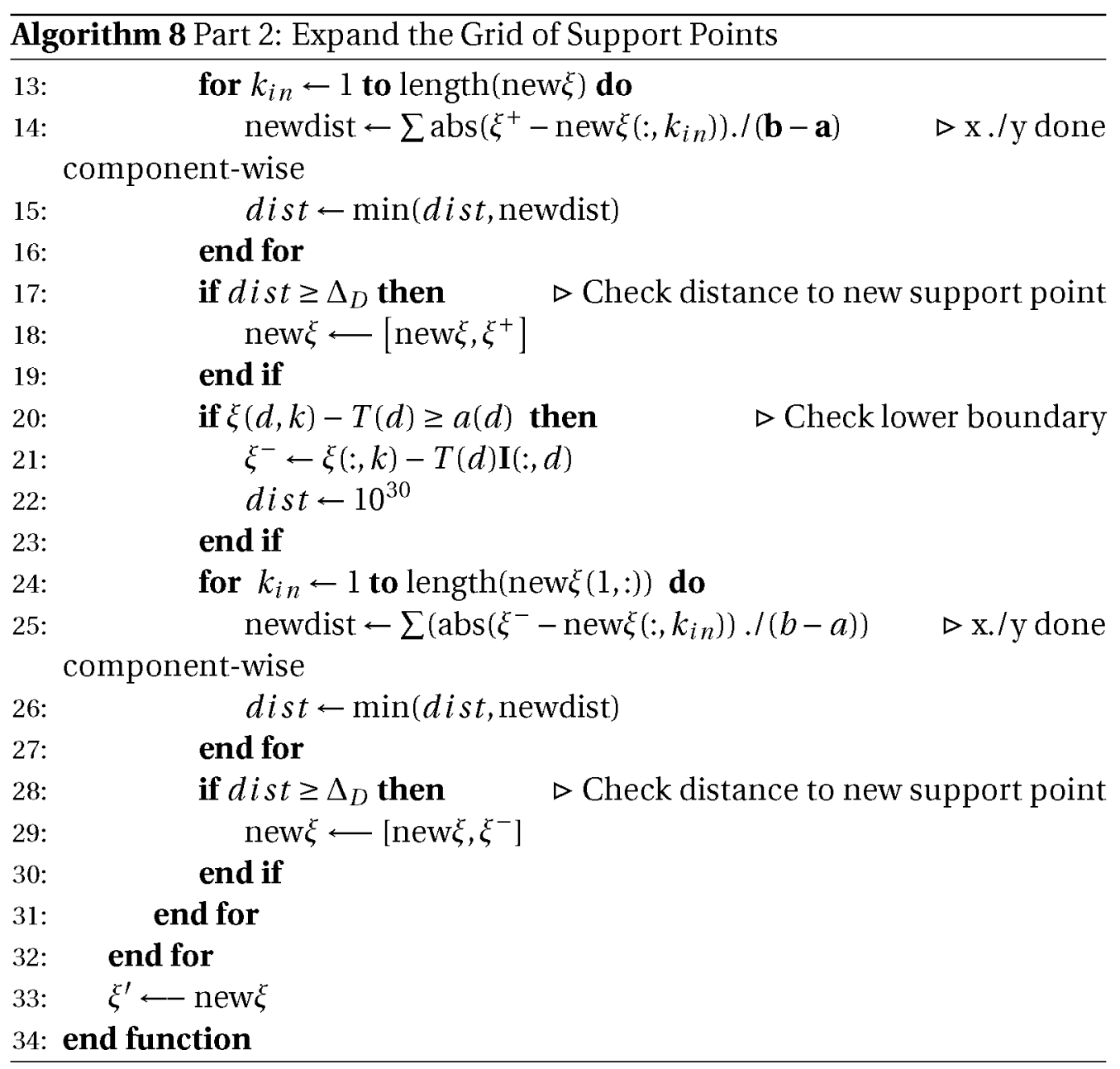




\section{LIST OF FIGURES}

1.1 SCRAM CAM® Alcohol Biosensor Ankle Device $\ldots . . \ldots . . . .8$

1.2 WrisTAS7® Alcohol Biosensor Wrist Device . . . . . . . . . . . 9

1.3 BACtrack Skyn Alcohol Biosensor Wrist Device . . . . . . . . . . . 11

1.4 Thesis Overview Diagram . . . . . . . . . . . . . . . . . . . . . . 13

2.1 Alcohol Diffusing Through the Dermal Layer . . . . . . . . . . 17

2.2 Heat Transfer Through a Rod . . . . . . . . . . . . . . . . . 18

2.3 Dermal Layer Orientation With Respect to an Axis . . . . . . . . 23

2.4 Example of B-Spline Curve-Fitting $\ldots \ldots \ldots \ldots \ldots$

2.5 Example of linear B-Splines, $N=5 \ldots \ldots \ldots \ldots$

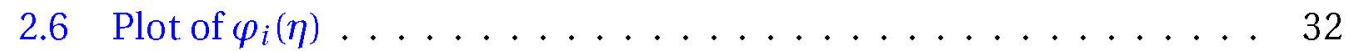

2.7 Plot of $\varphi_{i}(\eta)$ and $\varphi_{i+1}(\eta) \ldots \ldots \ldots \ldots \ldots$

2.8 Plot of $\varphi_{0}(\eta) \ldots \ldots \ldots \ldots \ldots \ldots \ldots \ldots \ldots$

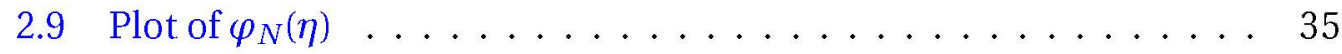

2.10 Example of partials of linear B-Splines, $N=5 \ldots \ldots \ldots$ 


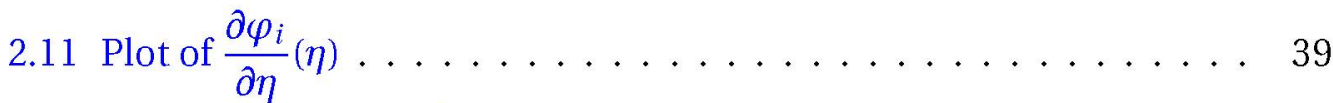

2.12 Plot of $\frac{\partial \varphi_{i}}{\partial \eta}(\eta)$ and $\frac{\partial \varphi_{i+1}}{\partial \eta}(\eta) \ldots \ldots \ldots \ldots \ldots \ldots$

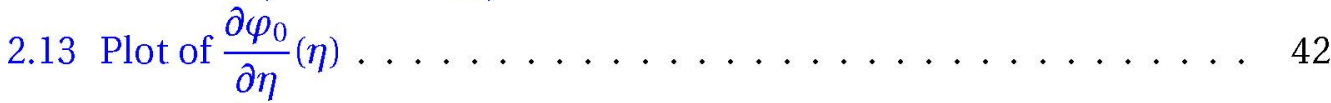

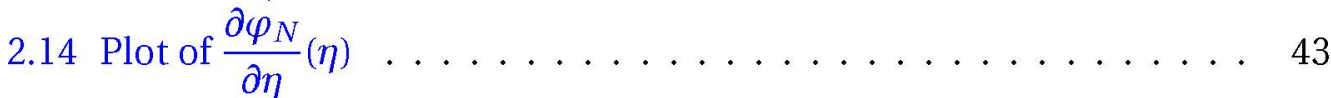

2.15 Example of order one B-Splines, $T=5 \ldots \ldots \ldots \ldots$

3.1 Convex Combination Example . . . . . . . . . . . . 67

4.1 Plot of Simulated TAC given $q_{1} \sim \mathcal{N}\left(1, \sigma^{2}\right)$ and $q_{2} \sim \mathscr{N}\left(1, \sigma^{2}\right) \ldots .76$

4.2 Plot of Simulated TAC given $q_{1} \sim 0.7 \mathscr{N}\left(0.7, \sigma^{2}\right)+0.3 \mathscr{N}\left(1, \sigma^{2}\right)$ and $q_{2} \sim \mathscr{N}\left(1, \sigma^{2}\right) \ldots \ldots \ldots \ldots \ldots \ldots \ldots$

4.3 Plot of Simulated TAC given $q_{1} \sim 0.7 \mathscr{N}\left(0.7, \sigma^{2}\right)+0.3 \mathscr{N}\left(1, \sigma^{2}\right)$ and $q_{2} \sim 0.3 \mathscr{N}\left(0.7, \sigma^{2}\right)+0.7 \mathscr{N}\left(1, \sigma^{2}\right) \ldots \ldots \ldots \ldots$

4.4 Plot of $D$-Function given $q_{1} \sim \mathcal{N}\left(1, \sigma^{2}\right)$ and $q_{2} \sim \mathcal{N}\left(1, \sigma^{2}\right) \ldots . \quad \ldots 7$

4.5 Plot of $D$-Function given $q_{1} \sim 0.7 \mathscr{N}\left(0.7, \sigma^{2}\right)+0.3 \mathscr{N}\left(1, \sigma^{2}\right)$ and $q_{2} \sim \mathscr{N}\left(1, \sigma^{2}\right) \ldots \ldots \ldots \ldots \ldots \ldots \ldots \ldots$

4.6 Plot of $D$-Function given $q_{1} \sim 0.7 \mathscr{N}\left(0.7, \sigma^{2}\right)+0.3 \mathscr{N}\left(1, \sigma^{2}\right)$ and $q_{2} \sim 0.3 \mathscr{N}\left(0.7, \sigma^{2}\right)+0.7 \mathscr{N}\left(1, \sigma^{2}\right) \ldots \ldots \ldots \ldots 9$

4.7 Comparison plot of the MLE and the Density Function $f_{q_{1}}$ given $q_{1} \sim \mathscr{N}\left(1, \sigma^{2}\right)$ and $q_{2} \sim \mathscr{N}\left(1, \sigma^{2}\right) \ldots \ldots \ldots 2$ 
4.8 Plot of the Density Function $f_{q_{1}}$ Overlaid with the MLE given $q_{1} \sim \mathscr{N}\left(1, \sigma^{2}\right)$ and $q_{2} \sim \mathscr{N}\left(1, \sigma^{2}\right) \ldots \ldots \ldots$

4.9 Comparison plot of the MLE and the Density Function $f_{q_{2}}$ given $q_{1} \sim \mathscr{N}\left(1, \sigma^{2}\right)$ and $q_{2} \sim \mathscr{N}\left(1, \sigma^{2}\right) \ldots \ldots \ldots 104$

4.10 Plot of the Density Function $f_{q_{2}}$ Overlaid with the MLE given $q_{1} \sim \mathscr{N}\left(1, \sigma^{2}\right)$ and $q_{2} \sim \mathscr{N}\left(1, \sigma^{2}\right) \ldots \ldots \ldots 105$

4.11 Comparison plot of the MLE and the Density Function $f_{q_{1}}$ given $q_{1} \sim 0.7 \mathscr{N}\left(0.7, \sigma^{2}\right)+0.3 \mathscr{N}\left(1, \sigma^{2}\right)$ and $q_{2} \sim \mathcal{N}\left(1, \sigma^{2}\right) \ldots \ldots 6$

4.12 Plot of the Density Function $f_{q_{1}}$ Overlaid with the MLE given $q_{1} \sim 0.7 \mathscr{N}\left(0.7, \sigma^{2}\right)+0.3 \mathscr{N}\left(1, \sigma^{2}\right)$ and $q_{2} \sim \mathcal{N}\left(1, \sigma^{2}\right) \ldots \ldots 7$

4.13 Comparison plot of the MLE and the Density Function $f_{q_{2}}$ given $q_{1} \sim 0.7 \mathscr{N}\left(0.7, \sigma^{2}\right)+0.3 \mathscr{N}\left(1, \sigma^{2}\right)$ and $q_{2} \sim \mathcal{N}\left(1, \sigma^{2}\right) \ldots \ldots 108$

4.14 Plot of the Density Function $f_{q_{2}}$ Overlaid with the MLE given $q_{1} \sim 0.7 \mathscr{N}\left(0.7, \sigma^{2}\right)+0.3 \mathscr{N}\left(1, \sigma^{2}\right)$ and $q_{2} \sim \mathcal{N}\left(1, \sigma^{2}\right) \ldots \ldots 109$

4.15 Comparison plot of the MLE and the Density Function $f_{q_{1}}$ given $q_{1} \sim 0.7 \mathscr{N}\left(0.7, \sigma^{2}\right)+0.3 \mathscr{N}\left(1, \sigma^{2}\right)$ and $q_{2} \sim 0.3 \mathscr{N}\left(0.7, \sigma^{2}\right)+0.7 \mathscr{N}\left(1, \sigma^{2}\right) 110$

4.16 Plot of the Density Function $f_{q_{1}}$ Overlaid with the MLE given $q_{1} \sim 0.7 \mathscr{N}\left(0.7, \sigma^{2}\right)+0.3 \mathscr{N}\left(1, \sigma^{2}\right)$ and $q_{2} \sim 0.3 \mathscr{N}\left(0.7, \sigma^{2}\right)+0.7 \mathscr{N}\left(1, \sigma^{2}\right) 111$ 
4.17 Comparison plot of the MLE and the Density Function $f_{q_{2}}$ given $q_{1} \sim 0.7 \mathscr{N}\left(0.7, \sigma^{2}\right)+0.3 \mathscr{N}\left(1, \sigma^{2}\right)$ and $q_{2} \sim 0.3 \mathscr{N}\left(0.7, \sigma^{2}\right)+0.7 \mathscr{N}\left(1, \sigma^{2}\right) 112$

4.18 Plot of the Density Function $f_{q_{2}}$ Overlaid with the MLE given $q_{1} \sim 0.7 \mathscr{N}\left(0.7, \sigma^{2}\right)+0.3 \mathscr{N}\left(1, \sigma^{2}\right)$ and $q_{2} \sim 0.3 \mathscr{N}\left(0.7, \sigma^{2}\right)+0.7 \mathscr{N}\left(1, \sigma^{2}\right) 113$

5.1 Bayesian Calibration for an Individual . . . . . . . . . . . . . 119

B.1 Matlab Program Flow Diagram . . . . . . . . . . . . . . . . . . . 158 


\section{LiST OF Algorithis}

1 genpop: Alcohol Concentration Data for a Population $P \quad \ldots \quad 166$

2 discU: Discretize Heat Equation: $u(\eta, t) \ldots \ldots \ldots \ldots$

3 discUerror: Alcohol Concentration Data with Biological Error . 172

$4 \quad$ NPAG: Estimate $F^{M L}$ given $\mathbf{Y}_{\epsilon} \ldots \ldots \ldots \ldots$

$5 \quad \Psi$ : Probability Matrix of Grid Supports . . . . . . . . . 176

6 prob: Probability of $Y_{i}$ Given the $\xi_{k}$ Support Point: $p\left(Y_{i} \mid \xi_{k}\right) \ldots 177$

7 CONDENSE: Condense the Grid of Support Points . . . . . . . 178

8 EXPAND: Expand the Grid of Support Points _ . . . . . . . . 179 


\section{BIBLIOGRAPHY}

[1] R. Berger and G. Casella, Statistical Inference, Cengage Learning, 2001.

[2] S. Boyd, Convex Optimization, Cambridge University Press, UK, Cambridge, 2009 .

[3] A. Chubatiuk, Nonparametric estimation of an unknown probability distribution using maximum likelihood and Bayesian approaches, dissertation, USC, 2013.

[4] V. Fedorov, Theory of Optimal Experiments, Academic Press, 1972.

[5] B. Fox, Algorithm 647: Implementation and relative efficiency of quasirandom sequence generators, ACM Trans. Math. Softw., 12 (1986), pp. 362376.

[6] J. HAWTHORNE AND M. WOJCIK, Transdermal alcohol measurement: A review of the literature, Canadian Society of Forensic Science Journal, 39 (2006), pp. 65-71. 
[7] B. HAYES, Youtube: Harvard Institute for Applied Computational Science, IACS Seminar: Orderly Randomness: Quasirandom Numbers and Quasi-Monte Carlo 02/06/15, 2015 (accessed online November 20, 2019). https://youtu.be/wGflWqY41RU.

[8] C. JoHnson, Numerical Solution of Partial Differential Equations by the Finite Element Method (Dover Books on Mathematics), Dover Publications, 2009.

[9] J. KiEFER AND J. WOLFOWITZ, Consistency of the maximum likelihood estimator in the presence of infinitely many incidental parameters, The Annals of Mathematical Statistics, 27 (1956), pp. 887-906.

[10] D. Kincaid AND W. ChenEY, Numerical Analysis: Mathematics of Scientific Computing, American Mathematical Society, 2002.

[11] T. Leffingwell, N. Cooney, J. Murphy, S. Luczak, G. Rosen, D. Dougherty, And N. BARnetT, Continuous objective monitoring of alcohol use: Twenty-first century measurement using transdermal sensors, ALCOHOLISM: CLINICAL AND EXPERIMENTAL RESEARCH, 37 (2013), pp. 16-22. 
[12] E. Lehmann AND G. Casella, Theory of Point Estimation, Springer, 1998.

[13] B. LINDSAY, The geometry of mixture likelihoods: A general theory, Annals of Statistics, 11 (1983), pp. 86-94.

[14] A. MALLET, A maximum likelihood estimation method for random coefficient regression models, Biometrika, 73 (1986), pp. 645-656.

[15] E. Nyman ANd A. Palmlov, The elimination of ethyl alcohol in sweat, Acta Physiologica, 74 (1936), pp. 155-159.

[16] P. Olver, Introduction to Partial Differential Equations, Springer, 2016.

[17] H. Prautzch, W. Boehm, and M. Paluszny, Bézier and B-Spline Techniques, Springer-Verlag, 2002.

[18] A. Roberts And D. VarberG, Convex functions, Academic Press, New York, New York, 1973.

[19] W. Rudin, Real and Complex Analysis, McGraw-Hill, Inc., New York, New York, 1987.

[20] — Functional Analysis, McGraw-Hill Book Co., 1991. 
[21] A. SCHUMITZKY, Nonparametric EM algorithms for estimating prior distributions, Applied Mathematics and Computation, 45 (1991), pp. 143157.

[22] W. Straus, Partial Differential Equations: An Introduction, John Wiley \& Sons, Ltd, Hoboken, New Jersey, 2007.

[23] M. Tuysuzoglu, Finite Dimensional Approximation and Convergence in the Estimation of the Distribution of, and Input to, Random Abstract Parabolic Systems with Application to the Deconvolution of Blood/Breath Alcohol Concentration from Biosensor Measured Transdermal Alcohol Level, dissertation, USC, 2018.

[24] S. Wright, Primal-Dual Interior-Point Methods, SIAM, Philadelphia, Pennsylvania, 1997.

[25] W. M. Yamada, M. Neely, J. Bartroff, D. Bayard, J. Burke, M. van Guilder, R. W. Jelliffe, A. Kryshchenko, R. Leary, T. Tatarinova, AND A. SCHUMITZKY, An algorithm for nonparametric estimation of a multivariate mixing distribution with applications to population pharmacokinetics, Pharmacokinetics, (2019), pp. 1-19. 


\section{Non-Exclusive Distribution License}

In order for California State University Channel Islands (CSUCI) to reproduce, translate and distribute your submission worldwide through the CSUCI Institutional Repository, your agreement to the following terms is necessary. The author(s) retain any copyright currently on the item as well as the ability to submit the item to publishers or other repositories.

By signing and submitting this license, you (the author(s) or copyright owner) grants to CSUCl the nonexclusive right to reproduce, translate (as defined below), and/or distribute your submission (including the abstract) worldwide in print and electronic format and in any medium, including but not limited to audio or video.

You agree that CSUCI may, without changing the content, translate the submission to any medium or format for the purpose of preservation.

You also agree that CSUCl may keep more than one copy of this submission for purposes of security, backup and preservation.

You represent that the submission is your original work, and that you have the right to grant the rights contained in this license. You also represent that your submission does not, to the best of your knowledge, infringe upon anyone's copyright. You also represent and warrant that the submission contains no libelous or other unlawful matter and makes no improper invasion of the privacy of any other person.

If the submission contains material for which you do not hold copyright, you represent that you have obtained the unrestricted permission of the copyright owner to grant CSUCI the rights required by this license, and that such third party owned material is clearly identified and acknowledged within the text or content of the submission. You take full responsibility to obtain permission to use any material that is not your own. This permission must be granted to you before you sign this form.

IF THE SUBMISSION IS BASED UPON WORK THAT HAS BEEN SPONSORED OR SUPPORTED BY AN AGENCY OR ORGANIZATION OTHER THAN CSUCI, YOU REPRESENT THAT YOU HAVE FULFILLED ANY RIGHT OF REVIEW OR OTHER OBLIGATIONS REQUIRED BY SUCH CONTRACT OR AGREEMENT.

The CSUCI Institutional Repository will clearly identify your name(s) as the author(s) or owner(s) of the submission, and will not make any alteration, other than as allowed by this license, to your submission.

Nonparametric estimation of blood alcohol concentration from transdermal alcohol measurements using alcohol biosensor devices

Title of Item

Nonparametric, Likelihood, Optimization, Biosensor, Alcohol

3 to 5 keywords or phrases to describe the item

Bryan E. Vader

Author(s) Name (Print)

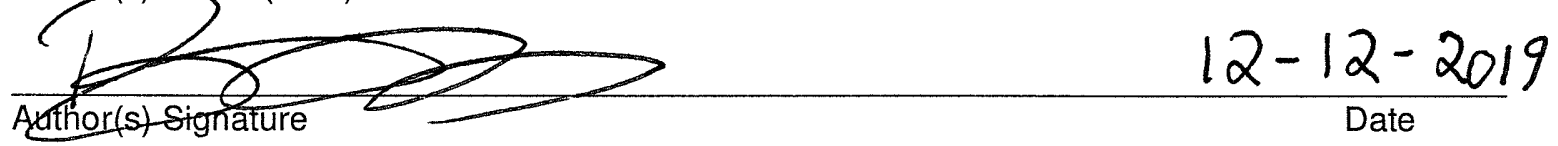

